THE FLORA OF THE TOWN OF SOUTHOLD, LONG ISLAND AND GARDINER'S ISLAND

By Stewart H. Burnham and Roy A. Latham

[Reprinted from TORREYA, Vol 14, NoS. I1.12, 1914.] 

[Reprinted from Tokreya, Vol. I4, No. II, Nov., I9I4.]

\title{
THE FLORA OF THE TOWN OF SOUTHOLD, LONG ISLAND AND GARDINER'S ISLAND
}

\author{
By Stewart H. Burnham and Roy A. Latham
}

The town of Southold is at the eastern end of the north shore of Long Island, extending westward for twenty miles through the villages of Orient, East Marion, Greenport, Southold, Peconic, Cutchogue to Mattituck and Laurel. The topography of the town affords a pleasing variation. On the north, paralleled with the coast of Long Island Sound, are rolling plains and hillocks; now forested, now barren, or broken by low-lying necks of sandy beaches and inlets. On the south, facing the bays and harbors, are extensive salt meadows drained by numerous tidal creeks: the marshes bordered, here and there, by a growth of cedar and hardwood. Between the waterways and tidal creeks sandy beaches extend far out: such areas often being covered with sprawling red cedars, pitch pine and beach plum shrubs. On one of these peninsulas at Orient over seventy-five species of lichens have been found, growing either on the low trees or on the ground: and on a single prostrate cedar fourteen species were found. Fertile, low, level farms lie principally through the center of the town of Southold. Among the more common plants found throughout the town but not known to occur at Orient, are: Skunk-cabbage, beech, witch-hazel, trailing arbutus and mountain laurel. These plants are found at Greenport, six miles west of Orient: and the local flora of these two places seem strangely different.

Gardiner's Island is situated ten miles southeast of Orient and three miles north of the Hampton shore. The flora is quite similar to that found about Orient; excepting the abundance of 201 
seaside knotweed, sea purslane, sea poppy, New England aster and Scotch thistle. The island is still wooded with the magnificent first-growth oak and beech forests: interposed with high rolling plains and rich luxuriant lowlands, side by side.

Roy A. Latham of Orient, from May 25, 1909, until the present time, collected the following plants: and many of the specimens passed through my hands while in the State Botanist's office at Albany, New York. The major part of the species here listed were collected between Orient and Greenport; where much of the region remains in its original state. A few of the best of the specimens are preserved in the New York State Herbarium; some of the poorer ones were saved; "scrappy" ones were consigned to the waste basket; and several fungi were left unnamed or in doubt.

The arrangement of the list accords with Dr. Smith Ely Jelliffe's, The Flora of Long Island, I899. The nomenclature of the Myxomycetes follows Dr. Thomas H. Macbride's, North American Slime-Moulds; and that of the Algae, Dr. Bradley M. Davis's, A Catalogue of the Marine Flora of Woods Hole and Vicinity. The nomenclature of the Fungi in most instances follows P. A. Saccardo's, Sylloge Fungorum: and the authors are under great obligation to Dr. Charles H. Peck, State Botanist of New York, for helping solve many difficult problems among the Higher Basidiomycetes. The nomenclature of the Lichens conforms with a modification of the Nylander system; that of the Hepaticae, follows Dr. A. W. Evans: and that of the Musci, Dr. A. J. Grout's, Mosses with Hand-lens and Microscope. The nomenclature of the ferns and flowering plants follows the last edition of Dr. N. L. Britton and A. Brown's, An Illustrated Flora of the Northern States and Canada.

It has seemed best not to arrange the long list of cryptogams according to families and increase the length of this article: but to list the species under botanical orders alphabetically. The ferns and phanerogams are arranged according to families: but the species are listed in alphabetical order. 


\section{INSECT GALLS*}

Acarus Serotinae Beutm.-Wild Cherry Pouch Gall.

Acraspis erinacei Walsh-Oak Hedgehog Gall.

Amphibolips confluentus Harr.-Oak Apple.

Andricus futilis O. S.-Oak Wart Gall on leaves of Quercus alba.

A. palustris O. S.-Succulent Oak Gall on leaves of Quercus palustris.

A. petiolicola Bass.-Oak Petiole Gall on leaves of Quercus alba and Q. velutina.

A. punctatus Bass.-Oak Knot Gall.

A. seminator Harr.-Oak Seed Gall.

Asteromyia rubra Felt-On leaves of Solidago bicolor.

A ulax tumidus Bass.-Lettuce Tumor Gall.

Biorhiza forticornis Walsh-Oak Fig Gall on twigs of Quercus alba.

Cecidomyia Caryaecola O. S.--Hickory Seed Gall.

C. holotricha O. S.-Hickory Onion Gall.

C. niveipila O. S.-Oak Fold Gall.

C. Nyssaecola Beutm.-On leaves of Nyssa sylvatica.

C. (?) ocellaris Comstock-Maple Spot Gall on leaves of Acer rubrum.

C. sanguinolenta O. S.-Hickory Cone Gall.

C. Serotinae O. S.-Wild Cherry Bud Gall.

Cincticornia pilulae Walsh-Oak Pill Gall on leaves of Quercus velutina.

Cynips Pisum Fitch-Oak Pea Gall.

Cystiphora canadensis Felt-On leaves of Nabalus.

C. viburnifolia Felt-On leaves of Viburnum acerifolium and $V$. dentatum.

Dasyneura Galii Felt-On flower buds of Galium.

D. Salicifolia Felt-On young willow leaves.

Diastrophus Cuscutaeformis O. S.-Blackberry Seed Gall.

D. nebulosus O. S.-Blackberry Knot Gall.

D. niger Bass.-On leaves of Potentilla canadensis.

Dryophanta polita Bass.-Polished Oak Gall.

Erineum fagineum Pers.-Beechleaf Mite Gall.

Eriophyes abnormis Garman-A mite gall on leaves of Tilia americana.

E. Nyssae Trotter-On leaves of Nyssa sylvatica.

E. Querci Garman-On leaves of Quercus velutina.

Gnorimoschema gallaesolidaginis Riley-On stems of Solidago serotina.

Holcaspis globulus Fitch-Oak Bullet Gall.

Hormaphis Hamamelidis Fitch-Witch Hazel Cone Gall.

H. spinosus Shiner-Spiny Witch Hazel Gall.

Hormomyia Crataegifolia Felt-On leaves of Crataegus.

Itonida foliora Russ. \& Hook.-On margin of leaves of Quercus velutina.

Lasioptera farinosa Beutm.-On leaf veins of Rubus.

L. Lycopi Felt-On stems of Lycopus.

L. virginica Felt-On stems of Triadenum virginicum.

L. Vitis O. S.-Grape-vine Tomato Gall.

Nematus pomum Walsh-Willow Apple Gall.

Neolasioptera Erigerontis Felt-On stems of Leptilon canadense.

* The majority of these galls were named by Dr. E. P. Felt, state entomologist of the state of New York. 
$N$. vitinea Felt-On petioles of grape leaves.

Neuroterus batatus Fitch-Oak Potato Gall on twigs of Quercus alba.

$N$. majalis Bass.-On leaves of Quercus alba.

Obolodiplosis Robiniae Hald.-On leaves of Robinia Pseudo-Acacia.

Oligotrophus salicifolius Felt-On leaves of Salix discolor.

Pachypsylla Celtidis-gemma Riley-Hackberry Nodule Gall.

P. Celtidis-mamma Riley-Hackberry Nipple Gall.

Pemphigus Populicaulis Fitch-Poplar Stem Gall.

P. Rhois Fitch-Sumac Tomato Gall on leaves of Rhus glabra.

Phylloxera Caryaecaulis Fitch-Hickory Louse Gall.

$P$. Caryae-globuli Walsh

P. vastatrix Planch.-Grape Phylloxera.

$P$. vitifoliae Fitch

Rhabdophaga Salicifolia Felt-On leaves of Spirea latifolia.

Rhodites bicolor Harr.-Spiny Rose Gall.

R. dichlocerus Harr,-Long Rose Gall.

R. globosus Beutm.-Globular Rose Gall.

R. ignota O. S.-Mealy Rose Gall.

R. verna O. S.-Knotty Rose Gall.

Rhopalomyia anthophila O. S.-On leaves of Solidago.

$R$. capitata Felt-On stems of Solidago.

$R$. lateriflora Felt-On stems of Aster ericoides.

R. racemicola O. S.-On flowers of Solidago rugosa.

Sackenomyia viburnifolia Felt-On leaves of Viburnum dentatum.

Solenozopheria Vaccinii Ashm.-Huckleberry Gall on stems of Gaylussacia baccata. Trypeta polita Lw.-Goldenrod Gall.

\section{THALLOPHYTA}

\section{MYXOTHALLOPHYTA}

\section{MYXOMYCETES}

Arcyria cinerea (Bull.) Pers.-On rotten wood of oak.

A. denudata (L.) Sheldon-On hickory and cherry.

Ceratiomyxa fruticulosa (Muell.) Macbr.-On rotten wood.

Fuligo ovata (Schaeff.) Macbr.-On old oak trunks.

Hemitrichia serpula (Scop.) Rost.-On old hickory wood.

H. vesparium (Batsch) Macbr,-On old hickory wood.

Lycogala epidendrum (Buxb.) Fr.-On pine stumps, hickory and oak.

Plasmodiophora Brassicae Woron.-On roots of Brassica oleracea; determined at

Bureau of Plant Industry, Washington.

Reticularia Lycoperdon Bull.-On Sassafras Sassafras.

Stemonites Smithii Macbr.-On old logs.

Trichia favoginea (Batsch) Pers.-On old wood of Robinia Pseudo-Acacia.

\section{EUTHALLOPHYTA}

Agardhiella tenera (Ag.) J. Ag.

\section{EUPHYCEAE*}

Ahnfeltia plicata (Huds.) Fr.

* The marine algae were mostly determined by Dr. M. A. Howe and are preserved in the Herbarium of the New York Botanical Garden. 
Anabcena torulosa (Carm.) Lag.

Antithamnion Pylaisaei (Mont.) Kjellm.

Ascophyllum nodosum (L.) LeJolis

Callithamnion Baileyi Harv.

C. byssoideum Arn.

Ceramium fastigiatum Harv.

C. rubrum (Huds.) Ag.

C. tenuissimum (Lyngyb.) J. Ag.-Greenport.

Chaetomorpha Linum (Müil.) Kütz.

Champia parvula (Ag.) Harv.

Chlorococcum humicola (Naeg.) Rabenh.-A fresh-water alga on a piece of woor.

Chondria tenuissima (Good. \& Woodw.) Ag.

Chondrus crispus (L.) Stackh.

Chorda filum (L.) Stackh.

Chordaria flagelliformis (Müll.) Ag.

Cladophora arcta (Dillw.) Küitz.

C, expansa (Mert.) Kütz.

C. flexuosa (Griff.) Harv.

C. gracilis (Griff.) Kütz.

Corallina officinalis $\mathrm{L}$.

Cystoclonium purpurascens (Huds.) Kütz.

Dasya pedicellata Ag.

Delesseria sinuosa (Good. \& Woodw.) Lamour.

Dermatolithon pustulatum (Lamour.) Fosl.-On leaves of Zostera.

Desmarestia aculeata (L.) Lamour.

D. viridis (Müll.) Lamour.

Dictyosiphon foeniculaceus (Huds.) Grev.

Ectocarpus confervoides (Roth) LeJolis

E. fasciculatus Harv.

E. siticulosus (Dillw.) Ag.

Enteromorpha compressa (L.) Grev.

E. intestinalis (L.) Grev.

E. $\operatorname{linza}$ (L.) J. Ag.

E. percursa (Ag.) J. Ag.

E. plumosa Kütz.

Epilithon membranaceum (Esp.) Heyd.

Fucus evanescens Ag.

F. spirulis L.

$F$. vesiculosus $\mathrm{L}$.

Gracilaria confervoides (L.) Grev.

Gymnogongrus Torreyi (Ag.) J. Ag.-Dr. Howe says: "probably only a flattened variety of Ahnfeltia plicata (Huds.) Fr."

Halothrix lumbricalis (Kütz.) Reinke-On leaves of Zostera.

Hildebrandia prototypus Nardo

Hypnea musciformis (Wulf.) Lamour.

Isactis glauca Thuret

Laminaria Agardhii Kjellm.

L. digitata (L.) Lamour. 
Leathesia difformis (L.) Aresch.-Probably the young thalli on Zostera.

Lithophyllum pustulatum (Lamour.) Fosl.

Lomentaria uncinata Menegh.

Melobesia Lejolisii Rosan.-On leaves of Zostera.

Mesogloia divaricata (Ag.) Kütz.

Monostroma leptoderma Kjellm.

M. undulatum Farlowii Fosl.

Petalonia fascia (Müll.) Kütz.

Phyllophora membranifolia (Good. \& Woodw.) J. Ag.

Polyides rotundus (Gmel.) Grev.

Polysiphonia elongata (Huds.) Harv.

P. Harveyi Bailey

P. nigrescens (Dillw.) Grev.

$P$. Olneyi Harv.

$P$. urceolata (Lightf.) Grev.

P. urceslata formosa (Suhr) J. Ag.

$P$. variegata (Ag.) Zanard.

Punctaria latifolia Grev.

Pylaiella littoralis (L.) Kjellm.

Rhodermis Georgii (Batt.) Collins-On leaves of Zostera.

Rhodomela subfusca (Woodw.) Ag.-Also the red form, approaching Rhodomela Rochei Harv.

Rhodymenia palmata (L.) Grev.

Sargassum Filipendula (Ag.) J. Ag.

Scytosiphon lomentarius (Lygyb.) J. Ag.

S. lomentarius complanatus Rosev.

Spermothamnium Turneri (Mert.) Aresch.-Attached to the base of Polysiphonia elongata.

Sphacelaria cirrhosa (Roth) Ag.

Spyridia filamentosa (Wulf.) Harv.

Sterrocolax decipiens Schmitz.- "Abundant on Gymnogongrus Torreyi, which fact may be interpreted as strengthening the idea that $G$. Torreyi is only a form of Ahnfeltia plicata."*

Stilophora rhizodes (Ehrh.) J. Ag.

Ulothrix flaccida (Dillw.) Thuret

Ulva Lactuca $\mathrm{L}$.

U. Lactuca latissima (L.) DC.

\section{FUNGI}

SCHIZOMYCETES

Bacillus amylovorus (Burr.) DeToni-On leaves of Pyrus communis.

\section{EUMYCETES}

Phycomycetes

Bremia Laclucae Regel-On Lactuca leaves.

Cystopus candidus (Pers.) Lev.-On Bursa Bursa-pastoris and other mustards.

* Some Midwinter Algae of Long Island Sound, by M. A. Howe in Torreya 14: 97-ror. June, Igr4 (Contr. N. Y. Botanical Garden No. I69). 
Mucor Mucedo L.-On beet stored in a cellar; determined at Cornell by Prof. Fitzpatrick.

Rhizopus nigricans Ehrenb.-On stale bread.

\section{Ascomycetes (excluding Pyrenomycetes)}

Belonidium aurelia (Pers.) DeNot.-On old wood of Robinia Pseudo-Acacia; specimens in N. Y. State Herbarium.

Bulgaria inquinans (Pers.) Fr.

Chlorosplenium aeruginosum (Oeder) DeNot.-On old wood of Acer rubrum.

C. Schweinitzii $\mathrm{Fr}$.

Coccomyces Juniperi Karst.-On bark of Juniperus virginiana; specimens in N. Y. State Herbarium. (Colopoma Juniperinum C. \& P.)

Dasyscypha calcycina (Schum.) Fckl.-On bark of Pinus rigida.

D. virginea (Batsch) Fckl.-On oak.

Discina orbicularis $\mathrm{Pk}$.-On old wood of hickory and cherry.

Durella corrugata (C. \& P.) Sacc.-On oak twigs; specimens in N. Y. State Herbarium.

Exoascus deformans (Berk.) Fckl.-On leaves of Amygdalus persica.

E. varius Atk.-On leaves of Padus virginiana.

Geoglossum velutipes $\mathrm{Pk}$.- On ground in woods.

Geopyxis hesperidea Cke. \& Pk.-On logs; specimens in N. Y. State Herbarium.

Gyromitra esculenta (Pers.) Fr.-Rare in cedar woods at Orient.

Helotium citrinum (Hedw.) Fr.--On old oak and hickory wood.

Helvella macropus (Pers.) Karst.

Humaria granulata (Bull.) Sacc.-On cow dung and old potato stems; specimens in N. Y. State Herbarium.

Lachnea scutellata (L.) Gill.-On old oak wood.

L. setosa (Nees) Gill.-East Marion on rotten stumps.

Lecanidion indigoticum (C. \& P.) Sacc.-Specimens in N. Y. State Herbarium.

Leotia lubrica (Scop.) Pers.

Mollisia melaleuca (Fr.) Sacc.-On old wood of Rohinia Pseudo-Acacia.

Morchella conica Pers.

$M$. deliciosa $\mathrm{Fr}$.

M. esculenta (L.) Pers.-The morels all grow in sandy soil, at Orient.

Phaeangella deformata (Pk.) Sacc. \& D. Sacc.-Growing in lines on bark of Juniperus virginiana; specimens in N. Y. State Herbarium.

Propolis faginea (Schrad.) Karst.--On old wood of willow and hickory; specimens in N. Y. State Herbarium.

Rhytisma acerinum (Pers.) Fr.-Greenport on leaves of Acer rubrum.

R. decolorans Fr.-Greenport on leaves of Xolisma ligustrina and Vaccinium.

$R$. Solidaginis Schw.-On leaves of Solidago.

R. Vitis Schw.-On leaves of grape; determined by Mrs. F. W. Patterson; specimens in N. Y. State Herbarium.

Sarcoscypha occidentalis Schw.

Sclerotinia fructigena (Pers.) Schroet.-On fruit of Amygdalus persica.

S. Libertiana Fckl.-Determined at Bureau of Plant Industry.

Tapesia fusca (Pers.) Fckl.-On oak. 


\section{AsCOMYCETES (PXrenomycetes)}

Botryosphaeria Berengeriana (DeNot.)-On twigs of Toxicodendron radicans.

Calosphaeria Myricae (C. \&. E) E. \& E.-On dead Myrica carolinensis branches; reported in N. X. State Mus. Bull. I67:24. I9I3.

Coronophora angustata Fckl.-On dead Myrica carolinensis trunks; reported in N. Y. State Mus. Bull. I57: 24. I9I2.

Daldinia concentrica (Bolt.) Ces. \& DeNot.-On oak and hickory.

D. vernicosa (Schw.) Ces. \& DeNot.-On old wood of Fagus grandiflora.

Diaporthe parasitica Murrill-On Castanea dentata.

Diatrype albopruinosa (Schw.) Cke.-On Fasus grandiflora.

D. stigma (Hoffm.) Fr.-On old wood of oak.

Diatrypella betulina (Pk.) Sacc.-Greenport.

D. prominens (Howe) E. \& E.-On Plalanus occidentalis.

D. Rhois (Schw.) E. \& E.-On Toxicodendron radicans.

Dothidea Baccharidis Cke.-On dead branches of Baccharis halimifolia; determined by Mrs. F. W. Patterson; reported in N. Y. State Mus. Bull. I67: 26. I9r3.

Eutypella clavulata Cke.-On dead branches of Ailanthus glandulosa.

E. longirostris $\mathrm{Pk}$. - On dead twigs of Robinia Pseudo-Acacia.

E. stellulata (Fr.) Sacc.-On hickory, oak, A melanchier and Robinia Pseudo-Acacia.

Eutypa spinosa (Pers.) Tul.-On old wood of oak.

Gloniopsis australis (Duby) Sacc.-Old wood of oak, Betula populifolia, Sassafras Sassafras and Vitis; specimens in N. Y. State Herbarium.

Glonium parvulum (Ger.) Sacc.-Decorticated wood; reported in N. Y. State Mus. Bull. r50: 45. I9II.

Herpotrichia diffusa (Fckl.) E. \& E.-On decaying wood of Robinia Pseudo-Acacia; reported in N. Y. State Mus. Bull. I50: 45. I9II.

Hypoxylon atropunctatum (Fckl.) Cke.-On old wood of oak.

H. coccineum Bull.-On old wood of oak and Malus Malus.

$H$. fuscopurpureum (Schw.) Berk.-On old wood of hickory.

H. fuscum (Pers.) Fr.--On old wood of Fagus grandiflora.

H. Sassafras (Schw.) Berk.-On old wood of Sassafras Sassafras.

H. serpens (Pers.) Fr.-Greenport on hickory and Quercus alba; specimens in N. Y. State Herbarium.

Hysteriographium Mori (Schw.) Rehm-On old wood of oak, Platanus occidentalis, Malus Malus, Ligustrum vulgare and Baccharis halimifolia.

Hysterium Thujarum Cke. \& Pk.-Determined by Dr. F. J. Seaver.

Hysterium pulicare Pers.--On old wood of Robinia Pseudo-Acacia and Rhus; specimens in N. Y. State Herbarium.

Massaria inquinans (Tode) Fr.

M. vomitoria B. \& C.-On Acer rubrum.

Nectria cinnabarina (Tode) Fr.-Also the conidial form, Tubercularia vulgaris Tode on dead wood of many species of trees and shrubs.

N. episphaeria (Tode) Fr.-Parasitic on Diatrype stigma.

Nummularia clypeus (Schw.) Cke.-On oak stump.

N. discrela (Schw.) Tul.-On old branches of Malus Malus.

Phyllachora graminis (Pers.) Fckl. - On leaves of grasses.

Plowrightia morbosa (Schw.) Sacc.-Branches of Prunus maritima and cultivated auince. 
Rosellinia mutans (C. \& P.) Sacc.-On hickory; specimens in N. Y. State Herbarium.

R. subiculata (Schw.) Sacc.-Greenport on Fraxinus americana.

Sphaerella Fragariae (Tul.) Sacc.-On leaves of Fragaria virginiana.

Ustulina vulgaris Tul.-On oak stumps.

Valsa ambiens (Pers.) Fr.-On dead branches of Fagus grandiflora.

$V$. Linderae Pk.-On dead twigs of Benzoin aestivale; specimens in N. Y. State Herbarium.

V. rhoöphila C. \& E.-On dead branches of Rhus; specimens in N. Y. State Herbarium.

Xylaria polymorpha (Pers.) Grev.-On hickory stump.

\section{HYPOMYCETES}

Alternaria Brassicae (Berk.) Sacc.-On old plants of Ricinus communis and Brassica oleracea; determined at Cornell University.

Cercospora leptosperma Pk.-On leaves of Aralia nudicaulis; determined by Dr. H. D. House.

Illosporium roseum (Schreb.) Mart.-On Physcia stellaris.

Macrosporium commune Rabenh.-On leaves of cucumber and watermelon.

Microstroma Juglandis (Bereng.) Sacc.-On hickory leaves.

Monilia Peckiana S. \& V.-On leaves of Gaylussacia baccata; specimens in N. Y. State Herbarium.

Oospora scabes Thaxt.-Common and very bad on potatoes.

Ovularia obliqua (Cke.) Oud.-On leaves of Rumex.

Pilacre faginea (Fr.) B. \& Br.

Piricularia grisea (Cke.) Sacc.-On leaves of Chaetochloa italica.

Polythrincium Trifolii Kze.-On leaves of Trifolium pratense; determined by Dr. J. C. Arthur.

Ramularia Armoraciae Fckl.-On leaves of Armoracia Armoracia.

R. lineola Pk.-On leaves of Leontodon Taraxacum.

$R$. Plantaginis E. \& M.-The var. nigromaculans Pk., on leaves of Plantago major.

Rhinotrichum ramosissimum B. \& C.-On cherry limbs.

Streptothrix fusca Cda.-On Juniperus virginiana; specimens in N. Y. State Herbarium.

\section{Melanconiales}

Coryneum pulvinatum K. \& S.-On dead branches of Tilia americana.

C. pustulatum Pk.-On dead branches of Castanea sativa; specimens in N. Y. State Herbarium.

Gloeosporium intermedium hrevipes Sacc.-On leaves of the cultivated rubber-plant. (Gloeosporium elasticae Cke. \& Mass.)

Pestalozzia adusta E. \& E.-On leaves of Padus virginiana.

$P$. funerea Desm.-On dead twigs of Juniperus virginiana.

P. longiseta Speg.-On leaves of Rubus and Aronia. The three species of Pestalozzia are reported in N. Y. State Mus. Bull. I57: 30. I9I2.

Steganosporium fenestratum (E. \& E.) Sacc.-On dead branches of Clethra alnifolia; reported in N. Y. State Mus. Bull. 157: 34. I9I2. 


\section{SPHAEROPSIDEAE}

Amerosporium oeconomicum Ell. \& Tracy-On bean leaves; determined by Mrs. F. W. Patterson.

Ascochyta Rhei E. \& E.-On leaves of Rheum rhaponticum; reported in N. Y. State Mus. Bull. 157:22. I9I2.

Camarosporium abnorme (Pk.) Sacc.-On stems of Celastrus scandens.

C. Maclurae Pk.-On dead branches of Toxylon pomiferum; Orient is the type station for this species; reported and described in N. Y. State Mus. Bull. I57: 23. I9I2.

Cytospora chrysosperma (Pers.) Fr.-On dead branches of poplar.

C. Salicis (Cda.) Rabenh.-On dead willow branches; reported in N. Y. State Mus. Bull. I57: 25. I912.

Dendrophoma Tiliae Pk.-On Tilia americana; specimens in N. Y. State Herbarium.

Diplodia ruborum (Schw.) Sacc.-On Rubus.

D. virginiana Cke. \& Rav.-On dead twigs of Juniperus virginiana.

Haplosporella Ribis Sacc.-On dead stems of Grossularia; reported in N. Y. State Mus. Bull. 157: 27. I912.

Hendersonia Grossulariae Oud.-On dead and dying stems of Grossularia reclinala; reported in N. Y. State Mus. Bull. I57: 28. I9I2.

H. pubentis Cke.-On dead stems of Sambucus canadensis.

Macrophoma albifructa (Pk.) Berl. \& Vogl.-On dead maple twigs.

M. Juniperina $\mathrm{Pk}$. - On dead Juniperus virginiane twigs; Orient is the type station for this species; reported and described in N. Y. State Mus. Bull. I67: 28. I9I3.

Phlyctaena septorioides Sacc.-On dead stems of Phytolacca americana.

Phoma ampelinum B. \& C.-On grape leaves.

P. Asclepiadea E. \& E.-On stems of Asclepies syriaca; reported in N. Y. State Mus. Bull. 167: 30. I9I3.

P. Baccharidis Brun.-On dead stems of Baccharis halimifolia.

$P$. corylina (Thuem.) Sacc.-On dead stems of Corylus.

$P$. herbarum West.--On dead stems of Lilium, Rheum rhaponticum and Solidago.

P. moricola Sacc.-On dead twigs of Morus.

$P$. sambucina Sacc.-On dead twigs of Sambucus canadensis.

$P$. semiimmersa Sacc.-On dead branches of Crataegus; reported in N. Y. State Mus. Bull. I67: 30. I9I3.

Phoma Smilacis Boy. \& Jacz.-On dead stems of Smilax rotundifolia; reported in N. Y. State Mus. Bull. I57: 32. I9I2.

Phyllosticta Belae Oud.-On leaves of beet.

P. circumscissa Cke.-On leaves of Amygdalus persica.

P. cruenta (Fr.) Kx.-On leaves of Vagnera racemosa; determined by Mrs. Patterson.

P. Digitalis Bell.-On leaves of Diyitalis.

P. Iridis E. \& M.-On leaves of Iris versicolor; determined by Mrs. Patterson.

$P$. Labruscae Thuem.-On leaves of Parthenocissus tricuspidata.

$P$. phomiformis Sacc.-On leaves of Quercus alba.

$P$. rhoicola E. \& E.-On leaves of Toxicodendron radicans; reported in N. Y. State Mus. Bull. I67: 30. I913.

P. Smilacis E. \& M.-On leaves of Smilax rotundifolia; determined by Mrs. Patterson. 
Septoria Dianthi Desm.-On leaves of Dianthus; reported in N. Y. State Mus. Bull. I57: 33. I912.

S. Kalmiaecola (Schw.) B. \& C.-Leaves of Kalmia latifolia at Greenport and East Marion.

S. Oenotherae West.-On leaves of Oenothera.

S. Polygonina Thuem.-On leaves of Persicaria.

S. Ribis Desm.-On leaves of Ribes vulgare.

S. Rubi West.-On leaves of Rubus.

Sphaeropsis Malorum Pk.-On dead branches of Malus Malus.

S. Peckii Sacc.-On dead twigs of Padus virginiana. (Sphaeropsis anomala Pk.)

S. Persicae E. \& B.-On dead branches of Amygdalus persica; specimens in N. Y. State Herbarium.

S. Tiliacea $\mathrm{Pk}$.-On dead branches of Tilia americana.

\section{BASIDIOMYCETES}

\section{USTILAGINACEAE}

Cintractia Junci (Schw.). Trel--On Juncus tenuis.

Urocystis sorosporioides Koern. - On Thalictrum; determined by Dr. G. P. Clinton.

Tolyposporium bullatum (Schroet.) Schroet.-Fruiting panicles of Echinochloa Crus-galli; determined at Bureau of Plant Industry.

Ustilago anomala Kze.-On Tiniaria Convolvulus.

$U$. Avenae (Pers.) Jens.-On panicles of Avena sativa.

$U$. hypodytes (Schl.) Fr.-Probably on Agropyron; determined by Dr. Clinton, who says, "an uncommon smut for this host."

U. Maydis (DC.) Cda.-On Indian corn:

U. Tritici (Pers.) Jens.-On Triticum.

U. utriculosa (Nees) Tul.-On Polygonum.

\section{Melampsoraceae}

Melampsora arctica Rostr.-On leaves of Salix discolor; determined by Dr. J. C. Arthur,* who says, "the most southern collection known for this species." (Uredo Rosirupiana Arth.)

M. Bigelowii Thuem.-Greenport on willow leaves.

Melampsoridium Betulae (Schum.) Arth.-Determined by Dr. Arthur.

\section{COLEOSPORIACEAE}

Coleosporium Solidaginis (Schw.) Thuem.-On leaves of Aster and Solidago; determined in part by Dr. Arthur and Dr. F. D. Fromme.

\section{Puccintaceae}

Aecidium Majanthae Schum.--Orient on leaves of Vagnera racemosa.

A. Uvulariae Schw.-Orient on leaves of Uvularia sessilifolia.

Gymnoconia interstitialis (Schlect.) Lagh.-Orient on leaves of cultivated and wild Rubi.

Gymnosporangium germinale (Schw.) Kern.-The summer stage, Roestelia auran-

* Specimens determined by Dr. J. C. Arthur and Dr. F. D. Fromme are preserved in the Herbarium of Dr. Arthur at Purdue University, Lafayette, Indiana. The authors are indebted to Dr. Arthur, who has read the manuscript of the Rusts, and who made valuable suggestions. 
tiacả Pk., on leaves of Amelanchier, Crataegus and quince. (Gymnosporangium clavipes C. \& P.)

G. Juniperi-Virginianae Schw.-The summer stage, Roestelia pyrata (Schw.) Thaxt., on Malus Malus leaves; and the winter stage on Juniperus virginiana at Orient; specimens in N. Y. State Herbarium. (Gymnosporangium macropus Link.)

G. nidus-avis Thaxt.-The summer stage on leaves of Amelanchier, determined by Dr. Arthur; and the winter stage on Juniperus virginiana at Orient.

Kuehneola obtusa (Strauss) Arth.-On leaves of Polentilla canadensis; determined by both Dr. Arthur and Dr. Fromme.

Puccinia Asparagi DC.-On Asparagus officinalis.

P. Asteris Duby-On Aster novi-belgii; determined by Dr. Arthur.

$P$. Caricis-A steris Arth.-Determined by Dr. Fromme.

$P$. Caricis-Solidaginis Arth-OOn Carex; determined by Dr. Arthur.

$P$. Cicutae Lasch.-On leaves of Cicuta máculata at Orient and Greenport; determined by Dr. Arthur, who says this species has been previously collected in "three widely separated localities, Florida, Nova Scotia and Nevada.'

$P$. coronata Cda.-On leaves of Rhamnus cathartica and Nothoholcus lanalus; determined by Dr. Arthur; also on leaves of Avena sativa. (Puccinia Rhamni (Pers.) Wettst.)

P. Cyani (Schleich.) Pass.-On Centaurea cyanus; determined by Dr. Arthur.

Puccinia Eleocharidis Arth.-On leaves of Eupatorium; determined by Dr. Arthur.

P. graminis Pers.-On leaves of grasses; the aecia on leaves of Berberis vulgaris.

P. Grossulariae (Pers.) Lagerh.-On Carex virescens; determined by Dr. Arthur.

P. Helianthi Schw.-On leaves of Helianthus.

P. Hieracii (Schum.) Mart.-On leaves of Hievacium Gronovii; determined by Dr. Arthur.

P. Malvacearum Mont.-Orient on leaves of Malva rolundifolia and Althaea rosea.

P. Menthae Pers,-On leaves of Koellia; determined by Dr. Fromme.

P. obscura Schroet.-On Juncoides campestre; determined by Dr. Arthur.

$P$. orbicula Pk. - On leaves of Nabalus trifoliatus; determined by Dr. Arthur.

P. Peckii (DeToni) Kellerman-Orient on leaves of Denothera; determined by Mrs. F. W. Patterson.

P. Pimpinellae (Strauss) Link-Orient on leaves of Washingtonia Claytoni.

P. Pruni-spinosae Pers.-On leaves of Prunus avium and Padus virginiana; determined by Dr. Arthur. (Tranzschelia punclata (Pers.) Arth.)

P. Sambuci (Schw.) Arth.-On leaves of Carex crinita and C. lurida; determined by Dr. Arthur; also the aecia on leaves of Sambucus canadensis at Orient.

P. Seymouriana Arth.-The aecia on leaves of Asclepias pulchra and the telia on Spartina at Orient; determined by Dr. Arthur who says. "in west-central United States the aecia are found also on Apocymum and Cephalanthus."

$P$. Sorghi Schw.-On leaves of sweet corn.

P. Taraxaci Plowr.-On leaves of Leontodon Taraxacum; determined by Dr. Arthur.

P. triticina Erikss.-On various species of Triticum; determined in part by Dr. Arthur. (Puccinia Rubigo-vera (DC.) Wint.)

$P$. uniporula Orton-On Carex virescens; determined by Dr. Fromme.

P. Violae (Schum.) DC.-On leaves of Viola primulifolia at Greenport; determined by Dr. Fromme. 
Uromyces appendiculatus (Pers.) Fr.-On leaves of Strophostyles helvola; determined by Dr. Arthur. (Nigredo appendiculata (Pers.) Arth.)

U. Caladii (Schw.) Farl.-Greenport on leaves of Arisaema triphyllum and Peltandra virginica.

U. Lilii Clinton-On leaves of Lilium; determined by Dr. Arthur. (Nigredo Lilii (Clinton) Arth.)

U. Limonii (DC.) Lev.-On leaves of Limonium carolinianum; determined by Dr. Arthur.

U. Peckianus Farl.-Orient on Distichlis spicata. Doubtless the Aecidium atriplicis Shear, reported in N. Y. State Mus. Bull. I57: 2I. I9I2, on leaves of Atriplex hastata at Orient belongs here. Aecidium atriplicis is a rust which has only been reported on shrubby species of Atriplex in the arid regions of western United States.

Uromyces Silphii (Burrill) Arth.-On Juncus tenuis; determined by Dr. Fromme. (Nigredo Silphii (Burrill) Arth.)

$U$. Spartinae Farl.-Orient on Spartina; determined by Dr. Arthur.

U. Toxicodendri B. \& R.--Orient on leaves of Toxicodendron radicans; determined in part by Dr. Arthur. (Pileolaria Toxicodendri (B. \& R.) Arth.)

\section{TREMELlaCEAE}

Exidia glandulosa (Bull.) Fr.-On old oak and trunks of other trees.

Tremella aurantia Schw.-On bark of Juniperus virginiana.

T. frondosa Fr. - On oak stumps.

Calocera cornea Fr.

\section{DACRYOMYCETACEAE}

Dacryomyces stillatus Nees-On old pine logs.

Guepinia spathularia (Schw.) Fr.-Growing from crevices of an old log.

\section{EXOBASIDIACEAE}

Exobasidium Vaccinii (Fckl.) Woron.-On fruit of Gaylussacia baccata.

\section{THELEPHORACEAE}

Aleurodiscus Oakesii (B. \& C.) Cke.-On the bark of living trunks of Ostrya virginiana.

Corticium effuscatum C. \& E.-On hickory.

C. lacteum Fr.-On cherry and Rhus.

C. roseolum Mass.-On hickory and Vaccinium.

Craterellus cornucopioides (L.) Pers.-Greenport.

Hymenochaete corrugata (Fr.) Lev.-On hickory.

H. Curtisii (Berk.)-On Quercus alba and Padus virginiana. (Stereum Curtisii Berk.)

H. rubiginosa (Schrad.) Lev.-On oak and Castanea sativa logs; specimens in N. Y. State Herbarium.

H. tabacina (Sow.) Lev.-On hickory, Myrica carolinensis and cherry; specimens often resupinate.

Peniophora cinerea (Fr.) Cke.--On dead branches of Betula populifolia, Padus virginiana and maple.

Solenia fasciculata Pers.-On hickory.

S. ochracea Hoffm.-On rotten wood of hickory; specimens in N. Y. State Herbarium. 
Slereum acerinum Pers.-On hickory and maple.

S. acerinum nivosum Rav.-On dead branches of Juniperus virginiana; specimens in N. Y. State Herbarium.

S. complicatum Fr.-On old limbs.

S. frustulosum (Pers.) Fr.-On oak stumps.

S. hirsulum (Willd.) Fr.-On hickory and Nyssa sylvatica.

S. ochraceo-flavum Schw.-On A melanchier.

S. purpureum Pers.-On hickory and Acer rubrum; sometimes resupinate.

S. sericeum Schw.-On oak, Myrica carolinensis and Nyssa sylvatica.

S. spadiceum Fr.-On oak.

S. versiforme B. \& C.-On Hamamelis virginiana.

$S$. versicolor $\mathrm{Fr}$.-On oak.

Thelephora caryophyllea (Schaeff.) Pers.-In cedar woods.

$T$. intybacea Pers.-Greenport in woods.

T. palmata (Scop.) Fr. - The var. americana $\mathrm{Pk}$. in woods.

T. terrestris Ehrh.-In sandy soil.

\section{Clavariaceae}

Clavaria amethystina Bull.-Greenport and Orient.

C. aurea Schaeff.

C. botrytis Pers.-Greenport and Orient.

C. cinerea Bull.

C. corulloides L.-Greenport.

C. cristata Pers.-Greenport.

C. formosa Pers.

C. fusiformis Sow.

C. mucida Pers.-Rotten oak logs on Gardiner's Island.

C. muscoides $\mathrm{L}$.

C. pulchra Pk.

Sparassis crispa (Wulf.) Fr.-Greenport in woods.

\section{HYDNACEAE}

Hydnum aurantiacum (Batsch) A. \& S.

H. chrysocomum Underw.-On oak.

H. farinaceum Pers.-On old hickory wood.

H. ochraceum Pers.-On cherry and Malus Malus; sometimes resupinate.

H. scrobiculatum Fr.-Greenport.

H. spongiosipes $\mathrm{Pk}$.- In cedar woods.

H. subfuscum $\mathrm{Pk}$.

H. velatum B. \& C.-On hickory.

Irpex ambiguus $\mathrm{Pk}$. On pine log.

I. cinnamomeus Fr.-On oak, Fagus grandiflora and Vaccinium.

I. lacteus Fr.-On dead branches of Ilex verticillata.

I. mollis B. \& C.-On cherry.

1. paradoxus (Schrad.) Fr.-On Padus virginiana.

I. sinuosus Fr.-On dead branches of Padus virginiana.

I. Tulipiferae Schw.-On hickory, oak, Malus Malus and Baccharis halimifolia. Phlebia radiata $\mathrm{Fr}$. 


\section{POLYPORACEAE}

Boletus affinis $\mathrm{Pk}$.-Sandy woods.

B. affinis maculosus $\mathrm{Pk}$.

B. auriporus $\mathrm{Pk}$.

B. Ballouii Pk.-Greenport; reported in N. Y. State Mus. Bull. I57: 22. I9I2.

B. castaneus Bull.

B. chrysenteron Fr.

B. felleus Bull.

B. pallidus Frost

B. rubropunctus $\mathrm{Pk}$.

B. scaber Fr.-Greenport.

B. subglabripes Pk.

B. subvelutipes $\mathrm{Pk}$.--Sandy soil.

Daedalea confragosa rubescens (A. \& S.) Pl..-On willow, Betula populifolia, Nyssa sylvatica and Fraxinus americana. (Trametes rubescens (A. \& S.) Fr.)

D. quercina (L.) Pers.-On oak and Padus virginiana.

Daedalea unicolor (Bull.) Fr.-On hickory and oak.

Favolus canadensis Klotsch.- On dead limbs of Fagus grandiflora.

Fomes annosus Fr.-On Juniperus virginiana; specimens in N. Y. State Herbarium.

F. applanatus (Pers.) Fr.-On hickory and cherry.

$F$. carneus Nees-On Juniperus virginiana.

F. rimosus Berk.-On Robinia Pseudo-Acacia; specimens in N. Y. State Herbarium.

$F$. salicinus (Pers.) Fr.-On willow.

Ganoderma pseudoboletus (Jacq.) Murrill-On oak.

Gloeoporus conchoides Mont.-On hickory and oak:

Merulius tremellosus Schrad.-On dead wood of cherry.

M. Ulmi Pk,-On dead Myrica carolinensis; reported in N. Y. State Mus. Bull. I57: 4I. I9I2.

Polyporus betulinus (Bull.) Fr.-On Betula populifolia.

$P$. brumalis (Pers.) Fr.-On oak.

$P$. caesius (Schrad.) Fr.-On Malus Malus.

$P$. chioneus Fr.-On oak and Padus virginiana.

P. cupulaeformis B. \& C.--On Fagus grandiflora.

$P$. epileucus Fr.-On oak.

$\boldsymbol{P}$. fumosus (Pers.) Fr.

$\boldsymbol{P}$. giganteus (Pers.) Fr.-Specimens in N. Y. State Herbarium.

$P$. gilvus Schw.-On Fagus grandiflora, oak, cherry and Xolisma ligustrina (abnormal specimens).

P. hispidus (Bull.) Fr.-On living oak trees.

P. Spraguei B. \& C.-On oak.

$P$. sulphureus (Bull.) Fr.-On stumps.

Polystictus circinatus Fr.-Greenport.

$P$. hirsutus Fr.-On hickory and oak.

$P$. pergamenus Fr.-On oak, cherry and Fagus grandiflora.

$P$. velutinus (Pers.) Fr.-On Xolisma ligustrina.

$P$. versicolor (L.) Fr.-On hickory and oak.

Poria attenuata $\mathrm{Pk}$.-On hickory.

$P$. contigua (Pers.) Fr.-On Robinia Pseudo-Acacia. 
P. ferruginosa (Schrad.) Fr.-On Ilex verticillaia.

P. floccosa Fr.-On oak, Sassafras Sassafras, Malus Malus, Padus virginiana and Acer rubrum.

$\boldsymbol{P}$. inermis E. \& E.-On Ilex verticillata and Toxicodendron radicans; specimens in N. Y. State Herbarium.

$P$. nitida (Pers.) Fr.-On cherry.

$P$. reticulata (Pers.) Fr.-On Juniperus virginiana.

$P$. subacida $\mathrm{Pk}$.

P. subacida vesiculosa (B. \& C.) Pk.-Specimens in N. Y. State Herbarium.

$P$. vulgaris Fr.-On Juniperus virginiana.

Strobilomyces strobilaceus (Scop.) Berk.-Sandy woods.

Trametes cinnabarina (Jacq.) Fr.-On cherry.

T. Pini (Brot.) Fr.-On pine; specimens in N. Y. State Herbarium.

$T$. sepium Berk.-On willow, hickory and cherry.

$T$. suaveolens (L.) Fr.-On willow.

\section{AgARICACEAE}

Agaricus abruptibulbus $\mathrm{Pk}$. - In cedar woods.

A. campester $\mathrm{L}$.

A. micromegethus $\mathrm{Pk}$.- In sandy soil.

A. Rodmani Pk.

Amanita Frostiana Pk.

A. Frostiana pallidipes $\mathrm{Pk}$.

A. glabriceps $\mathrm{Pk}$.

A. muscaria L.-Cedar woods.

A. muscaria formosa (G. \& R.) Fr.

A. phalloides Fr.-A gray variety.

A. verna Fr.

Amanitopsis strangulata (Fr.) Roze

A. vaginata (Bull.) Roze

A. vaginata livida (Pers.) $\mathrm{Pk}$.

Armillaria mellea Vahl

A. nardosmia Ellis-Greenport.

Cantharellus infundibuliformis (Scop.) Fr.

C. minor $\mathrm{Pk}$.

Clitocybe brumalis Fr.- In cedar woods.

C. clavipes (Pers.) Fr.

C. dealbata Sow.

C. odora (Bull.) Fr.

C. pithyophila Fr.-Specimens in N. Y. State Herbarium.

Clitopilus abortivus B. \& C.

C. albogriseus $\mathrm{Pk}$.

C. orcella (Bull.) Fr.

Collybia dryophila (Bull.) Fr.

C. platyphylla Fr.

C. radicata (Relh.) Fr.-A form approaching var. pusilla $\mathrm{Pk}$.

C. tuberosa (Bull.) Fr.-On decaying Lactarius; specimens in N. Y. State Herbarium. 
C. velutipes (Curt.) Fr.

Coprinus micaceus (Bull.) Fr.

C. plicatilis (Curt.) Fr.

Cortinarius heliotropicus $\mathrm{Pk}$,-Greenport.

Entoloma Grayanum Pk.

E. jubatum Fr.

Hygrophorus chlorophanus Fr.

H. laetus (Pers.) Fr.-Specimens in N. X. State Herbarium.

H. miniatus Fr.

H. miniatus subluteus Pk.-Specimens in N. Y. State Herbarium.

H. psittacinus (Schaeff.) Fr.

H. sordidus Pk.-Greenport; reported in N. Y. State Mus. Bull. I57: 28. I9I2. Hypholoma appendiculatum (Bull.) Fr.

H. incertum $\mathrm{Pk}$.

H. perplexum $\mathrm{Pk}$.

H. sublateritium (Schaeff.) Fr.-Growing in clusters about stumps of hickory and oak.

Laccaria amethystina (Bolt.) B. \& Br. (in part)-Oak woods.

L. laccata (Scop.) B. \& Br.

L. trullisata (Ellis) Pk.-In clean sand. (Clitocybe trullisata Ellis.)

Lacturius Peckii Burl.

L. piperatus (Scop.) Fr.

L. serifuus (DC.) Fr.

L. subdulcis (Bull.) Fr.

L. theiogalus (Bull.) Fr.

L. vellereus Fr.

L. volemus Fr.

Lentinus tigrinus (Bull.) Fr.-On dead trees; the diseased form which is known as Lentodium squamulosum Morg.

Lenzites betulina (L.) Fr.-On oak; specimens in N. Y. State Herbarium.

L. sepiaria Fr.-On pine (poroid form), Juniperus virginiana, Picea mariana and cherry; specimens in N. Y. State Herbarium.

L. trabea (Pers.) Fr.-On pine; reported in N. Y. State Mus. Bull. I67: 27. I9г3. Lepiota americana $\mathrm{Pk}$.

L. clypeolaria (Bull.) Fr.

L. naucinoides $\mathrm{Pk}$.-Cultivated fields.

Marasmius androsaceus (L.) Fr.

M. biformis $\mathrm{Pk}$.-Swampy places.

$M$. capillaris Morg.-On grass stems.

$M$. elongatipes $\mathrm{Pk}$.

M. epiphyllus Fr.-On old leaves; reported in N. Y. State Mus. Bull. I57: 28. I9I2.

$M$. insititius Fr.-On leaves and twigs.

M. oreades Fr.

$M$. ramulinus $\mathrm{Pk}$.-On branches in cedar woods.

M. salignus $\mathrm{Pk}$.-On leaves and twigs.

M. salignus major $\mathrm{Pk}$.

M. spongiosus B. \& C.-Specimens in N. Y. State Herbarium. 
M. subnudus (Ellis) Pk.

$M$. velutipes B. \& C.-On leaves.

Mycena atroalboides $\mathrm{Pk}$.

M. corticola (Schum.) Fr.-On bark of Robinia Pseudo-Acacia and grape vines. M. epipiergyria (Scop.) Fr.

M. galericulata (Scop.) Fr.-On logs.

M. sanguinolenta (A. \& S.) Fr.-Specimens in N. Y. State Herbarium.

Omphalia campanella (Batsch) Fr.-On decaying coniferous wood and moss.

Panaeolus papilionaceus Fr.

$P$. retirugis Fr.-Cultivated fields.

P. semiglobalus Murrill-Determined by Dr. W. A. Murrill.

Panus rudis Fr.-On old oak wood.

P. stypticus (Bull.) Fr.-On oak and Amygdalus persica stumps.

P. torulosus Fr.-On Baccharis halimifolia.

Pholiota praecox (Pers.) Fr.

Pleurolus ostreatus (Jacq.) Fr.-On old poplar logs.

$P$. septicus Fr.

Pluteus cervinus (Schaeff.) Fr.

Psathyrella disseminata (Pers.) Fr.

Russula compacta Frost

R. emetica Fr.

$R$. foetens (Pers.) Fr.-Southold.

R. foetentula $\mathrm{Pk}$.-Southold.

R. Mariae Pk.

R. obscura Rom.-Greenport.

$R$. pectinatoides $\mathrm{Pk}$.

R. purpurina Q. \& S.--Orient and Greenport; specimens in N. Y. State Herbarium.

$\boldsymbol{R}$. virescens (Schaeff.) Fr.-Greenport and Orient.

Schizophyllum commune Fr.-On hickory, cherry, Castanea sativa and Baccharis halimifolia.

Siropharia aeruginosa (Curt.) Fr.-On decaying wood.

S. semiglobata (Bastch) Fr.-On horse manure.

Tricholoma alboflavidum $\mathrm{Pk}$.

$T$. personatum Fr.-In oak woods.

T. piperatum Pk.-Reported in N. Y. State Mus. Bull. I67: 32. I913.

T. resplendens Fr.

T. Russula (Schaeff.) Fr.

T. sejunctum Sow.

Trogia crispa (Pers.) Fr.-On oak.

\section{Gasteromycetes}

Anthurus borealis Burt-Orient in corn fields.

Borista pila B. \& C.-In woods and fields.

B. plumbea Pers.

Culvatia craniiformis (Schw.) Morg.-In cedar woods; reported in N. Y. State

Mus. Bull. I50: 24. I9II.

C. cyathiformis (Bosc) Morg.

C. rubroflava (Cragin) Morg.-Sandy soil; reported in N. Y. State Mus. Bull. I67:

24. I9I3. 
Crucibulum vulgare Tul.-On hickory.

Cyathus stercoreus (Schw.) DeToni

C. vernicosus (Bull.) DC.-Sandy soil.

Geaster hygrometricus Pers.

G. minimus Schw.-Cedar woods.

Lycoperdon atropurpureum Vitt.

L. cruciatum Rostk. - In cultivated fields and open woods.

L. echinatum Pers.-Greenport.

L. pusillum Batsch

L. pyriforme Schaeff.-On decayed cherry logs.

L. Wrightii B. \& C.-Greenport.

Phallus impudicus L.

Polysaccum pisocarpium Fr.-Southold and Orient on the ground in woods; reported in N. Y. State Mus. Bull. I57: 32. I9I2.

Scleroderma flavidum E. \& E.-In cedar woods; specimens in N. Y. State Herbarium.

S. Geaster Fr.-In sandy soil; specimens in N. Y. State Herbarium.

S. tenerum B. \& C.-In cedar woods.

S. verrucosum (Bull.) Pers.-Greenport.

S. vulgare Hornem.--In cedar woods; specimens in N. Y. State Herbarium.

\section{LICHENES}

Arthonia lecideella Nyl.-On bark of Tilia americana; determined by Dr. BruceFink.

A. quintaria Ny1.-On Ailanthus glandulosa and Rhus bark; determined by G. K. Merrill; reported in N. Y. State Mus. Bull. I67: 23. I9I3.

A. radiata (Pers.) Th. Fr.-On hickory, Fagus grandiflora, A melanchier, Acer rubrum and Tilia americana bark. (Arthonia astroidea Ach.)

Baeomyces roseus Pers.-East Marion on earth in woods.

Biatora flexuosa Fr.--Dead limbs of Juniperus virginiana; determined by Dr. H. E. Hasse.*

B. varians (Ach.) Tuck.-Determined by Dr. Hasse:

B. vernalis (L.) Fr.-On cedar fence rails; determined by Dr. Hasse.

Biatora (§ Biatorina) cyrtella (Ach., Nyl.) Tuck.-Determined by Dr. Hasse.

Biatora ( $\$$ Bacidia) rubella (Ehrh.) Rabenh.-On bark of Juniperus virginiana and Toxicodendron radicans; determined by Dr. L. W. Riddle.

B. (§Bacidia) Schweinitzii Fr.-On Juniperus virginiana; determined by Dr. Hasse.

Biatora ( $\$$ Biatorella) simplex (Dav.) -On rocks; determined by Dr. Hasse.

Cetraria Islandica (L.) Ach.--Partly determined by Dr. Hasse.

Cladonia alpestris (L.) Rabenh.

C. bacillaris clavata (Ach.) Wainio-On pine bark and Juniperus virginiana stumps.

C. Boryi Tuck., forma reticulata (Russell) Merrill-Specimens in N. Y. State Herbarium.

C. caespilicia (Pers.) Flk.-Sandy soil at East Marion.

C. cariosa cribosa (Wallr.) Wainio-On earth.

C. cristatella Tuck.-On old coniferous logs.

* Specimens of the Lichens named by Dr. Hasse are preserved in the Lichen

Herbarium of The Sullivant Moss Society. 
C. cristalella ochrocarpia Tuck.-Determined by Dr. Hasse.

C. cristalella vestila Tuck.-Greenport; reported in N. Y. State Mus. Bull. I67: 34. I9I3.

C. digitata (Ach.) Schaer.-Light soil in open woods at Orient and East Marion; determined by Dr. Hasse.

C. digitala ceruchoides Wainio-Determined by Dr. Hasse.

Cladonia fimbriata radiata (Schreb.) Fr.-Sandy soil; determined by Dr. Hasse.

C. furcata (Huds.) Schrad.-On earth.

C. furcata pinnata (FIk.) Wainio, f. foliolosa Del.-Determined by Dr. Hasse.

C. furcala racemosa (Hoffm.) Flk.-Determined by Dr. Hasse.

C. gracilis dilatata (Hoffm.) Wainio-Sandy soil; determined by Dr. Riddle; reported in N. Y. State Mus. Bull. I67: 34. I9I3.

C. macilenta (Ehrh.) Hoffm.-A composite species on coniferous wood; partly determined by Dr. Hasse.

C. mitrula Tuck.

C. pyxidata chlorophaea (Spreng.) Flk.-Determined by Dr. Hasse.

C. pyxidata neglecta (Flk.) Mass.-On limbs of Juniperus virginiana and on earth.

C. pyxidata neglecta, m. lophyra Ach.-Determined by Dr. Hasse.

C. rangiferina (L.) Weber

C. squamosa (Scop.) Hoffm.-A variety of this composite species; determined by Dr. Hasse.

C. sylvatica (L.) Rabenh.

C. turgida conspicua (Schaer.) Nyl.-Determined by Dr. Hasse.

C. uncialis (L.) Fr.-A variety of this composite species.

C. verticillata Hoffm.

Collema ( $\$$ Synechoblastus) nigrescens (Huds.) Ach.-On Juniperus virginiana trees; determined by Dr. Hasse.

Graphis dendritica Ach., f. obtusa Leight.-On bark of Acer rubrum; determined by Dr. Hasse.

G. scripta (L.) Ach.-On hickory, oak and Fagus grandiflora bark.

G. scripta recta (Humb.) Koerb.-Determined by Dr. Hasse.

G. scripta serpentina (Ach.) Nyl.--Determined by Dr. Hasse.

Lecanora ( $\$$ Candelaria) laciniosa (Duf.) Nyl.-On Juniperus virginiana and Nyssa sylvatica; also old leather. (Teloschistes concolor (Dicks.) Tuck.)

L. ( $\$$ Candelaria) laciniosa eff use (Tuck.) n. comb.-Reported in N. Y. State Mus. Bull. I50: 49. I9rI.

Lecanora ( $\$$ Callopisma) camptidia (Tuck.) Nyl.-On oak bark at Orient; determined by Dr. Riddle; reported in N. Y. State Mus. Bull. I67: 30. I913. (Placodium camptidium Tuck.)

.L. ( $($ Callopisma) cerina (Ehrh.) Ach.-On Juniperus virginiana; determined by Dr. Fink; specimens in N. Y. State Herbarium.

Lecanora (§Callopisma) ferruginea discolor (Willey) n. comb.-On Juniperus virginiana; determined by Dr. Fink; reported in N. Y. State Mus. Bull. I50: 37. I9I I.

L. ( $\$$ Callopisma) ferruginea nigricans (Tuck.) Nyl.-Determined by Dr. Hasse.

Lecanora (§ Rinodina) ascociscana (Tuck.) Nyl.-Bark of trees; determined by Dr. Hasse.

L. (§ Rinodina) exigua (Ach.) Th. Fr.-Determined by Dr. Hasse. 
L. (§ Rinodina) sophodes (Ach.) Koerb.-On bark of Baccharis halimifolia; determined by Dr. Riddle.

Lecanora Hageni Ach.—Determined by Dr. Hasse.

L. pallida (Schreb.) Tuck.-On bark of Ilex verticillata.

L. pallida cancriformis (Hoffm.) Tuck.-On bark of oak and Amelanchier; determined by both Dr. Fink and Dr. Riddle.

L. subfusca (L., Nyl.) Ach.-On bark of oak, Juniperus virginiana and Robinia Pseudo-Acacia; partly determined by Dr. Fink.

L. subfusca allophana Ach.-On limbs of Juniperus virginiana; determined by Dr. Riddle; specimens in N. Y. State Herbarium.

L. subfusca rugosa (Pers.) Cromb.-Determined by Dr. Hasse.

L. symmictera Nyl.-Determined by Dr. Hasse.

L. varia (Ehrh.) Ach.-On bark of Juniperus virginiana and Pyrus communis.

L. varia saepincola Fr.-On old chestnut fence post; reported in N. Y. State Mus. Bull. I 50: 46. I9II.

Lecanora ( $\$$ Aspicilia) cinerea (L.) Sommf.—On rocks.

Lecanora ( $\$$ Acarospora) cervina squamulosa $\mathrm{Fr}$.-Determined by Dr. Hasse.

L. ( $($ Acarospora) fuscata (Schrad.) Fr.-On rocks; determined by Dr. Hasse.

Lecidea ( $\$$ Buellia) disciformis (Fr.) Nyl.-On oak, Amygdalus persica, Robinia Pseudo-Acacia and Sambucus canadensis bark; Greenport on bark of Fagus grandiflora; partly determined by Dr. Hasse.

L. ( $\S$ Buellia) myriocarpa (DC.) Nyl.-On pine, Juniperus virginiana and Rhus bark; partly determined by Dr. Fink, also Dr. Hasse; specimens in N. Y. State Herbarium.

Lecidea albocaerulescens (Wulf.) Schaer.-Determined by Dr. Hasse.

Leptogium tremelloides (L. f.) S. F. Gray-On Juniperus virginiana.

Myriangium Duriaei (Mont. \& Berk.) Tuck.-On bark of Fraxinus americana at East Marion; determined by Dr. Hasse.

Opegrapha herpetica Ach.-On bark of Tilia americana; determined by Mr. Merrill; reported in N. Y. State Mus. Bull. I67: 29. I9I3.

o. varia Pers.-On bark of hickory, Ailanthus slandulosa and Tilia americana; in part determined by Dr. Riddle.

O. varia pulicaris (Ach.) Nyl.-Determined by Dr. Hasse.

Opegrapha vulgata Ach.-Determined by Dr. Hasse.

Pannaria luridum (Mont.) Nyl.--Rare at Orient on bark of Juniperus virginiana; determined by Dr. Fink; specimens in N. Y. State Herbarium. (Physma luridum (Mont.) Tuck.)

Parmelia caperata (L.) Ach.-On Juniperus virginiana.

$P$. colpodes Ach.-Rare at Orient; specimens in N. Y. State Herbarium.

$P$. conspersa (Ehrh.) Ach.-On rocks.

$P$. crinita pilosella (Hue) Merrill-On Juniperus virginiana.

P. dubia (Wulf.) Schaer.-On trunks of oak. (Parmelia Borreri Tuck.)

$\boldsymbol{P}$. dubia hypomela (Tuck.) n. comb.-On Juniperus virginiana; reported in N. Y. State Mus. Bull. I50: 47. I9II.

P. exasperata (Ach.) DN.-Determined by Dr. Hasse. (Parmelia olivacea aspidota Ach.)

$P$. hyperopta Ach.-Determined by Dr. Hasse.

$P$. olivacea (L.) Ach.-On oak bark. 
$P$. perforata (Jacq.) Ach.-On oak; specimens in N. Y. State Herbarium.

$P$. perforata hypotropa (Nyl.) Tuck.-On Juniperus virginiana; determined by Dr. Fink; reported in N. Y. State Mus. Bull. I50: 47. I9II.

P. rudecta Ach.-On Juniperus virginiana; specimens in N. Y. State Herbarium. (Parmelia Borreri rudecta (Ach.) Tuck.)

$P$. saxatilis (L.) Fr. -On rocks.

P. sulcata Tayl-Dpecimens in N. Y. State Herbarium. (Parmelia saxatilis sulcala Nyl.)

P. tiliacea Ach.-On trunks of Malus Malus.

Pelligera canina (L.) Hoffm.- On earth; determined by Dr. Hasse.

Pertusaria communis Lam. \& DC.-On hickory, oak and Fagus grandiflora; determined by both Dr. Fink and Mr. Merrill.

P. leioplaca (Ach.) Schaer.-On Quercus velutina; determined by Mr. Merrill; specimens in N. Y. State Herbarium.

$P$. multipuncta (Turn.) Nyl.-On hickory; in part determined by Dr. Hasse.

$P$. pustulata (Ach.) Nyl.-Orient and Greenport on Myrica curolinensis; determined by Mr. Merrill.

P. velata (Turn.) Nyl.-Determined by Dr. Fink.

P. Wulfenii Lam. \& DC.-On oak at East Marion; determined by Dr. Hasse.

Physcia ( $\$$ Xanthoria) parielina (L.) DN.-Determined by Dr. Hasse. (Teloschistes parielinus (L.) Norm.).

P. (\$ Xanthoria) parietina aureola (Ach.) Nyl--On Robinia Pseudo-Acacia; determined by Dr. Hasse.

P. ( $($ Xanthoria) polycarpa (Ehrh.) Nyl.-Determined by Dr. Hasse.

Physcia ( $\$$ Pseudophyscia) hypoleuca (Muhl.) Tuck.-Rare at Orient on Juniperus virginiana bark; determined by Miss Mary F. Miller; specimens in N. Y. State Herbarium.

P. ( $\$$ Pseudophyscia) speciosa (Wulf.) Nyl.-Determined by Dr. Hasse.

Physcia hispida (Schreb.) Tuck.-Orient, very rare on a Juniperus virginiana stump; determined by Dr. Fink; reported in N. Y. State Mus. Bull. I50: 36. I9II.

P. obscura (Ehrh.) Ny1.-On bark of Juniperus virginiana; determined by Dr. Hasse.

$P$. obscura endochrysea (Hampe) Nyl.-On Juniperus virginiana.

P. stellaris (L.) Nyl.-On Juniperus virginiana; specimens in N. Y. State Herbarium.

P. iribacia (Ach.) Nyl.-On Juniperus virginiana and Robinia Pseudo-Acacia; also on rocks.

Platysma aurescens (Tuck.) Nyl.—Determined by Dr. Hasse. (Cetraria aurescens Tuck.)

$P$. ciliare (Ach.) Nyl.

P. Fendleri (Tuck.) Nyl.-Determined by Dr. Hasse.

P. glaucum (L.) Nyl.-Determined by Dr. Hasse.

P. lacunosum (Ach.) Nyl.

Pyrenula nitida Ach.-Greenport on trunks of Fagus grandiflora.

Pyxine sorediata (Ach.) Fr.-On oak, Juniperus virginiana and Robinia PseudoA cacia bark.

Ramalina calicaris (L.) Nyl.-On Juniperus virginiana; in part determined by Dr. Hasse. 
R. calicaris subampliata Nyl.-On Juniperus virginiana; reported in N. Y. State Mus. Bull. I 50: 47. I9II, as Ramalina calicaris fraxinea Fr.

R. subfastigiata Nyl.-On oak, Juniperus virginiana and Robinia Pseudo-Acacia; determined by Dr. Hasse.

R. tenuis Tuck., Merrill-On Juniperus virginiana; reported in N. Y. State Mus. Bull. I50: 38. I9I I, as Ramalina rigida (Pers.) Ach.

Sagedia cestrensis Tuck.-Orient on bark of hickory and Tilia americana; determined by Miss Miller; reported in N. Y. State Mus. Bull. 157: 33. I9I2.

Teloschistes chrysopthalmus (L.) Th. Fr.-Determined by Dr. Hasse, who says, "an unusual form on account of almost entire absence of fibrillae."

T. flavicans (Sw.) Norm.-Orient on Juniperus virginiana; determined by Dr. Fink; reported in N. Y. State Mus. Bull. I50: 39. I9II.

Usnea ceratina Ach.-Determined by Dr. Hasse, who says, "the color of the thallus is unusually dark."

U. florida (L.) Hoffm.-On pine and Juniperus virginiana; determined by Dr. Hasse.

$U$. florida rubiginea $\mathrm{Mx}$. - On Juniperus virginiana.

U. hirta (L.) Hoffm.

U. trichodea Ach.-On Juniperus virginiana; reported in N. Y. State Mus. Bull. I50: 40. I9II.

Xylographa parallela (Ach.) Fr.-Determined by Dr. Hasse.

Anthoceros laevis L.

Hepaticae

Calypogeia Sullivanti Aust.-Determined by G. B. Kaiser.

C. Trichomanis (L.) Cda.-Determined by Miss Annie Lorenz.

Cephalozia curvifolia (Dicks.) Dumort.- On an old log in wet woods at Greenport; determined by Dr. G. B. Conklin.

Frullania Asagrayana Mont.-On a rock in swampy woods; determined by both Dr. Conklin and Mr. Kaiser.

F. eboracensis Gottsche-On bark of Juniperus virginiana.

Lophocolea heterophylla (Schrad.) Dumort.-Determined by Dr. Conklin.

Marchantia polymorpha L.-Determined by Dr. Conklin.

Odontoschisma prostratum (Sw.) Trev.-Determined by Miss Lorenz; specimens in N. Y. State Herbarium.

Pellia epiphylla (L.) Cda.-On earth.

Porella pinnata L. - Trunks of bushes in swamps; determined by Mr. Kaiser.

$P$. platyphylla (L.) Lindb.-Trunks of trees.

Ptilidium pulcherrimum (Web.) Hampe

Radula complanata (L.) Dumort.

Ricciella fluitans (L.) A. Br.--On mud in a pond; determined by Mr. Kaiser.

Riccia Sullivantii (Aüst.) Evans.

Ricciocarpus natans (L.) Cda.-Floating in water.

MuscI

Amblystegium riparium B. \& S.--In swamps.

A. riparium longifolium (Schultz) B. \& S.-Determined by G. B. Kaiser.*

A. serpens (L.) B. \& S.

Anomodon attenuatus (Schreb.) Hueb.-About base of trees in woods at Greenport; determined by Mr. Kaiser. 
A. rostratus (Hedw.) Schimp.-Determined by Mr. Kaiser.

Aulacomnium palustre (L.) Schwaegr.-In swamps.

Brachythecium oxycladon (Brid.) J. \& S.-On trunks of trees.

B. rivulare B. \& S.-Wet places.

B. rutabulum (L.) B. \& S.-Sandy soil.

B. velutinum (L.) B. \& S.-On soil at base of trees.

Bryhnia Novae-Angliae (S. \& L.) Grout-Swamps at Greenport.

Bryum caespiticium L.-Greenport; determined by Dr. A. W. Evans.

Campylium hispidulum (Brid.) Mitt.-Determined by Mr. Kaiser.

C. radicale (Bv.) Grout-Determined by Mr. Kaiser.

Catharinea angustata Brid.- - Shaded sandy soil.

C. undulata (L.) W. \& M.

Ceratodon purpureus (L.) Brid.-Sandy soil.

Climacium Kindbergii (R. \& C.) Grout-On roots of trees in wet places; reported in N. Y. State Mus. Bull. I50: 25. I9I.

Dichelyma capillaceum B. \& S.-On Cephalanthus occidentalis in a swamp.

Dicranella heteromalla (L.) Schimp.

Dicranum Drummondii CM.-Wet woods at Greenport; determined by Mr. Kaiser.

D. flagellare Hedw.

D. fulvum Hook.-Determined by Mr. Kaiser.

D. pallidum B. \& S.-Sandy beach in open cedar woods; determined by $\mathbf{M r}$. Kaiser.

D. scoparium (L.) Hedw.

Dilrichum pallidum (Schreb.) Hampe-In cedar woods.

Drepanocladus aduncus gracilescens (Schimp.) - In swamps.

D. fuitans (Dill.) Warnst.-East Marion.

Entodon seductrix (Hedw.) CM.-Wet log in a swamp; determined by Mr. Kaiser Eurhynchium hians (Hedw.) J. \& S.-Sandy soil.

E. serrulatum (Hedw.) Kindb.-Determined by Mr. Kaiser.

E. strigosum (Hoffm.) B. \& S.

Fontinalis Sullivantii Lindb.-Greenport on roots and trunks of bushes in a swamp; determined by Mr. Kaiser.

Funaria flavicans $\mathrm{Mx}$.

$F$. hygrometrica (L.) Sibth.-Greenport; determined by Dr. Evans.

Hedwigia albicans (Web.) Lindb.-Base of oak trees.

Hypnum cupressiforme L.-Determined by Mr. Kaiser.

H. cupressiforme resupinatum (Wils.) Schimp.-Determined by Mr. Kaiser.

H. curvifolium Hedw.

H. Haldanianum Grev.-On old logs and stumps; in part determined by $\mathrm{Mr}$. Kaiser.

H. imponens Hedw.-Moist places.

H. Patientiae Lindb.-Determined by Mr. Kaiser.

H. recurvans (Mx.) Schwaegr.-On moist earth; determined by Mr. Kaiser.

$H$. reptile Mx.-Greenport in moist woods; determined by Mr. Kaiser.

Leptobryum pyriforme (L.) Wils.

* Specimens of the Mosses named by G. B. Kaiser are preserved in the Moss Herbarium of The Sullivant Moss Society. 
Leucobryum glaucum (L.) Schimp.

Leucodon brachypus Brid.-On bark of Juniperus virginiana; determined by $\mathrm{Mr}$. Kaiser.

L. julaceus (Hedw.) Sull.-On Juniperus virginiana bark.

Mniobryum albicans (Wah1.) Limpr.—Determined by Mr. Kaiser.

Mnium affine ciliare (Grev.) CM.-Wet places.

$M$. cuspidatum (L.) Leyss-On rotten logs and moist soil; in part determined by Mr. Kaiser.

M. hornum L.-Edge of a swamp.

M. rostratum Schrad.

Orthotrichum strangulatum Sull.-Greenport on trees; determined by Mr. Kaiser. Physcomitrium turbinatum (Mx.) Brid.-Greenport; determined by Dr. Evans.

Plagiothecium denticulatum (L.) B. \& S.-Wet places at Greenport.

$P$. Ruthei Limpr.-Determined by Mr. Kaiser.

$P$. striatellum (Brid.) Lindb.-Greenport in moist soil; determined by Mr. Kaiser.

P. sylvaticum (Huds.) B. \& S.--Sandy soil; determined by Mr. Kaiser.

$P$. turfaceum Lindb.-On stumps of Juniperus virginiana.

Pleuridium subulatum (L.) Rabenh.-Determined by Mr. Kaiser.

Pogonatum brachyphyllum (Mx.) Bv.-OOrient; determined by G. B. Nichols.

Pohlia nutans (Schreb.) Lindb.-Greenport in light soil in woods; also on sandy beach at Orient; determined by Mr. Kaiser.

Polytrichum commune L.-Dry woods.

$P$. juniperinum Willd.-Determined by G. B. Kaiser.

P. Ohioense B. \& C.-Wet woods at Greenport; determined by Mr. Kaiser.

$P$. piliferum Schreb.

Pylaisia Schimperi R. \& C.

Sphagnum acutifolium Ehrh.-Determined at Bureau of Plant Industry.

S. cuspidatum Ehrh.-Wet open meadow; determined by Mr. Kaiser.

S. cymbifolium Ehrh.-Determined at Bureau of Plant Industry.

S. recurvum Bv.-Determined at Bureau of Plant Industry.

S. Torreyanum Sull.-Boggy woods at Greenport; determined by Mr. Kaiser.

Thelia hirtella (Hedw.) Sull.-Base of oak and other trees.

T. Lescurii Sull.-Sandy soil; determined by Mr. Kaiser.

Thuidium delicatulum (L.) Mitt.

T. paludosum (Sull.) Rau \& Herv.-Wet open soil; determined by Mr. Kaiser.

T. scitum (Bv.) Aust.-Base of oak trees; determined by Mr. Kaiser.

Tortella caespitosa (Schwaegr.) Limpí.-Sandy beach; determined by Mr. Kaiser.

Ulota americana (Bv.) Lindb.-Rocks.

$U$. crispa Brid.-Bark of an old oak; determined by Mr. Kaiser.

Webera sessitis (Schmid.) Lindb.—On rich banks at Greenport.

Weisia viridula (L.) Hedw.-Sandy soil. 
[Reprinted from 'TORREYA, Vol. 14, No. 12, Dec., I9I4.]

\section{PTERIDOPHyTA}

OPHIOGLOSSACEAE

Bolrychium dissechum Spreng.-Rare at Orient and East Marion.

B. neglectum Wood-Rare at East Marion in rich woods.

B. obliquum Muhl,--Rare.

B. virginianum (L.) Sw.

Osmunda cinnamomea L.

OSMUNDACEAE

o. Claytoniana L.

O. regalis $\mathrm{L}$.

\section{Polypodiaceae}

Adiantum pedatum L.- Rare at Orient.

Anchistea virginica (L.) Presl.-Greenport in swamps. (Woodwardia virginica (L.) J. E. Sm.)

Asplenium platyneuron (L.) Oakes-Orient in cedar woods; also the var. serratum (E. S. Miller) BSP.

Athyrium Filix-foemina (L.) Roth-Also the var. latifolium Babingt. (Asplenium Filix-foemina (L.) Bernh.)

Dennstaedia punctilobula (Mx.) Moore.

Dryopteris Clintoniana (D. C. Eaton) Dowell-Rare.

D. cristata (L.) A. Gray-Orient.

D. hexagonoptera $(\mathrm{Mx}$.$) C. Chr.-Rare at Orient; also occurs at Greenport and$ Southold. (Phegopteris hexagonoptera (Mx.) Fee.)

D. intermedia (Muhi.) Gray-Orient.

D. marginalis (L.) A. Gray-Rare at Orient.

D. noveboracensis (L.) A. Gray-Orient.

D. simulata Davenp.-Orient.

D. spinulosa (Muell.) Ktze.-Orient.

D. Thelypleris (L.) A. Gray.

Lorinseria areolata (L.) Presl.-Greenport in swamps. (Woodwardia areolata (L.) Moore.) 
Onoclea sensibilis $\mathrm{L}$.

Polypodium vulgare L.-Mattituck.

Polystichum acrostichoides (Mx.) Schott.-Orient.

Pteridium aquilinum (L.) Kuhn.

Equisetum arvense L.

EQUTSETACEAE

\section{LYCOPODIACEAE}

Lycopodium complanatum L.-Rare at Greenport.

L. inundatum L.-Rare at Southold, the var. Bigelovii Tuck.

L. lucidulum Mx.-Rare at Greenport.

L. obscurum L.--Rare at Greenport, the var. dendrodeum (Mx.) D. C. Eaton.

Selaginellaceae

Selaginella rupestris (L.) Spring.-Rare at Orient in sandy soil, Nov., I9Io and IgII.

\section{SPERMATOPHYTA}

\section{GYMNOSPÉRMAE}

Pinaceae

Juniperus sibirica Burgsd.-Rare at Southold on sandy hills. (Juniperus nana Willd.)

J. virginiana L.-Prostrate forms also occur.

Picea rubens Sargent.-Rare at Orient.

Pinus rigida Mill.

\section{ANGIOSPERMAE}

\section{MONOCOTYLEDONES}

Typha angustifolia $\mathrm{L}$.

TYPHACEAE

T. latifolia L.

\section{SPARGANIACEAE}

Sparganium americanum Nutt.-Rare at Greenport in a stream.

S. androcladon (Engelm.) Morong-Greenport.

S. diversifolium Graeb.-Rare at Southold, 8 Aug., I9Io.

S. eurycarpum Engelm.-Rare on Gardiner's Island.

\section{ZANNICHELLIACEAE}

Potamogeton Oakesianus Robbins-Greenport and Southold in ponds $P$. pusillus L.-Greenport in a shallow pond.

Ruppia maritima L.-Salt-water ponds and creeks.

Zostera marina $\mathrm{L}$

\section{ZOSTERACEAE}

\section{SCHEUCHZERIACEAE}

Triglochin maritima L.-SaIt marshes.

\section{Alismaceae}

Alisma subcordatum Raf.--(Formerly confused with Alisma Plantago-aquatica L.) Sagittaria latifolia Willd.-Rare and variable. 


\section{GRAMINEAE}

Agropyron repens (L.) Bं.--Meadows and cultivated soil. Agrostis alba L.-Also the var. vulgaris (With.) Thurb.

A. canina L.-Orient.

A. hyemalis (Walt.) BSP.-Wooded swamps and brackish meadows; determined in part by Mrs. Agnes Chase.

A. marilima Lam.-Salt marshes; determined in part by Mrs. Chase.

Alopecurus pratensis L.-Rare at Orient in a low field; determined at the New York Botanical Garden.

A mmophila arenaria (L.) Link-Orient.

Andropogon furcatus Muhl.-Orient in shaded places.

A. glomeratus (Walt.) BSP.-Rare at Orient.

Anthoxanthum odoratum L.

Aristida dicholoma $\mathrm{Mx}$.

A. gracilis Ell.-Orient in meadows; specimens in N. Y. State Herbarium.

A. purpurascens Poir-Rare at Gardiner's Island; specimens in N. Y. State Herharium.

Arrhenatherum elatius (L.) Bv.-Determined in part by Mrs. Chase.

Aspris caryophyllea (L.) Nash-Greenport. (Aira caryophyllea L.)

Bromus hordeaceus L. - Orient in sandy soil.

B. purgans L.-Rare at Orient.

$B$. racemosus L.-Rare at Orient.

B. secalinus L.-Cultivated grounds; determined in part by Mrs. Chase.

B. tectorum L.-Rare at Orient in sandy soil.

Calamagrostis canadensis (Mx.) Bv.-Low grounds.

Cenchrus tribuloides L.-Rare at Orient and Gardiner's Island.

Chaetoshloa glauca (L.) Scribn.--Orient in cultivated soil.

C. imberbis (Poir.) Scribn.- Salt marshes.

C. italice (L.) (Ssribn.)-Escaped from cultivation at Orient.

C. viridis (L.) Scribn.

Cinna arundinacea L.-Greenport.

C. latifolia (Trev.) Griseb.-Greenport; specimens in N. Y. State Herbarium.

Cynosorus cristatus L.-Rare at Orient in waste places.

Dactylis glomerata L.-Meadows.

D. anthonic spicata (L.) Bv.-Determined in part by Mrs. Chase.

Deschampsia flexuosa (L.) Trin.

Distichlis spicata (L.) Greene

Echinochloa Crus-galli (L.) Bv.

E. Walteri (Pursh) Nash.

Eleusine indica (L.) Gaertn.

Elymus canadensis L.-Beaches.

E. virginicus L.-Salt marshes.

Eragrostis Eragrostis (L.) Karst.

E. major Host.-Rare at Orient.

E. pectinacea (Mx.) Steud.-Orient on hillsides.

E. pilosa (L.) BV. - Rare at Orient along roads.

Festuca capillata Lam.-Orient in meadows; determined by Mr. P. L. Ricker.

F. elatior L. 
F. nutans Willd.-Orient.

F. octoflora Walt.-Orient in light soil.

$F$. ovina L.-Orient; also the var. pseudovina Hack., on sandy beaches, determined by Mrs. Chase.

Homalocenchrus oryzoides (L.) Poll.-About swamps and ponds.

H. virginicus (Willd.) Britton-Greenport.

Hordeum jubatum L.-Rare at Orient.

Lolium perenne L.-Orient in a meadow.

Muhlenbergia mexicana (L.) Trin.

M. Schreberi Gmeln.-Orient in cedar woods.

M. umbrosa Scribn.-Low woods. (Muhlenbergia sylvatica Torr.)

Nothoholcus lanatus (L.) Nash-(Holcus lanatus L.)

Panicularia acutiflora (Torr.) Ktze.-Orient about ponds.

$P$. grandis (Wats.) Nash-Determined by Mrs. Chase.

$P$. nervata (Willd.) Ktze.-Greenport in wooded swamps; also the var. parviflora (Vasey) in moist woods.

Panicularia pallida (Torr.) Ktze.-Greenport.

P. septentrionalis (Hitchc.) Bicknell-Greenport in wet woods, specimens with very long panicles.

Panicum agrostoides Spreng.-Orient; specimens in N. Y. State Herbarium.

P. amarum Ell.-Southold on sandy beaches; specimens in N. Y. State Herbarium.

$P$. A shei Pearson-Orient; determined by Mrs. Chase.

P. Bicknellii Nash.

P. Boscii Poir.

P. capillare L.-A weed in cultivated soil.

P. cladestinum L.

P. columbianum Scribn.-Determined by Mrs. Chase.

P. Commonsianum Ashe-Determined by Mrs. Chase.

P. depauperatum Muhl.-Sandy soil; determined by Mrs. Chase.

P. dichotomiflorum Mx.-Cultivated soil. (Formerly confused with Panicum proliferum Lam.)

$P$. dichotomum L.-Determined by Mrs. Chase.

$P$. huachucae Ashe-Sandy soil; also the var. silvicola Hitchc. \& Chase, determined by Mrs. Chase.

P. implicatum Scribn.

P. meridionale Ashe-Determined by Mrs. Chase. (Panicum oricola Hitchc. \& Chase.)

P. microcarpon Muhl.-Rare at Greenport, also "a rare form with pubescent spikelets" in cedar woods at Orient; determined by Mrs. Chase. (Panicum barbulatum Nash.)

$P$. polyanthes Schultes-Determined by Mrs. Chase.

P. Scribnerianum Nash-Hilly pastures at East Marion.

P. sphaerocarpon Ell.-Greenport; determined by Mrs. Chase.

P. spretum Schultes-Orient in wet meadows; reported in N. Y. State Mus. Bull. I39: 27. I9I0.

$P$. verrucosum Muhl.-Rare at Orient.

$P$. virgatum L.-Margins of salt marshes; determined in part by Mrs. Chase.

Paspalum circulare Nash-Greenport and East Marion; specimens in N. Y. State Herbarium. 
P. Muhlenbergii Nash.

P. psammophilum Nash.

$P$. setaceum Mx. - Specimens in N. Y. State Herbarium.

Phleum pratense L.

Phragmiles Phragmiles (L.) Karst.-Orient in swamps.

Poa annua L.

$P$. compressa L. - Sandy soil.

$P$. pratensis L.-Meadows.

$P$. triflora Gilib.-Orient about ponds; determined in part by Mrs. Chase. (Formerly confused with Poa flava L.)

Savastana odorata (L.) Scribn.-Wet meadows.

Schizachyrium scoparium (Mx.) Nash-(Andropogon scoparius $\mathrm{Mx}$.)

Sorghastrum nutans (L.) Nash-Orient in moist woods.

Spartina Michauxiana Hitchc.

S. patens (Ait.) Muhl.-Also var. juncea (Mx.) Hitchc.; specimens in N. Y. State Herbarium,

S. stricte (Ait.) Roth-The var. alterniflora (Lois.) A. Gray.

Sphenopolis pallens (Spreng.) Scribn.-Rare at Orient. (Ealonia pennsylvanica (DC.) Gray.)

Sporobolus asper (Mx.) Kunth-Orient.

S. cryplandrus (Torr.) A. Gray-Sandy beaches; reported in N. Y. State Mus. Bull. I50: 49. I9II.

Sporobolus uniflorus Muhl.-Greenport. (Sporobolus serotinus (Torr.) A. Gray.)

S. vaginaeflorus Torr.-Orient.

Stipu avenacea L.-Rare at East Marion and Southold; specimens in N. Y. State Herbarium.

Syntherisma Ischaemum (Schreb.) Nash-(Syntherisma linearis (Krock.) Nash.)

Tridens flava (L.) Hitchc.-Dry soil.

Triplasis purpurea (Walt.) Chapm.-Sandy hills.

Tripsacum dactyloides L.-Upper edge of salt marsh at Orient, I9 July, I909; specimens in N. Y. State Herbarium.

Carex albolutescens Schwein.

Cyperaceae

C. albursina Sheldon-Greenport.

C. blanda Dew.-Orient.

C. canescens L.-Also the var. disjuncta Fernald; specimens of the variety in N. Y. State Herbarium.

C. cephalophora Muhl.-Shaded places.

C. comosa Boott.-Margins of swamps.

C. complanata Torr.-(Carex triceps, var. hirsuta (Willd.) Bailey.)

C. crinita Lam.-Swampy places.

C. digitalis Willd.-Dry open woods.

C. festucacea Schkuhr.-The var. brevior (Dew.) Fernald; determined at Bureau of Plant Industry.

C. Alexuosa Muhl.-Greenport. (Carex tenuis Rudge.)

C. foenea Willd.

C. folliculata L.-Greenport in swampy woods; also a slender form.

C. grisea Wahl.-Determined at Bureau of Plant Industry. 
C. hormathodes Fernald-Wet places.

C. intumescens Rudge-Greenport.

C. lasiocarpa Ehrh.-Greenport. (Carex filiformis L.)

C. Leersii Willd.-Orient in swamps; also Carex stellulata, var. excelsior (Bailey) Fernald.

C. Iupulina Muhl.-Wet woods; also the var. pedunculata Dew.

C. lurida Wahl.-Wet places; also the var. parvula (Paine) Bailey.

C. Muhlenbergii Schkr.-Dry open woods; in part determined at Bureau of Plant Industry.

C. pallescens L.-Orient in moist soil; plants with spikes on longer stalks than usual.

C. pennsylvanica Lam.-Orient and East Marion in dry woods.

C. rosea Schkr.-Hilly woods.

C. rosaeoides E. C. Howe-Orient. (Carex seorsa E. C. Howe.)

C. rostrata Stokes-Wet places. (Carex utriculata Boott.)

C. scoparia Schkr.-Low grounds.

C. silacea Olney-Salt marshes.

C. squarrosa L.-Greenport.

C. stipata Muhl.-Greenport in wet woods.

C. straminea Willd.-Orient.

C. stricta Lam.

C. typhina Mx.-Greenport. (Carex typhinoides Schwein.)

C. varia Muhl.-Greenport.

C. vestita Willd.-Orient in open woods and moist soil.

C. virescens Muhl.-Dry open woods; in part determined at Bureau of Plant Industry.

C. vulpinoidea $\mathrm{Mx}$.-Sandy hillsides and low woods.

C. Willdenowii Schukr.-Determined at Bureau of Plant Industry.

C. dentatus Torr.-Rare at Orient.

C. diandrus Torr.-Wet places and near the beach.

C. esculentus L.-Greenport.

C. $f \operatorname{erax}$ L. C. Richard.--Rare near the beach; specimens in N. Y. State Herbarium.

C. filiculmis Vahl-Sandy hillsides and beaches.

C. filicinus Vahl-Low grounds and beaches; specimens in N. Y. State Herbarium. (Cyperus Nuttallii Eddy.)

C. Grayi Torr.--Southold; specimens in N. Y. State Herbarium.

C. Houghtoni Torr.-Orient.

C. strigosus L.-Low grounds; also the var. capitatus Boeck1.

Dulichium arundinaceum (L.) Britton-Swamps.

Eleocharis acicularis (L.) R. \& S.

E. acuminata (Muhl.) Nees-Greenport.

E. intermedia (Muhl.) Schultes-Greenport.

E. olivacea Torr.-Greenport in a cat-tail swamp; also Orient.

E. obtusa (Willd.) Schultes-In part determined at Bureau of Plant Industry.

E. palustris (L.) R. \& S.-Greenport in wet places.

E. tenuis (Willd.) Schultes-Swampy places.

Eriophorum virginicum L.-Rare at Orient in wet places. 
Fimbristylis autumnalis (L.) R. \& S.-Wet places.

F. castanea (Mx.) Vahl-Rare at Orient on salt marshes; plants with shining(!) scales were collected I4 July I9 3 ; specimens in N. Y. State Herbarium.

Mariscus mariscoides (Muhl.) Ktze.-(Cladium mariscoides (Muhl.) Torr.)

Rynchospora corniculata (Lam.) A. Gray-Rare at Orient, the var. macrostachya (Torr.) Britton.

R. glomerata (L.) Vahl-Low grounds.

Scirpus americanus Pers.--Salt marshes and beaches.

S. alrovirens Muhl.-Orient.

S. cyperinus (L.) Kunth-Wet places in open woods; also Scirpus pedicellatus Fernald.

S. Olneyi A. Gray-Southold; specimens in N. Y. State Herbarium.

S. paludosus A. Nels.-Orient on salt marshes.

S. robustus Pursh.

S. validus Vahl.

Stenophyllus capillaris (L.) Britton-Sandy hillsides.

A corus calamus L.

ARACEAE

Arisaema triphyllum (L.) Torr.

Peltandra virginica (L.) Kunth-Greenport in wet woods.

Spathyema foetida (L.) Raf.-Greenport.

\section{LEMNACEAE}

Lemna minor L.-Greenport, floating on pools.

XYRIDACEAE

Xyris caroliniana Walt.-Southold.

\section{Eriocaulaceae}

Eriocaulon sepiangulare With.-Southold.

\section{Commelinaceae}

Commelina communis L.-Roadsides and waste places.

\section{PONTEDERIACEAE}

Pontederia cordata L.-Greenport and Southold.

Juncoides campestre (L.) Ktze.

JUNCACEAE

Juncus acuminatus $\mathrm{Mx}$.

$J$. articulatus L.-Greenport.

J. balticus Willd.-Brackish meadow at Orient, 30 May, I9Io, and 3 June, I9I4.

$J$. bufonius L.-Margin of salt marshes; specimens in N. Y. State Herbarium.

$J$. canadensis J. Gay.

$J$. dichotomus Ell.-Orient in cultivated field and in swamps, the var. platyphyllus Wieg.; in part determined at Bureau of Plant Industry.

J. effusus L.

J. Gerardi Lois.-Salt marshes; used for hay.

J. Greenei Oakes \& Tuck.-Orient on hills.

$J$. marginatus Rostk.-Rare. 
$J$. pelocarpus E. Meyer-Wet places.

$J$. scirpoides Lam.-Orient.

$J$. secundus Bv.-Orient about wet places.

$J$. tenuis Willd.-Also a few-flowered form; and the var. anthelatus Wieg., determined at Bureau of Plant Industry.

\section{Melanthaceae}

Veratrum viride Ait.-Greenport and Gardiner's Island.

\section{LILIACEAE}

Allium canadense L.-Rare at Orient in dry woods.

A. vineale $\mathrm{L}$.-Orient.

Hemerocallis fulva L.-Orient, escaped along roads.

Lilium canadense $\mathrm{L}$.

L. superbum L.-Orient.

L. tigrinum Andr.-Escaped to roadsides and meadows.

Ornithogalum umbellatum L.-Escaped at Orient.

Asparagus officinalis L.

\section{Convallariaceae}

Polygonatum biflorum (Walt.) Ell.

$P$. commutatum (R. \& S.) Dietr.

Unifolium canadense (Desf.) Greene.

Uvularia perfoliata L.

$U$. sessilifolia $\mathbf{L}$.

Vagnera racemosa (L.) Morong-Some of the plants unusually pubescent (!); specimens in N. Y. State Herbarium.

$V$. stellata (L.) Morong.

Medeola virginiana $\mathrm{L}$.

\section{TRILLIACEAE}

Trillium cernuum L.-Rare at Greenport.

Smilax glauca Walt.

SMtLaceae

$S$. herbacea L.

S. rotundifolia $\mathrm{L}$.

Hypoxis hirsuta (L.) Coville.

\section{Amaryllidaceae}

\section{DIOSCOREACEAE}

Dioscorea villosa L.-Rare on Gardiner's Island and at Southold in moist woods.

\section{IRIDACEAE}

Iris prismatica Pursh-Orient; specimens in N. Y. State Herbarium.

I. versicolor L.

Sisyrinchium angustifolium Mill.

S. atlanticum Bicknell-Orient.

S. graminoides Bicknell-Rare at Greenport in moist woods; determined at Bureau of Plant Industry.

\section{ORCHIDACEAE}

Blephariglottis blephariglottis (Willd.) Rydb.-Orient in bogs; determined at the New York Botanical Garden. 
B. lacera (Mx.) Farwell.

Fissipes acaulis (Ait.) Small-Rare at East Marion and Southold. (Cypripedium acaule Ait.)

Ibidium cernum (L.) House-Orient. (Spiranthes cernua (L.) Richard.)

I. gracile (Bigel.) House.

I. praecox (Walt.) House-Specimens in N. Y. State Herbarium.

Limodorum tuberosum L.-Rare at Orient.

Peramium pubescens (Willd.) MacM.

Perularia flava (L.) Farwell-Rare at Orient; determined at New York Botanical Garden.

Tipularia uniflora (Muhl.) BSP.-Rare at Greenport, 2I Aug. (flowers) and 30 Oct. (fruit), IgIr; "a colony of about 30 plants"; reported in N. Y. State Mus. Bull. I57: 42. I9I2. The I9 July, I9I4, Mr. Latham spent fully two hours hunting for Tipularia before he was successful; so perfectly does the little dark-colored stem blend with the surrounding dead laurel twigs that one almost loses it when they remove the eye from it. The 24 Aug. I9I4, it was decided that a forest fire had destroyed the colony of 30 plants; for the corms were exposed more than one-half out of the ground.

\section{DICOTYLEDONES}

JUGLANDACEAE

Hicoria alba (L.) Britton-Rare; specimens in N. Y. State Herbarium.

H. cordiformis (Wang.) Britton-(Hicoria minima (Marsh.) Britton.)

H. slabra (Mill.) Britton.

H. ovata (Mill.) Britton.

\section{MYricaceae}

Comptonia peregrina (I.) Coult.--East Marion. Myrica carolinensis Mill.

\section{Salicaceae}

Populus alba L.-Roadsides and in woods.

$P$. candicans Ait.-Rarely escaped at Orient.

$P$. deltoides Marsh.-Roadsides and open woods.

$P$. heterophylla L.-Greenport in a swamp.

$P$. grandidentata $\mathrm{Mx}$.-Moist woods.

$P$. italica Moench.- Rarely escaped at Orient.

$P$. tremuloides $\mathrm{Mx}$.

Salis alba L.

S. Bebbiana Sarg.-Wet meadows and swamps.

$S$. cordata Muhl.-Rare at Orient in swamps.

S. discolor Muhl.--Rare at Orient.

S. fragilis L.

S. humilis Marsh.-Dry soil.

S. interior Rowlee-Rare at Orient in wet places. (Salix longifolia Muhl.)

S. lucida Muhl.--Rare at Orient.

S. nigra Marsh.- Rare in low grounds.

S. purpurea L.-Escaped at Orient; specimens in N. Y. State Herbarium.

$S$ sericea Marsh.-East Marion. 


\section{Betulaceae}

Alnus incana (L.) Willd.-Greenport.

A. rugosa (DuRoi) Spreng.-Rare at Orient.

Betula lenta L.-Greenport.

B. populifolia Marsh.

Corylus americana Walt.-Rare at Orient.

C. rostrata Ait.-Orient.

Ostrya virginiana (Mill.) Willd.

Castanea dentata (Marsh.) Borkh.

FAGACEAE

Fagus grandiflora Ehrh.-Greenport, East Marion and Southold; unknown at Orient. (Fagus americana Sweet.)

Quercus alba L:- -More common at Greenport than at Orient.

Q. bicolor Willd.-Greenport.

Q. coccinea Wang.

Q. Muhlenbergii Engelm.-Rare at Greenport.

Q. palustris DuRoi-Rare.

Q. Prinus L.-More common at Greenport than at Oi ient.

Q. rubra L.-Rare.

Q. stellata Wang.-Sandy soil.

Q. velutina Lam.

Celtis occidentalis $\mathbf{L}$.

ULMACEAE

MORACEAE

Toxylon pomiferum Raf.-Escaped from cultivation.

\section{Cannabinaceae}

Humulus Lupulus L.-Rare at Orient in open woods.

\section{URTICACEAE}

Boehmeria cylindrica (L.) Sw.

Pilea pumila (L.) A. Gray.

\section{SANTALACEAE}

Comandra umbellata (L.) Nutt.-Light sandy soil.

\section{ARISTOLOCHIACEAE}

Asarum canadense L.-Determined at New York Botanical Garden.

\section{POLYGONACEAE}

Persicaria Hydropiper (L.) Opiz-Cultivated fields and about yards. (Polygonum Hydropiper L.)

$P$. hydropiperoides (Mx.) Small-About ponds and wet places.

P. lapathifolia (L.) S. F. Gray-Rare at Orient.

$P$. pennsylvanica (L.) Small.

P. Persicaria (L.) Small.

$P$. punctata (Ell.) Small-Fields, waste places and about swamps.

Polygonella articulata (L.) Meisn.--Orient.

Polygonum aviculare L.-Yards and roadsides; sometimes on sandy beaches far from dwellings. 
P. buxiforme Small-Beaches; specimens in N. Y. State Herbarium. (Formerly confused with Polygonum littorale Link.)

$P$. erectum L.-Orient in yards and on beaches.

P. maritimum L. - Beaches on Gardiner's Island; specimens in N. Y. State Herbarium.

P. prolificum (Small) Robins.-Orient; specimens in N. Y. State Herbarium.

$P$. tenue $\mathrm{Mx}$. - Rare at Orient.

Tiniaria Convolvulus (L.) Webb \& Moq.-Plants with the achene more shining than usual (!). (Polygonum Convolvulus L.)

T. dumetorum (L.) Opiz-Rare at Orient.

T. scandens (L.) Small.

Tovara virginiana (L.) Raf.-Gardiner's Island and rare at Greenport. (Poly. gonum virginianum L.)

Tracaulon arifolium (L.) Raf.-Swampy woods. (Polygonum arifolium L.)

T. sagittatum (L.) Small-Gardiner's Island.

Rumex Acetosella L.

R. Brittanica L.-Rare at Greenport in a swamp.

R. crispus L.

R. hastatulus Muhl.

$R$. obtusifolius L.

R. pallidus Bigel.-Rare at Orient; reported in N. Y. State Mus. Bull. I39: 29. I910.

$R$. persicarioides L. - Rare at Orient.

$R$. verticillatus L.-Rare at Greenport.

\section{Amaranthaceae}

Acnida tuberculata Moq.-Rare at Greenport, margin of marshes. Amaranthus blitoides $\mathrm{S}$. Wats.-Rare at Orient in waste places.

A. graecizans $\mathrm{L}$.

A. hybridus $\mathrm{L}$.

A. retroflexus $\mathrm{L}$.

\section{Chenopodiaceae}

Atriplex arenaria Nutt.-Orient on beaches and salt marshes.

A. hastata L.-Beaches and salt marshes; also Atriplex littoralis L., specimens in N. Y. State Herbarium.

Chenopodium album $\mathrm{L}$.

C. ambrosioides $\mathrm{L}$.

C. glaucum L.-Orient, Io Oct. I9I0, a recent introduction from New York City in stable manure.

C. hybridum L.-Orient on sandy beaches and salt marshes.

C. leptophyllum (Moq.) Nutt.-Orient on sandy beaches.

C. urbicum L.-Orient.

Dondia maritima (L.) Druce.

Salicornia ambigua Mx.

S. Bigelovii Torr.

S. europaea L.-The Salicornias determined by Dr. C. H. Peck.

Salsola Kali L.-Specimens in N. Y. State Herbarium. 
Phytolaccaceae

Phytolacca americana L.-(Phytolacca decandra L.)

Mollugo verticillata $\mathbf{L}$.

AIZOACEAE

Sesuvium maritimum (Walt.) BSP.-Gardinet's Island; specimens in N. Y. State Herbarium.

Portulacaceae

Claytonia virginica L.--Rare at East Marion.

Portulaca oleracea L.

Alsinaceae

Alsine graminea (L.) Britton-Rare at Orient.

A. media L.

Arenaria serpyllifolia L.-Orient.

Cerastium viscosum L.-Reported in N. Y. State Mus. Bull. I39: 33. I9Io.

C. vulgatum L.

Honkenya peploides (L.) Ehrh.-Orient on beaches; specimens in N. Y. State

Herbarium. (Arenaria peploides L.)

Moehringia lateriflora (L.) Fenzl.

Sagina decumbens (Ell.) T. \& G.-Rare at East Marion; reported in N. Y. State

Mus. Bull. I50: 48. I9II.

S. procumbens L.

Spergula arvensis L.

Tissa marina (L.) Britton-Specimens in N. Y. State Herbarium.

T. rubra (L.) Britton-Rare at Orient.

Agrostemana Githago L.

\section{Caryophyllaceae}

Dianthus Armeria L.

Lychnis alba Mill.-Roadsides.

L. Coronaria (L.) Desr.-Rare at Orient.

$L$. dioica L. - Rare at Orient.

Saponaria officinalis L.

Silene antrirhina $\mathrm{L}$.

S. caroliniana Walt.-Orient and Southold in sandy soil.

S. latifolia (Mill.) Bitten \& Rendle-Rare.

S. noctiflora L.-Rare at Orient.

S. stellata (L.) Ait.-Rare at Orient.

Vaccaria Vaccaria (L.) Britton-Rare at Orient.

\section{NyMphaEACEAE}

Castalia odorata (Dryand.) Woodv. \& Wood.

\section{Magnoliaceae}

Liriodendron Tulipifera L.-Rare at Greenport.

Anemone quinquefolia L.

Ranunculaceae

$A$. virgiriana $\mathrm{L}$.

Aquilegia canadensis L.-Rare at Orient. 
Caltha palustris L.-Rare at Southold in wet places.

Clematis virginiana $\mathrm{L}$.

Halerpestes Cymbalaria (Pursh) Greene-Waste places at Orient; determined at

Bureau of Plant Industry. (Ranunculus Cymbalaria Pursh.)

Ranunculus abortivus L.-Rare at Orient.

R. acris L.-Rare at Greenport and Southold.

$R$. bulbosus $\mathrm{L}$.

$\boldsymbol{R}$. delphinifolius Torr.-Rare at Orient.

$R$. recurvatus Poir.-Greenport; plants with a bulbous base (!).

$\boldsymbol{R}$. sceleratus L.-Rare at Greenport and Southold in muddy places.

Thalictrum polygamum Muhl.

T. revolutum DC.-Orient. (Thalicirum purpurascens Am. auth.)

Berberis vulgaris L.

BERBERIDACEAE

LAURACEAE

Benzoin aestivale (L.) Nees-Greenport and Gardiner's Island in wet woods.

Sassafras Sassafras (L.) Karst.

\section{PAPAVERACEAE}

Glaucium Glaucium (L.) Karst.-Rare at Orient; but frequent on Gardiner's Island. Papaver Rhoeas L.-Rare at Orient.

$P$. somniferum L.-Rare at Orient.

\section{Cruciferae}

Alyssum alyssoides L.- Rare at Orient; determined at Bureau of Plant Industry. Arabis glabra (L.) Bernh.-Sandy soil (purplish plants).

Armoracia Armoracia (L.) Britton-Rare at Orient. (Roripa Armoriacia (L.) A. S. Hitchc.)

Barbarea Barbarea (L.) MacM.

B. verna (Mill.) Aschers-Rare at Orient; determined at Bureau of Plant Industry. Berteroa incana (L.) DC.-Rare at Orient in meadows.

Brassica campestris L.-Cultivated fields.

B. juncea (L.) Cosson-Rare at Orient.

B. Napus L.

B. nigra (L.) Koch-Rare in waste ground.

Bursa Bursa-pastoris (L.) Britton.

Cakile edentula (Bigel.) Hook.-Upper edge of salt marshes.

Camelina sativa (L.) Crantz-Orient in grain fields; reported in N. Y. State Mus. Bull. I50: 24. I9II.

Cardamine arenicola Britton-Greenport; specimens in N. Y. State Herbarium.

C. pennsylvanica Muhl.-Orient in muddy places.

Draba verna L.-Dry hilly shaded places.

Erysimum officinale L.-(Sisymbrium officinale (L.) Scop.)

Koniga maritima (L.) R. Br.-Rare at Orient.

Lepidium campestre (L.) R. Br.-Meadows.

L. densiflorum Schrad.-Rare at Orient. (Formerly confused with Lepidium apetalum Willd.)

L. sutivum L.-Meadows. 
L. virginicum $\mathrm{L}$.

Norta altissima (L.) Britton-Rare at Orient in meadows. (Sisymbrium altissimum L.)

Radicula palustris (L.) Moench-(Roripa palustris (L.) Bess.)

Raphanus Raphanistrum L.

$R$. sativus L.-Grain fields.

Sinapis arvensis L.-(Brassica arvensis (L.) BSP.)

Sisymbrium Nasturtium-aquaticum L.-Rare at Orient. (Roripa Nasturtium (L.) Rusby.)

Sophia Sophia (L.) Britton-Rare at Orient.

Drosera intermedia Hayne

DROSERACEAE

D. rotundifolia L.-Rare at Southold.

\section{Crassulaceae}

Sedum triphyllum (Haw.) S. F. Gray-Rare. (Sedum telephium Am. auth.)

\section{SAXIFRAGACEAE}

Micranthes virginiensis (Mx.) Small-Rare at Orient. (Saxifraga virginiensis $\mathrm{Mx}$.)

\section{HAMAMELIDACEAE}

Hamamelis nivirgiana L.-Greenport, Southold and Gardiner's Island in moist woods; no Orient records.

\section{Grossulariaceae}

Grossularia Cynosbati (L.) Mill.-O-Orient in woods. (Ribes Cynosbati L.) G. oxyacanthoides (L.) Mill.

Ribes vulgare Lam.-Orient. (Formerly confused with Ribes rubrum L.)

\section{Platanus occidentalis L.}

\section{Platanaceae}

Agrimonia gryposepala Wallr.

\section{ROSACEAE}

Argentina Anserina (L.) Rydb.-Orient and Gardiner's Island on beaches and salt marshes.

Fragaria vesca L.-Woods.

F. virginiana Duchesne-Hills and swamps (variable).

Geum canadense Jacq.

Geum flavum (Porter) Bicknell-Greenport.

G. virginianum $\mathrm{L}$.

Potentilla argentea L.-Orient.

$P$. canadensis $\mathrm{L}$.

P. monspeliensis L.

P. pumila Poir.-Orient and East Marion.

P. simplex $\mathrm{Mx}$.-Orient.

Rosa carolina L.-Sometimes the bushes are almost wholly unarmed.

$R$. cinnamomea $\mathrm{L}$.-Rarely escaped at Orient.

$R$. rubiginosa L.-Sandy soil and shaded places.

R. rugosa Thunb.-Rarely escaped at Orient on sandy beaches; determined at Bureau of Plant Industry. 
R. virginiana Mill.-Both forms occur, Rosa humilis Marsh. and Rosa lucida Ehrh. Rubus alleghaniensis Porter.

$\boldsymbol{R}$. hispidus L.-Sandy bogs.

$R$. occidentalis $\mathrm{L}$.

$\boldsymbol{R}$. phoenicolasius Maxim.-Orient, established in woods.

R. procumbens Muhl.

$R$. strigosus $\mathrm{Mx}$.

Sanguisorba canadensis L.-Greenport in wet woods.

Spirea lalifolia (Ait.) Borkh.-Wet places.

S. tomentosa L.-Orient.

\section{Malaceae}

Amelanchier canadensis (L.) Medic.

Aronia arbutifolia (L.) Ell.-Orient.

A. atropurpurea Britton.

A. melanocarpa (Mx.) Britton.-Orient.

Crataegus chrysocarpa Ashe-Rare at Orient. (Crataegus rotundifolia (Ehrh.) Borkh.)

C. Crus-Galli L.

C. monogyna Jacq.-Rare at Greenport. (Formerly confused with Crataegus Oxyacantha L.)

Malus Malus (L.) Britton.

\section{AMYGDALACEAE}

Padus virginiana (L.) Mill.-(Prunus serotina Ehrh.)

Prunus americana Marsh.-Rare at Orient.

P. Avium L.-Roadsides.

$P$. Cerasus L.-Wood margins.

$P$. domestica L.-Rare, a degenerate form.

$\boldsymbol{P}$. maritima Wang.-Rare at Greenport; but more abundant at Orient, Southold and Gardiner's Island.

\section{Caesalpiniaceae}

Chamaecrista fasciculata (Mx.) Greene.

Gleditschia triacanthos L.-Rare at Orient.

Baptisia tinctoria (L.) R. Br.

\section{FABACEAE}

Coronilla varia L.-Rare at Greenport; specimens in N. Y. State Herbarium.

Craccä virginiana L.-Mattituck.

Falcata comosa (L.) Ktze.-Rare at Orient in rich woods.

Glycine Apios L.-Swamps; plants sometimes with 3 leaflets. (Apios Apios (L.) MacM.)

Lathyrus maritimus (L.) Bigel.

L:spedeza capitata $\mathrm{Mx}$.

L. frutescens (L.) Britton-Southold.

L. hirla (L.) Hornem.

L. procumbens Mx.-East Marion.

L. Stuvei Nutt.-Southold.

L. violacea (L.) Pers.--Greenport and Southold.

L. virginica (L.) Britton-Southold.

Lupinus perennis L.--Southold in sandy soil. 
Medicago hispida Gaertn.-Rare at Greenport.

M. lupulina L.-Greenport.

$M$. sativa $L$.- Rare in meadows.

Meibomia canadensis (L.) Ktze.-Rich woods.

M. Dillenii (Darl.) Ktze-Greenport and Southold in rich woods.

M. grandiflora (Walt.) Ktze.

M. nudiflora (L.) Ktze.-Southold.

$M$. viridiflora (L.) Ktze.-Rare at Greenport; determined at Bureau of Plant Industry.

Melilotus alba Desv.-Greenport.

Phaseolus polystachyus (L.) BSP.-Orient in woods.

Robinia Pseudo-Acacia L.

R. viscosa Vent.

Strophostyles helvola (L.) Britton-Near beaches; specimens in N. Y. State Herbarium.

Trifolium agrarium $\mathrm{L}$.

T. arvense $\mathrm{L}$.

T. hybridum L.-Meadows.

$T$. incarnatum L.-Rare.

T. pratense L.

T. procumbens L.-Rare.

$T$. repens L.

Vicia angustifolia L.-Orient; also the var. segetalis (Thuill.) Koch; reported in , N. Y. State Mus. Bull. I50: 49. I9II.

V. hirsuta (L.) Koch-Rare at Orient; determined at Bureau of Plant Industry.

\section{Gerantaceae}

Erodium cicutarium (L.) L'Her.-Mattituck.

Geranium maculatum L.

Robertiella Robertiana (L.) Hanks-(Geranium Robertianum L.)

Xanthoxalis Brittoniae Small

\section{OXáidDaceaE}

$X$. stricta (L.) Small.

\section{LINACEAE}

Cathartolinum striatum (Walt.) Small-Brackish meadows and wet woods. (Linum striatum Walt.)

C. virginianum (L.) Reichenb.

Linum usitatissimum L.-Rare in grain fields.

\section{BALSAMINACEAE}

Impatiens biflora Walt.-Greenport, Southold and Gardiner's Island in wet places.

\section{Simaroubaceat}

Ailanthus glandulosa Desf.-Rare at Greenport.

Polygala cruciata L.-Orient.

Potygalaceae

$P$. lutea L.-Rare at Greenport. 
$P$. paucifolia Willd.-Orient, the station destroyed by cultivation; determined at New York Botanical Garden.

P. polygama Walt.-Dry open woods.

$P$. verticillata L.-Dry hilly soil.

$P$. viridescens L.-Moist woods.

Acalypha gracilens A. Gray.

\section{EUPHORBIACEAE}

A. virginica L.

Chamaesyce maculata (L.) Small-Cultivated fields. (Euphorbia maculata L.)

C. polygonifolia (L.) Small.

Tithymalus Cyparissias (L.) Hill-Roadsides. (Euphorbia Cyparissias L.)

$T$. Lathyrus (L.) Hill-Rare at Orient in waste places; determined at Bureau of Plant Industry.

\section{CALlitrichaceAE}

Callitriche heterophylla Pursh-Edge of a pond in woods at Greenport; determined at Bureau of Plant Industry.

C. palustris L.- - Rare at Orient in a pond, I908; determined at New York Botanical Garden. Not found since and perhaps the many years of drought have killed the roots.

Rhus copallina L.

\section{Anacardiaceae}

R. glabra L.

Toxicodendron radicans (L.) Ktze.-Plants may be either low or high climbing; the leaves sometimes toothed. (Rhus radicans L.)

T. vernix (L.) Ktze.-Rare.

\section{ILICACEAE}

Ilex bronxensis Britton-Rare at Orient; specimens in N. Y. State Herbarium.

I. glabra (L.) A. Gray-Rare at East Marion.

I. verticillata (L.) A. Gray.

Celastrus scandens L.

\section{Celastraceae}

ACERACEAE

Acer carolinionum Walt.-Rare on Gardiner's Island. (Acer rubrum tridens Wood.)

A. Negundo L.-Escaped at Orient.

A. rubrum L.

A. saccharinum $\mathrm{L}$.

Ceanothus americanus L.--Rare at Southold.

Rhamnus cathartica L.-Orient.

VITACEAE

Parthenocissus quinquefolia (L.) Planch.

Vitis aestivalis $\mathbf{M x}$.

$V$. bicolor LeConte.

V. Labrusca L.-Moist thickets.

Tilia americana L.

\section{TIIIACEAE}

\section{MaLvaCEAE}

Abutilon Abutilon (L.) Rusby-Orient in cultivated fields. Hibiscus Moscheutos L. 
H. Trionum L.-Rare at Greenport.

Malva rotundifolia $\mathrm{L}$.

\section{HYPERICACEAE}

Hypericum canadense L.-Wet meadows; specimens in N. Y. State Herbarium,

H. majus (A. Gray) Britton-Moist places.

H. mutilum L.-Moist places.

H. perforatum $\mathrm{L}$.

H. punctatum Lam.-(Hypericum maculatum Walt.)

Sarothra gentianoides L.

Triadenum virginicum (L.) Raf.

\section{Cistaceae}

Crocanthemum canadense (L.) Britton-East Marion. (Helianthemum canadense (L.) Mx.)

C. majus (L.) Britton-East Marion.

Hudsonia tomentosa Nutt.-Orient, Southold and Gardiner's Island on sandy beaches.

Lechea intermedia Leggett-Orient.

L. Leggettii Britt. \& Holl.-Orient.

L. maritima Leggett.

L. racemulosa Lam.-Orient and Greenport; specimens in N. Y. State Herbarium. L. villosa Ell.-Dry woods.

Viola cucullata Ait.-Swamps.

\section{VIOLACEAE}

V. fimbriatula J. E. Sm.

$V$. lanceolata $\mathrm{L}$,

$V$. pallens (Banks) Brainerd.

$V$. papilionacea Pursh-The var. domestica Bicknt in old lawns.

V. pedata L.-East Marion and Greenport.

V. primulifolia L.-Greenport.

\section{CActaceae}

Opuntia Opuntia (L.) Coult.-Rare at Orient in sandy soil.

Decodon verticillatus (L.) Ell.

\section{LYTHRACEAE}

Rhexia virginica L."-Rare at Orient.

\section{ONAGRACEAE}

Chamaenerion angustifolium (L.) Scop.-Rare at Orient.

Circaea lutetiana $\mathrm{L}$.

Epilobium adenocaulon Haussk.

E. coloratum Muhl.-Orient.

E. lineare Muhl.-Rare at Orient.

E. palustre L.-Rare at Orient.

E. strictum Muhl.-Rare at Orient.

Isnardia palustris L.-Wet places.

Kneiffia Alleni (Britton) Small-Orient.

$K$. fruticosa (L.) Raimann-Also the var. pilosella Britton.

$K$. linearis (Mx.) Spach-Orient.

$K$. longipedicellata Small-Orient. 
K. pumila (L.) Spach.

Ludwigia alternifolia L.-Swampy places.

Oenothera biennis L.

o. Oakesiana Robbins-Orient.

\section{HALORAGIDACEAE}

Myriophyllum humile (Raf.) Morong-Rare at East Marion.

M. pinnatum (Walt.) BSP.-Greenport in a pond; determined by P. L. Ricker.

Proserpinaca palustris L.-Orient.

Aralia nudicaulis L.

Araliaceae

A. racemosa L.-Rare at Greenport.

\section{Ammiaceae}

Angelica alropurpurea L.-Rare at East Marion; specimens in N. Y. State Herbarium.

Cicuta maculata L.

Daucus Carota L.

Foeniculum Foeniculum (L.) Karst.-Escaped at Orient.

Heracleum lanatum $\mathrm{Mx}$.

Ligusticum scoitcum L.-Orient, edge of woods bordering salt marshes; reported in N. Y. State Mus. Bull. ז39:25. I9I0.

Pastinaca sativa L.-Rare.

Plilimnium capillaceum (Mx.) Raf.

Sanicula canadensis $\mathrm{L}$.

S. marylandica L.

Sium cicutaefolium Schrank-Swampy woods.

Washingtonia Claytoni (Mx.) Britton-Orient; specimens in N. Y. State Herbarium.

W. longistylis (Torr.) Britton-Rare.

\section{CORNACEAE}

Cornus femina Mill.-Rare at Orient but more abundant at East Marion and Southold. (Cornus candidissima Marsh.)

C. stolonifera Mx.--Rare at Orient, edge of a swamp.

Cynoxylon floridum (L.) Raf.-(Cornus florida L.)

Nyssa sylvatica Marsh.

Clethra alnifolia L.

Chimaphila maculata (L.) Pursh.

Clethraceae

PYROLACEAE

C. umbellata (L.) Nutt.-Rare at Orient but more common at East Marion. Pyrola americana Sweet.

P. elliptica Nutt.

\section{MONOTROPACEAE}

Hypopitys lanuginosa (Mx.) Nutt.-In oak woods; the plants crimson. Monotropa unifora L.

\section{ERICACEAE}

Azalea nudifiora L.-Rare at Greenport in swamps.

A. viscosa L.-Greenport and Orient in sandy swamps. 
Epigaea repens L.-East Marion.

Eubotrys racemosa (L.) Nutt.-Rare at Greenport in swamps. (Leucothoë racemosa (L.) A. Gray.)

Gaulthera procumbens L.-Rare at Southold.

Kalmia angustifolia L.-Mattituck.

K. latifolia L.-East Marion and Southold.

Uva-Ursi Uva-Ursi (L.) Britton-Orient. (Arctostaphylos Uva-Ursi (L.) Spreng.) Xolisma ligustrina (L.) Britton.

\section{VACCINIACEAE}

Gaylussacia baccata (Wang.) K. Koch-In woods.

Oxycoccus macrocarpus (Ait.) Pursh-Plants bearing two forms of fruit occur; taller plants bear oblong fruit and are rarer.

Vaccinium angustifolium Ait.-(Vaccinium pennsylvanicum Lam.)

$V$. atrococcum (A. Gray) Heller-In swamps.

V. corymbosum L.

V. vacillans Kalm-In woods.

Anagallis arvensis $\mathrm{L}$.

Primulaceae

Lysimachia Nummularia L.-Rare at Orient.

L. quadrifolia L.

L. terrestris (L.) BSP.

Samolus floribundus HBK.-Orient.

Steironema ciliatum (L.) Raf.-Gardiner's Island in low woods; determined by Norman Taylor.

Trientalis americana Pursh.

\section{Plumbaginaceae}

Limonium carolinianum (Walt.) Britton.

\section{OLEACEAE}

Fraxinus americana L.-Greenport.

Ligustrum vulgare $\mathrm{L}$.

Syringa vulgaris L.

\section{Gentianaceae}

Bartonia virginica (L.) BSP.-Rare.

Sabbatia stellaris Pursh-Orient on salt marshes; specimens in N. Y. State Herbarium.

Apocynum androsaemifolium $\mathbf{L}$.

\section{Apocynaceae}

A. cannabinum L.-Rare at Orient; plants with the leaves lightly pubescent beneath.

A. medium Greene-Rare at Orient.

A. pubescens R. Br.-Rare at Orient; specimens in N. Y. State Herbarium.

A. sibiricum Jacq.-(A pocynum album Greene.)

Vinca minor L.-Roadsides at Greenport.

\section{Asclepiadaceae}

Asclepias amplexicaulis J. E. Smith-Rare at Orient.

A. purpurascens L.-Orient. 
A. pulchra Ehrh.-Orient in low ground.

A. syriaca L.-Specimens in N. Y. State Herbarium.

A. tuberosa L.-Rare at East Marion, Greenport and Southold.

A. verticillata L.-Rare at Orient.

Coniolvulus repens L.-Orient.

Convolvulaceae

C. sepium L.-Orient; the flowers white or pinkish.

I pomoea purpurea (L.) Lam.-Escaped.

\section{Cuscutaceae}

Cuscuta arvensis Beyrich-Orient and Southold.

C. compacla Juss.-Greenport on Clethra and Cephalanthus.

C. Gronovii Willd.

\section{Polemoniaceae}

Phlox paniculata L.-Rarely escaped at Orient.

P. subulata L.-The two stations at Orient have recently been destroyed by cultivation.

\section{BORAGINACEAE}

Cynoglossum officinale L.-Rare at Orient.

$M$ yosotis arvensis (L.) Hill-Rare at Orient in cultivated grounds; determined at Bureau of Plant Industry.

$M$. virginica (L.) BSP.-Rare at Orient; specimens in N. Y. State Herbarium.

Onosmodium virginianum (L.) DC.-Rare at Orient on sandy beaches.

Verbena hastata L.

VERBENACEAE

V. urticifolia L.-Shaded places.

\section{LABIATAE}

Agastache nepetoides (L.) Ktze.-Gardiner's Island in rich woods.

Clinopodium vulgare L.-Gardiner's Island in rocky woods.

Collinsonia canadensis L.-Gardiner's Island in rich woods.

Glecoma hederacea L.-Rare at Orient.

Hedeoma pulegioides (L.) Pers.-Rare in shaded places.

Koellia flexuosa (Walt.) MacM.-Orient.

K. mutica (Mx.) Britton-Rare at Orient.

$K$. virginiana (L.) MacM.--Rare at Orient.

Lamium amplexicaule L.-Orient in cultivated fields.

Leonurus Cardiaca L.-Rare.

Lycopus americanus Muhl.

L. membranaceus Bicknell.

L. sessilifolius A. Gray-Southold.

L. uniflorus $\mathrm{Mx}$.

L. virginicus L.

Marrubium vulgare L.-Orient.

Melissa officinalis L.-Rare at Greenport.

Mentha gentilis L. - Rare at Orient along roads.

$M$. piperita L.-Rare at Orient.

$M$. spicata $\mathrm{L}$.

Nepeta Cataria L. 
Prunella vulgaris $\mathrm{L}$.

Scutellaria galericulata L.-Swamps.

S. lateriflora L.-Orient and Greenport in low woods.

Teucrium canadense L.-Sandy beaches and rocky woods; specimens in N. Y. State Herbarium.

Trichostema dichotomum L.-Plants with pink flowers (!); reported in N. Y. State Mus. Bull. I57:43. I9I2.

\section{SOLANACEAE}

Datura Stramonium L.-Gardiner's Island and elsewhere.

Lycium halimifolium Mill.-Rare.

Lycopersicon Lycopersicon (L.) Karst.-Escaped.

Physalis peruviana L.-Escaped at Orient in waste places and in gardens.

Physalodes physalodes (L.) Britton-Escaped at Orient.

Solanum Dulcamara L.-Rare.

S. nigrum L.-Rare on sandy beaches.

\section{SCROPHULARIACEAE}

Agalinis maritima Raf.-Orient on salt marshes; specimens in N. Y. State Herbarium. (Gerardia maritima Raf.)

A. pirpurea (L.) Britton-Orient; specimens in N. Y. State Herbarium.

A. tenuifolia (Vahl) Raf.-Dry hills.

Chelone glabra L.-Rare at Greenport.

Dasystoma flava (L.) Wood-Greenport.

D. pedicularia (L.) Benth.-East Marion.

D. virginica (L.) Britton.

Gratiola aurea Muh1.-Rare at Orient.

Iiysanthes aitenuata (Muhl.) Small-Orient.

I. dubia (L.) Barnh.-Orient.

Linaria canadensis (L.) Dum.

L. Linaria (L.) Karst.

Melampyrum lineare Lam.- The leaves are variable in outline.

Mimulus ringens $\mathrm{L}$.-Greenport.

Pedicularis canadensis L.-Orient.

Scrophularia leporella Bicknell-Rare at Orient.

Verbascum Blattaria L.

$V$. Thapsus L.

Veronica arvensis L.-Orient.

$V$. officinalis L.-East Marion.

$V$. peregrina $\mathrm{L}$.-Orient.

V. serpyllifolia L.

\section{LENTIBULARIACEAE}

Setiscapella cleistogama (A. Gray) Barnhart-Southold; determined at Bureau of Plant Industry. (Utricularia cleistogama (A. Gray) Britton.)

Stomoisia cornuta (Mx.) Raf.-Southold. (Utricularia cornuta Mx.)

\section{OROBANCHACEAE}

Lepiamnium virginianum (L.) Raf.-Greenport.

Thalesia uniflora (L.) Britton-Rare at Orient. 


\section{BIGNONIACEAE}

Bignonia radicans L.- Rarely escaped at Orient. (Tecoma radicans (L.) DC.)

\section{Phrymaceae}

Phryma Leplostachya L.-Gardiner's Island and Greenport in woods.

Plantago aristata $\mathrm{Mx}$.

\section{Plantaginaceae}

$P$. halophila Bicknell-Orient on salt marshes; plants earlier to flower and more downy than Plantage major L.

P. lanceolata $\mathrm{L}$.

$P$. major L.

$P$. maritima L.-Orient; specimens in N. Y. State Herbarium.

$P$. media L.

P. pusilla Pursh-Rare on dry hilltops; specimens in N. Y. State Herbarium.

P. Rugelii Dcne-East Marion, shores of a pond.

$P$. virginica L.-East Marion in sandy soil.

\section{Cephalanthus occidentalis L.}

\section{RUBIACEAE}

Galium Aparine L.-Orient in low rich shaded places.

G. circaezans Mx.-Rich woods.

G. Claytoni Mx.-Low grounds.

G. lanceolatum Torr.-Rare at Orient; determined at N. Y. Botanical Garden.

G. palusire L.-Greenport.

G. pilosum Ait.-Rare at Orient; the flowers purple.

G. tinctorium L.-Greenport in swampy woods.

G. triflorum Mx.--Greenport.

Mitchella repens L.-Plants sometimes having unusually large leaves.

\section{CAPRIFOLIACEAE}

Diervilla Diervilla (L.) MacM.

Lonicera japonica Thunb.--Orient and East Marion in woods.

L. sempervirens L.-East Marion.

Sambucus canadensis $\mathrm{L}$.

S. racemosa $\mathrm{L}$.- Rare at Orient, the station now destroyed; determined at Bureau of Plant Industry.

Triosteum aurantiacum Bicknell.

T. perfoliatum L.-Rare at Orient.

Viburnum acerifolium L.-More abundant at Greenport than at Orient.

$V$. dentatum L. - "A form with leaves decidedly acuminate"; reported in N. Y. State Mus. Bull, I50:49. I9II.

V. Lentago L.-Rare at Orient.

$V$. venosum Britton-Rare at East Marion in swamps; reported in N. Y. State Mus. Bull. I50: 4I. I9II.

\section{VALERIANACEAE}

Valeriana officinalis L.-Orient. 


\section{Campanulaceae}

Campanula rapunculoides L.-Rarely escaped at Orient. Specularia perfoliata (L.) A. DC.-Rare in hilly woods.

\section{LOBELIACEAE}

Lobelia cardinalis L.,-Rare from Greenport to Southold.

L. inflata $\mathrm{L}$.

L. syphilitica L.-Rare at Orient in low ground.

\section{Cichoriaceae}

Apargia nudicaulis (L.) Britton-Specimens in N. Y. State Herbarium, (Leontodon nudicaule (L.) Banks.)

Cichorium Intybus L.-Orient.

Crepis capillaris (L.) Wallr.-Orient. (Crepis virens L.)

C. setosa Hall. f.-Reported in N. Y. State Mus. Bull. I50: 29. I9II.

C. tectorum L.-Rare at Orient; determined by Dr. C. H. Peck.

Hieracium aurantiacum L.-Rare at Orient.

$\dot{H}$. Gronovii L.-Orient; specimens in N. Y. State Herbarium.

H. paniculatum L.-Greenport.

H. scabrum Mx.-Plants with more naked stems than usual; specimens in N. Y. State Herbarium.

H. venosum L.

Hypochaeris radicata L.-Orient; specimens in N. Y. State Herbarium.

Krigia virginica (L.) Willd.-Orient in light soil.

Lactuca canadensis L.-Cedar woods and elsewhere; also the var. montana Britton.

L. sagittifolia Ell.-Orient.

L. spicata (Lam.) Hitchc.

Lapsana communis L.-Rare at Orient; determined by P. L. Ricker.

Leontodon erythrospermum (Andrz.) Britton-Light soil and on sandy beaches at Orient. (Taraxicum erythrospermum Andrz.)

L. Taraxicum L.

Nabalus serpentarius (Pursh) Hook.-Greenport.

$N$. trifoliolatus Cass.

Picris echioides L.-Reported in N. Y. State Mus. Bull. I39: 28 . I9Io.

$P$. hieracioides L.-Rare at Orient; determined by Dr. C. H. Peck; reported in N. Y. State Mus. Bull. I50:37. I9II.

Sonchus arvensis L.-Rare at Orient.

S. asper (L.) Hill.-Orient.

S. oleraceus L.

\section{Ambrosiaceae}

Ambrosia elatior L.-(A mbrosia artemisiaefolia L.)

A. trifida L.-Rare at Orient in cultivated fields.

Xanthium commune Britton-Orient on beaches.

$X$. spinosum L.-Rare on Gardiner's Island.

\section{Compositae}

Achillea Millefolium L.-Also the forma rosea.

Anaphallis margaritacea (L.) Benth. \& Hook.-Rare. 
Antennaria neglecta Greene.

A. plantaginifolia (L.) Richards.

Anthemis arvensis L.-Rare at Orient in meadows and waste places.

A. Colula L.

A. tinctoria L.-Rare at Orient in meadows; specimens in N. Y. State Herbarium. Arctium minus Schkr.

Ariemisia caudata Mx.-Determined by Dr. C. H. Peck.

A. Stellariana Bess.-Orient on beaches.

Aster cordifolius L.-Greenport.

A. divaricatus $\mathrm{L}$. - Woods.

A. dumosus L.-Orient in sandy soil.

$A$, ericoides L.-Southold.

A. laevis L.-Low woods; also the long-leaved form.

A. lateriflorus (L.) Britton-Orient in woods.

A. macrophyllus L.-The plants are variable.

A. multiflorus Ait.

A. novae-angliae L.-More common on Gardiner's Island than at Orient.

A. novi-belgii L.-Low grounds.

A. paniculatus Lam.

A. patens Ait.-Dry hills.

A. puniceus $\mathrm{L}$.

A. salicifolius Lam.-Orient in low places.

A. spectabilis Ait.-Orient; also a form with white flowers.

A. subulatus $\mathrm{Mx}$.

A. tenuifolius L.

A. Tradescanti L.

A. undulatus $\mathrm{L}$.

A. vimineus Lam.

Baccharis halimifolia L.-Orient about salt marshes.

Bidens cernua L.

B. comosa (A. Gray) Wiegand-Low grounds.

B. connata Muhl.--Low grounds; also a form with entire leaves.

B. discoidea (T. \& G.) Britton.

B. frondosa $\mathrm{L}$.

B. laevis (L.) BSP.

Centaurea Cyanus L.-Rare at Orient.

C. Jacea L.-Rare at Orient in a dry pasture.

Chrysanthemum Leucanthemum $\mathrm{L}$.

Chrysopsis falcata (Pursh) Ell.--Orient and Southold.

C. mariana (L.) Ell.

Cirsium arvense (L.) Scop.

C. discolor (Muhl.) Spreng.

C. horridulum Mx.-Specimens in N. Y. State Herbarium. (Cirsium spinosissimum (Walt.) Scop.)

C. lanceolatum (L.) Hill.-Moist places.

C. muticum $\mathrm{Mx}$.- Rare at Orient.

C. odoratum (Muhl.) Britton.

Doellingeria umbellata (Mill.) Nees. 
Erechtites hieracifolia (L.) Raf.-Two forms occur, hairy and smooth plants; specimens in N. Y. State Herbarium.

Erigeron annuus (L.) Pers.

E. philadelphicus L.

E. pulchellus $\mathrm{Mx}$.

E. ramosus (Walt.) BSP.

Eupatorium aromaticum L.-Southold.

E. hyssopifolium L.-Southold.

E. maculatum L.-Orient in low grounds.

E. perfoliatum L.

E. purpureum L.

E. verbenaefolium $\mathrm{Mx}$.

Euthamia graminifolia (L.) Nutt.

E. tenuifolia (Pursh) Greene-Southold.

Galinsoga parviflora Cav.-Orient along roads; the var. hispida DC.

Gnaphalium obtusifolium L.

G. uliginosum L.

Helianthus annuus L.-Rarely escaped at Orient.

$H$, divaricatus $\mathrm{L}$.

H. giganteus $\mathrm{L}$.

H. strumosus L.

H. tuberosus L.

Lacinaria spicata (L.) Ktze.-Orient, a colony of 50 or more plants; specimens in N. Y. State Herbarium.

Leptilon canadense (L.) Britton.

Mariana mariana (L.) Hill-Orient in a garden; specimens in N. Y. State Herbarium.

Onopordum Acanthium L.-More common on Gardiner's Island than at Orient and East Marion; specimens in N. Y. State Herbarium.

Pulchea camphorata (L.) DC.

Rudbeckia hirta L.

Senecio aureus L.-Rare at Orient.

$S$. vulgaris L.-Rare in cultivated fields.

Seriocarpus asteroides (L.) BSP.

Solidago altissima $\mathrm{L}$.

S. aspera Ait.-Reported in N. Y. State Mus. Bull. I39: 30. I9I0.

S. bicolor L.

S. caesia L.

S. juncea Ait.-Plants variable.

S. nemoralis Ait.

S. odora Ait.

S. rugosa Mill.-The broad-leaved form and the form with small leaves.

S. sempervirens L.

S. serotina Ait.—Orient in swamps.

S. ulmifolia Muhl.

Tanacetum vulgare $\mathrm{L}$.

Vernonia noveboracensis (L.) Willd.-Orient. 


Reprinted without change of paging from TORREYA, a m:onthly journal of botanical notes and news, published by the Torrey Botanical Club. Price $\$ 1.00$ per year. Matter for publication should be sent to Norman Taylor, Brooklyn Botanic Garden, Brooklyn, N. Y. 
[Reprinted from TorreyA, Vol. 17, No. 7, July, 1917 ]

\section{THE FLORA OF THE TOWN OF SOUTHOLD, LONG ISLAND AND GARDINER'S ISLAND}

By Stewart H. Burnham and Roy A. Latham

\section{First Supplementary List}

The preliminary flora was published in Torreya I4: 20 I-225. Nov. I9I4 and 229-254. Dec. I9I4. The majority of the enumerated plants were collected in I9I5. Mr. Frank Dobbin of Shushan, N. Y., also visited Orient Aug. IO-I5, I9I5, and spent considerable of the time collecting.

The territory of the region included in this flora lies wholly in the glaciated region. Along the shore of Long Island Sound is the obscure inner moraine of the Wisconsin ice sheet; and from this moraine is an outwash of thin deposits forming sandy plains over the older Pleistocene formation which shows through and in places controls the topography. Some of the beaches and many of the swamps and marshes belong to the Recent epoch. Gardiner's Island lies between the inner and outer moraines of the Wisconsin ice sheet: and being of more rugged topography "seems to have encouraged a more extensive reworking of the" older Pleistocene "deposits by the Wisconsin ice and a greater deposition of" the till sheet or ground moraine. ("The Geology of Long Island," by Myron L. Fuller, U. S. Geol. Survey Professional Paper 82: Washington. I9I4.)

The authors are greatly indebted to many specialists, who have made it possible to publish the following catalogue of species.

\section{InSECt Galls*}

Andricus cornigerus O. S.-Horned Knot Oak Gall.

Asphondylia globosus O. S.--On stems of Helianthus divaricatus.

* The majority of these galls were named by Dr. E. P. Felt, state entomologist of the State of New York. 
Diastrophus Potentillae Bass.-Cinquefoil Axil Gall; on stems of Potentilla canadensis.

Rhopalomyia Solidaginis Loew-Goldenrod Bunch Gall.

Trypeta Solidaginis Fitch-Goldenrod Ball Gall.

\section{Thallophyta}

\section{EUTHALLOPHYTA}

\section{EUPHyCEAE*}

Botrydium granulatum (L.) Grev.-On wet earth.

Griffithsia tenuis Ag.--Long Island Sound.

Polysiphonia violacea (Roth) Grev.-On rocks in the Sound.

Ralfsia verrucosa (Aresch.) J. Ag.-On rocks in shallow water.

Rhizoclonium hieroglyphicum (Ag.) Kütz.-About roots of bushes in a fresh water swamp.

Rivularia atra Roth-On rocks at the water's edge.

Ulothrix implexa Kütz.--On rocks at mid-tide mark, Orient bay.

\section{FUNGI}

\section{SCHIZOMYCETES}

Bacillus iracheiphilus Erw. Smith-On Cucumis sativus; determined by Mr. F. V. Rand.

\section{EUMYCETES}

Phytophthora Phaseoli Thaxt.-On Phaseolus lunatus; determined by Mr. Rand. Plasmopara cubensis (B. \& C.) Humphrey-On Cucumis sativus; determined by Mr. Rand.

\section{Ascomycetes (Excluding Pyrenomycetes)}

Chlorosplenium chlora (Schw.) Mass.-On decayed wood of Quercus coccinea; determined by Dr. F. J. Seaver.

Dasyscypha Ellisiana (Rehm) Sacc.-On bark of living Pinus rigida; determined by Dr. Seaver.

Lecanidion atritum (Hedw.) Rabenh.-On bare wood of Toxylon pomiferum; determined by Dr. C. E. Fairman.

Melittosporium hysterinum (Fr.) Gill.-On bare wood of Juniperus virginiana; determined by Dr. Fairman.

Pseudopeziza Medicaginis (Lib.) Sacc.-On leaves of Medicago sativa; determined by Dr. Fairman.

Taphrina Quercus (Cke.) Sacc.-On leaves of Quercus velutina; determined by Dr. H. D. House.

\section{Ascomycetes (PYRENOMYCETES) $\dagger$}

Anthostomella sepelibilis (B. \& C.) Sacc.-On old stems of Smilax rotundifolia.

Botryosphaeria Ribis Grossen. \& Duggar-On old stems of cultivated Grossularia.

* The algae were determined by Dr. M. A. Howe and are preserved in the Herbarium of the New York Botanical Garden.

† Unless otherwise stated, the Pyrenomycetes were determined by Dr. C. E. Fairman. 
Cucurbitaria elongata (Fr.) Grev.-On old twigs of Robinia Pseudo-Acacia; also the macropycnidial stage, Hendersonia Robiniae West: determined by Dr. House. Diaporthe ocularia (C. \& E.) Sacc.-On twigs and branches of Ilex verticillata.

D. rhoina (C. \& E.) E. \& E.-On twigs and branches of Rhus copallina.

Diatrypella Cephalanthi (Schw.) Sacc.-On twigs of Cephalanthus occidentalis.

Dothidea ribesia (Pers.) Fr.-On old stems of cultivated Grossularia.

Eutypella deusta (E. \& E.) E. \& E.-On old wood of oak; determined by Dr. House.

E. prunastri (Pers.) Sacc.-On twigs and branches of Padus virginiana.

Fimetaria fimicola (Roberge) Griffiths \& Seaver-On old paper.

Gloniopsis Cookeana (Ger.) Sacc.-On sumac, Myrica carolinensis and Xolisma ligustrina; determined by Dr. House.

G. fibriseda (Ger.) Sacc.-On twigs of Acer rubrum.

G. Lonicerae (Phill. \& Hark.) Berl. \& Vogl.-On twigs of Lonicera japonica.

Hypoderma Smilacis (Schw.) Rehm-On dead stems of Smilax rotundifolia.

Hypoxylon cohaerens (Pers.) Fr.-On branches of Fagus grandiflora at Greenport.

H. smilacicolum Howe-On stems of Smilax rotundifolia at Greenport.

Hysteriographium Vaccinii (Schw.) Fairman, n. comb.-On twigs of Vaccinium atrococcum at Greenport. In Saccardo's Sylloge Fungorum this is described as Hysterium Vaccinii Schw.

H. vulvatum (Schw.) Sacc.-On Qvercus velutina; determined by Dr. House.

Laestadia polystigma (E. \& E.) Sacc.-On leaves of Quercus velutina.

Lophiotrema praemorsum (Lasch) Sacc.-On old stems of Brassica oleracea gemmifera (Brussels sprouts).

Lophodermium arundinaceum (Schrad.) Chev.-On Ammophila arenaria. Dr. Fairman says, "I have found $L$. arundinaceum in America on grain stems but have no specimens on Ammophila: and have no knowledge of its ever having been found here on this host."

Mazzantia sepium Sacc. \& Penz.-On stems of Convolvulus sepium. Dr. Fairman says, "I have never found it in America; although I have specimens from London, Canada."

Myiocopron Smilacis (DeNot.) Sacc.-On twigs of Smilax rotundifolia at Greenport.

Nummularia microplaca (B. \& C.) Cke.-On dead branches of Sassafras Sassafras. Dr. Fairman says, "Reprinted in this country from the South, Ohio and Virginia."

Phyllachora Cyperi Rehm-On Cyperus esculentus; determined by Dr. House.

Physalospora Potentillae Rostr.-On stems and galls of Potentilla canadensis. Dr. Fairman says the type of this fungus was found on Potentilla maculata in Greenland: and that "it has not been found in this country by anyone before so far as I know. The best specimens of your collection are on the galls," Diastrophus Potentillae.

Pleospora herbarum (Pers.) Rabenh.-On stems of Allium Cepa, Asparagus offinalis, Vagnera stellata, Moehringia lateriflora, Silene caroliniana and Glaucium Glaucium.

P. Salsolae Fckl.-On stems of Salicornia ambigua and Salsola Kali. Dr. Fairman says, "I have never had it from this country: but Ellis and Everhart list it on Salicornia herbacea, California (Harkness)."

Rhytisma Ilicis-canadensis Schw.-On leaves of Ilex verticillata at Southold; determined by Dr. House. 
Valsa Liquidambaris Schw.-On branches of Hamamelis virginiana; determined by Dr. House.

V. pauperata C. \& E.-On twigs and branches of Acer rubrum at Greenport.

\section{HYPOMYCETES}

Cercospora Teucrii E. \& K.- "Orient Point, on living leaves of Teucrium canadense." N. Y. State Mus. Bull. I79: 26. I9I5.

Cladosporium Typhae Schw.-On old leaves and stems of Typha latifolia; determined by Dr. Fairman.

Trichoderma lignorum (Tode) Harz.--On bare wood of Quercus velulina; determined by Dr. Fairman.

\section{Melanconiales}

Pestalozzia conigena Lev.-On cones of Thuja occidentalis; determined by Dr. Fairman.

\section{SPHAEROPSIDEAE*}

Cytospora leucostoma (Pers.) Sacc.-On twigs of Amygdalus Persica.

Diplodia hyalospora C. \& E.-On old stems of Chenopodium album.

D. Maydis (Berk.) Sacc.-On old stalks of Zea Mays.

Diplodina Alriplicis Vestgr.- On old stems and withered leaves of Atriplex hastata. Dr. Fairman says when this fungus occurs on stems, it is called Diplodina Atriplicis; when on leaves, Ascochyta Atriplicis Died. On your plants "we have fungi on both, so that it is as you please what you say, Diplodina or Ascochyta, at present. I have referred yours to Diplodina because most prominent on the stems."

Labrella nitida Schw.-On stems of Polygonatum commutatum.

Leptostroma flicinum Fr.-On old stipes of Athyrium Filix-foemina.

L. virgultorum Sacc.-On stems of Aralia nudicaulis.

Leptostromella hysterioides (Fr.) Sacc.-Determined by Dr. House.

Leptothyrium litigiosum (Desm.) Sacc.-On Osmunda cinnamomea; determined by Dr. House.

L. Pomi (Mont. \& Fr.) Sacc.-On the skin of the fruit of Malus Malus.

Macrophoma pulchrispora (Pk. \& Clint.) Sacc.-On stems of Persicaria pennsylvanica.

Phlyctaena arcuata Berk.-On dead stems of Arctium minus.

P. complanata (B. \& C.) Sacc.-On dead stems of Tiniaria Convolvulus.

Phoma Cydoniae Sacc. \& Schulz.-On old fruit of Cydonia vulgaris (Quince).

$P$. longipes B. \& C._- Orient Point on Morus alba." The plant reported in the preliminary list to Phoma moricola Sacc. should be referred to this species. N. Y. State Mus. Bull. I88: 37. I916.

P. media E. \& E.-On old stems and branches of Aspardgus officinalis.

$P$. nebulosa (Pers.) Sacc.-On dead stems of Lepidium virginicum.

$P$. sepincola (Kickx.) Sacc.-On branches of rambler rose.

$P$. strobiligena Desm.-On cones of Thuja occidentalis.

$P$. verbascicola (Schw.) Cke.-On stems of Verbascum Thapsus.

Phomopsis cryptica (Nits.) Trav.-On twigs of Lonicera japonica.

* Unless otherwise stated, the Sphaeropsideae were determined by Dr. C. E. Fairman. 
P. occidentalis Sacc., var. irregularis Trav.-On twigs and branches of Gleditschıa iriacanthos.

P. vepris (Nits.) Trav.-On stems of Rubus procumbens.

Phyllosticta Baccharidis Dearness \& House-" "On living leaves of Baccharis halimifolia, Orient Point." This species is described in N. Y. State Mus. Bull. I79: 29. I9I5: and the type is in the herbarium of the N. Y. State Museum.

$P$. orobella Sacc.- "On languishing leaves of Lathyrus maritimus, Orient Point, New to America." N. Y. State Mus. Bull. I79: 30. I9I5.

Rhabdospora Lonicerae (C. \& E.) Sacc.--On dead twigs of Lonicera japonica. Dr. Fairman says, "a rare find. It was originally found by Ellis on Lonicera in New Jersey and sent by him to Cooke who called it Cryptosporium Lonicerae C. \& E. in Grevillea 6: 83. March I878. It has curved hyaline spores and really seems to be a good Cryptosporium. I do not think Ellis ever found it again."

$R$. subgrisea $\mathrm{Pk}$.-On stems of Solidago sempervirens; determined by Dr. House.

Septoria Caryophylii Scalia-On leaves of Dianthus caryophyllus. Dr. Fairman says, "I presume this has been called S. Dianthi: but it agrees better with the above."

S. graminum Desm.-On leaves of Dactylis glomerata; determined by Dr. House. Sphaeronaema acerinum $\mathrm{Pk}$. - On dead bark and twigs of Acer rubrum.

Sphaeropsis Arctostaphylii (Vize) Sacc.-On bare wood.

S. Celastrina Pk.-On Celastrus scandens.

S. Juniperi $\mathrm{Pk}$.- On Juniperus virginiana; determined by Dr. House.

S. rubicola C. \& E.-On stems of Rubus procumbens.

S. sepulta E. \& E.-On dead twigs of Morus alba at Orient Point. N. Y. State Mus. Bull. I88: 53. I9I6.

Vermicularia petiolicola P. Brun-On petioles of Geranium maculatum.

\section{BASIDIOMYCETES}

\section{USTILAGINACEAE}

Urocystis Cepulae Frost-On Allium Cepa; determined by Dr. G. P. Clinton.

Ustilago Crus-galli Tracy \& Earle-On Echinochloa Crus-galli; determined by Dr. Clinton.

U. Rabenhorstiana Kühn-On Syntherisma sanguinale; determined by Dr. Clinton.

\section{MelampsoraceaE*}

Melampsora Medusae Thüm.-On leaves of Populus tremuloides.

\section{COLEOSPORIACEAE}

Coleosporium delicatulum (A. \& K.) H. \& L.-Southold on leaves of Euthamia tenuifolia.

C. Helianthi (Schw.) Arth.-On leaves of Helianthus divaricatus.

* Unless otherwise stated the Rusts were determined by Dr. J. C. Arthur and are preserved in the Herbarium of Dr. Arthur at Purdue University, Lafayette, Indiana. The authors are indebted to Dr. Arthur, who has read the manuscript of the Rusts. 


\section{PuCciniaceat}

Gymnosporangium globosum Farl.-Greenport on Crataegus chrysocarpa.

Kuehneola Uredinis (Lk.) Arth.-On leaves of Rubus alleghaniensis.

Phragmidium americanum Diet.-On leaves of Rosa blanda.

P. Potentillae-canadensis Diet.-On leaves of Potentilla canadensis.

$P$. Rosae-setigerae Diet.-On leaves of Rosa carolina.

Polythelis Thalictri (Chev.) Arth.-On leaves of Thalictrum revolutum. (Puccinia Thalictri Chev.)

Puccinia Acetosae (Schum.) Körn.-On leaves of Rumex Acetosella. Dr. Arthur says, "this rust has been found at Woods Hole, Massachusetts, in South Carolina and Florida. Your locality making the fourth one."

P. angustata Pk.--Southold and Greenport on Scirpus cyperinus and S. pedicillatus.

$P$. canaliculata (Schw.) Lagerh.-On Cyperus esculentus, the telial stage; determined by Dr. House.

P. Caricis-strictae Diet.-Southold on Carex stricta, the amphisporal stage.

P. Cichorii (DC.) Bell-On leaves of Cichorium Intybus.

P. Clematidis (DC.) Lagerh.-On leaves of Agropyron repens and Hordeum sativum. (Puccinia Agropyri E. \& E.; P. agropyrina Erikss.)

$P$. Convolvuli (Pers.) Cast.-On leaves of Convolvulus sepium.

P. Eleocharidis Arth.-On Eleocharis tenuis.

P. epiphylla (L.) Wettst.-On Poa pratensis. (Puccinia poarum Niessl.)

$P$. extensicola Plowr.-On Carex hormathodes, C. scoparia, C. straminea, C. vulpinoidea and Dulichium arundinaceum. (Puccinia Dulichii Sydow; P. vulpinoidis Diet. \& Holw.)

P. fraxinata (Lk.) Arth.-On Spartina patens. Dr. Arthur says we have this rust "on the same host from Delaware and New Jersey: but not before from any point in New York."

P. Grossulariae (Schum.) Lagerh.-Greenport on Carex debilis, (Puccinia uniporula Orton.)

P. Impatientis (Schw.) Arth.-Gardiner's Island on Agrostis alba and Elymus striatus. (Puccinia perminuta Arth.)

P. Phlei-pratensis Erikss. \& Henn.-On Phleum pratense.

P. poculiformis (Jacq.) Wettst.-On Agrostis alba and Dactylis glomerata.

Puccinia Polygoni-amphibii Pers.-On leaves of Persicaria pennsylvanica, $P$. punctata, Tiniaria scandens and Tovara virginiana.

P. Prenanthis-racemosae Sydow-Greenport on leaves of Nabalus trifoliolatus.

P. Proserpinaceae Farl.-Greenport on leaves of Proserpinaca palustris. Dr. Arthur says, "known only from Massachusetts, Illinois and Wisconsin."

P. Rhamni (Pers.) Wettst.-On Avena sativa.

$P$. Smilacis Schw.-Sonthold on Smilax glauca.

$P$. Xanthii Schw.-On Xanthium commune.

Uredinopsis mirabilis (Pk.) Magn.-Gardiner's Island on Onoclea sen ibulis.

Uromyces fallens (Desmaz.) Kern-On Trifolium pratense. (Nigredo fallens (Desmaz.) Arth.)

U. Hyperici-frondosi (Schw.) Arth.-Greenport on Triadenum virginicum. (Nigredo Hyperici-frondosi (Schw.) Arth.)

U. Junci-effusi Sydow-Greenport on Juncus effusus. (Nigredo Junci-effusi (Sydow) Arth.) 
U. minutus Diet.-On Carex virescens. (Nigredo minuta (Diet.) Arth.)

$U$. pedatatus (Schw.) J. Sheldon-Southold on Andropogon virginicus. (Nigredo pedatata (Schw.) Arth.)

$U$. perigynius Halst.-Greenport on Carex intumescens, (Nigredo perigynia Halst.) Arth.

U. Polygoni (Pers.) Fckl.-On leaves of Polygonum aviculare. (Nigredo Polygoni (Pers.) Arth.)

U. Scirpi (Cast.) Burr.-On Scirpus robustus.

U. Trifolii (Hedw. f.) Lev.-On leaves of Trifolium hybridum and T. repens. (Nigredo Trifolii (Hedw. f.) Arth.)

U. uniporulus Kern-On Carex virescens. (Nigredo uniporula (Kern) Arth.)

\section{TREMELLACEAE}

Ulocolla foliacea (Pers.) Bref.-On dead bark of Quercus velutina; determined by Dr. C. G. Lloyd.

\section{DACRYOMYCETACEAE}

Dacryomyces deliquescens (Bull.) Duby-On rotten wood of oak; determined by Dr. Lloyd.

\section{THELEPHORACEAE}

Corticium incarnatum (Pers.) Fr.-On branches of Sambucus canadensis; determined by Dr. E. A. Burt.

Stereum albo-badium Schw.-On old stems of Brassica oleracea gemmifera (Brussels sprouts); determined by Dr. Burt, who says, "a species I have seen heretofore on woody stems only."

S. fasciatum Schw.-Greenport on dead trunk of Quercus velutina; determined by Dr. Lloyd. Stereum versicolor Fr. previously listed belongs here.

Tremellodendron pallidum (Schw.) Burt-On earth in low woods at Greenport; determined by Dr. Lloyd. (Thelephora Schweinitzii Pk.)

\section{HYDNACEAE}

Hydnum imbricatum L.-Moist soil in woods at Greenport; determined by Dr。 Lloyd.

\section{POLYPORACEAE*}

Boletus Frostii Russell-Rare in rich earth in open woods, Greenport and Gardiner's Island.

Cyclomyces Greeni Berk.-On earth in rich woods, Greenport.

Merulius Corium (Pers.) Fr.-On old bark of Myrica carolinensis.

Polyporus adustus (Willd.) Fr.-On old wood of Quercus velutina at Greenport.

$P$. albellus $\mathrm{Pk}$. - On old wood.

P. amygdalinus Berk.-Greenport on stump of Quercus velutina. Dr. Lloyd says, "the second specimen I have seen, a very interesting find."

$P$. dichrous Fr.-On stumps of Juniperus virginiana.

* Except the Boletus, the Polypores were determined by Dr. C. G. Lloyd and are preserved in the Herbarium of the Lloyd Museum and Library, Cincinnati, Ohio. 
P. squamosus (Huds.) Fr.-On living trunk of Salix nigra, Gardiner's Island. Polystictus cinnamomeus (Jacq.) Sacc.-Rich soil in oak woods at Greenport. Poria medulae-panus (Pers.) Fr.-On spruce timber in a cellar.

$P$. pinea Pk.-On old log of Pinus Strobus.

$P$. radula (Pers.) Fr.-On Quercus velutina and rotten wood of Sassafras Sassafras.

\section{AgARICACEAE}

Panus levis Berk.-On oak wood in a shed; determined by Dr. Lloyd, who says, "this is an American plant that is very rarely received by me. The spores of Panus levis are 4-6 X I0-1 $2 \mu$ and slightly arcuate."

Pleurotus striatulus Fr.-On old wood; determined by Dr. Lloyd.

\section{GASTEROMYCETES}

Calastoma circumscissum (B. \& C.) Morg.- Sandy soil in open cedar woods; determined by Dr. Lloyd.

Lycoperdon umbrinum Pers.-Sandy soil in open woods; determined by Dr. Lloyd. (Lycoperdon glabellum Pk.)

Sphaerobolus stellatus Tode-On old wood of Vitis bicolor; determined by Dr. Fairman.

\section{LICHENES*}

Biatora rivulosa (Ach.) Fr.-On bark of oak at Greenport.

B. uliginosa (Schrad.) Fr.-In open places on light bare soil.

Cladonia macilenta styracella (Ach.) Wainio-On old rotten pine log in sandy woods. C. ochrochlora ceratodes Fkl.-On sandy soil in open woods.

Cyrtidula rhoica Minks-On bark of sumac.

Lecanora ( $\$$ Ochrolechia) pallescens (L.) Schaer.-On bark of large oak trees in woods at Greenport.

Lobularia pulmonaria (L.) Hoffm.-On trunks of trees in woods at Greenport. (Sticta pulmonaria (L.) Ach.)

Peltigera polydactyla (Neck.) Hoffm.-About mossy roots of trees in moist woods at Greenport.

$P$. scutata (Dicks.) Leight.-Mossy banks in woods at Greenport; determined by Miss Mary F. Miller.

Physcia obscura virella (Ach.) Leight.-On bark of oak in woods at Greenport.

\section{HEPATICAE†}

Asterella tenella (L.) Bv.-On heavy soil along roadside in cedar woods.

Cephalozia fuitans (Nees) Spruce-Southold about base of trees in a sandy swamp; determined by Dr. A. W. Evans.

C. Francisci (Hook.) Dum.-On clean moist sand, at edge of a cranberry bog at the lake on Horton Point. Southold, forming beautiful green carpets $6 \mathrm{x}$ Io feet; determined by Dr. G. H. Conklin and Dr. Evans. Dr. Conklin says this species "has been found only a few times in North America. This the fourth or fifth

* Unless otherwise stated, the Lichens were determined by Mr. G. K. Merrill, Rockland, Maine.

† Unless otherwise stated, the Hepatics were determined by Dr. G. H. Conklin, Superior, Wisconsin: and are preserved in the Hepatic Herbarium of The Sullivant Moss Society. 
time." Dr. Evans in his "Notes on North American Hepaticae. VI" in Bryol. I8: 83. Sept. I9I5 says that the geographical distribution of Cephalozia Francisci "in North America is so incompletely known that the report of the following new stations seem justifiable." A station on Cape Breton Island, Nova Scotia: and "Southold and Orient Point, Long Island, New York, R. Latham," are reported. "The last two stations which represents a marked extension of the known range to the southward," having been found previously in Maine and New Hampshire," are of especial interest and indicate that the plant ought to be looked for in eastern Connecticut and Rhode Island.". This rare hepatic has only been found at Horton Point, Southold: but has never been found at Orient Point, as stated above!

C. macrostachya Kaal.- Southold about base of bushes in a sandy swamp; determined by Dr. Evans.

C. media Lindb.-On old logs in moist woods at Greenport.

Fossombronia foveolata Lindb.-Sandy swamps, Horton Point; and dry soil in cedar woods, Orient, fruiting in November; determined by Dr. Evans and Dr. Conklin.

Lophocolea minor Nees-On a mossy rock in a swamp.

Notothylas orbicularis (Schw.) Sull.-Muddy bottom of a pasture pond at Orient. Odontoschisma Sphagni (Dicks.) Dumort.-Edge of woodland swamp at Orient; determined by Miss Annie Lorenz.

Pallavicinia Lyellii (Hook.) S. F. Gray-About mossy base of trees in moist woods and swamps, Greenport and Southold.

Pellia Fabroniana Raddi-Edge of stream in woods at Greenport. Dr. Conklin says "this is a rare species."

Riccardia pinguis (L.) S. F. Gray-Among rushes on wet sandy shore of lake at Horton Point: and among grasses in brackish marsh at Orient; determined by Dr. Conklin and Dr. Evans.

\section{MUSCI}

Amblystegium Kochii B. \& S.- On old leather and wood in a shady place; determined by Dr. A. J. Grout.

Fissidens minutulus Sull.--Small pieces of sandstone, under a shady bank, edge of lake at Horton Point; determined by Mr. G. B. Kaiser.

Fontinalis dalecarlica B. \& S.-Trunks of bushes in wet places; determined by Mr. Kaiser.

Hypnum curvifolium Hedw.-All traces of this moss has disappeared where it was found in December I909. This species should probably be referred to Hypnum imponens Hedw.

Plagiothecium Roeseanum (Hampe) B. \& S.-Wet shady place; determined by Dr. Grout.

Pogonatum brevicaule (Brid.) Bv.-Wet stream bank at Greenport; determined by Mr. Kaiser.

Polytrichum commune uliginosum Hueb.-Dry soil at Southold; determined by Mr. Kaiser.

Sphagnum compactum DC.-Sandy bog at Southold; determined by Dr. A. L. Andrews.

S. subsecundum Nees-The form called $S$. inundatum Russ. in a sandy bog at Southold; determined by Dr. Andrews. 


\section{Pteridophyta}

Polypodiaceae

Dryopteris hexagonoptera (Mx.) C. Chr.-Moist woods, Gardiner's Island.

\section{LYCOPODIACEAE}

Lycopodium adpressum (Chapm.) Lloyd \& Underw.-Orient in a brackish meadow; the first club-moss found at Orient. No Lycopodiums have been found on Gardiner's Island.

\section{ANGIOSPERMAE}

\section{MONOCOTYLEDONES}

\section{ZANNICHELLIACEAE}

Potamogeton diversifolius Raf.-In the lake on Horton Point.

Zannichellia palustris L.-Shallow brackish stream, Gardiner's Island.

\section{Gramineae}

Agrostis alba L.- The var. aristata Gray, collected by Mr. Frank Dobbin in woods at Greenport; determined by Mrs. Agnes Chase.

Andropogon virginicus L.-Moist sandy soil, Southold; determined by Mrs. Chase. Elymus halophilus Bicknell-Salt marshes. The very light glaucous green plants grow in tufts: and are never as tall as the other wild ryes.

E. striatus Willd.-Rocky woods, Gardiner's Island; determined by Mr. Dobbin. Lolium multiflorum Lam.-Waste and cultivated grounds, rare at Orient; determined by Mrs. Chase.

Panicum flexile (Gattinger) Scribn.-Dry sandy soil, Southold; determined by Mrs. Chase.

P. Lindheimeri Nash-Dry ground, Mattituck; determined by Mrs. Chase.

$P$. virgatum L. - The var. cubense Griseb., collected by Mr. Dobbin at Orient; determined by Mrs. Chase.

Paspalum pubescens Muhl.-Dry pastures and cultivated fields; determined by Mrs. Chase.

Syntherisma sanguinalis (L.) Dulac-Common in cultivated fields and waste places; often known by the name of "Flat-grass."

\section{Cyperaceae}

Carex debilis Mx.-Greenport, the host of Puccinia Grossulariae.

C. laxiflora Lam.-Woodlands.

Cyperus Grayii Torr.-Abundant on the sands at Horton Point; also in sandy woods at Mattituck. "Orient Point": in the State Herbarium are two specimens collected on Long Island many years ago. "New Jersey is usually given as the northern range of this species." N. Y. State Mus. Bull. I76: 44. I9I5.

C. Houghtoni Torr.-The specimens, previously reported from Orient, are probably referable to Cyperus Grayii Torr.

\section{ORCHIDACEAE}

Blephariglottis psycodes (L.) Rydb.-Rich woods, Gardiner's Island; determined by Mr. Dobbin. 
Gymnadeniopsis clavellata (Mx.) Rydb.-Moist woods, Gardiner's Island; determined by Mr. Dobbin.

Ibidium ochroleucum (Rydb.) House-Dry hillsides, East Marion. The leaves are often half an inch wide: and the flowers, cream-colored and very fragrant. This species has been combined with Ibidium cernuum (L.) House: but the East Marion plants and those found in the Flora of the Lake George region, New York, appear quite distinct.

Liparis liliffolia (L.) L. C. Rich.-Swampy wood at Orient, very rare; collected by Mr. Vinton Richard.

Pogonia ophioglossoides (L.) Ker-In a brackish meadow at Orient, rare.

\section{DICOTYLEDONES}

\section{SALtCACEAE .}

Salix caprea L.-The var. pendula Hort. "Roadsides, Orient Point." N. Y, State Mus. Bull. I76: 44. I9I5.

\section{ULMACEAE}

Celtis occidentalis L.-Mr. Dobbin says he saw, Aug. I5, I9I5 on Gardiner's Island. trees with trunks two feet in diameter.

\section{MORACEAE}

Morus rubra L.-Two trees, edge of dry woods, near an old pathway, Gardiner's Island; probably introduced; determined by Mr. Dobbin.

\section{URTICACEAE}

Urtica urens L.-Rare in low waste ground, Gardiner's Island.

\section{Polygonaceae}

Fagopyrum Fagopyrum (L.) Karst.-Persisting in cultivated ground and waste places.

\section{Chenopodiaceae}

Dondia linearis (Ell.) Heller-Salt marshes and beaches.

\section{CORRIGIOLACEAE}

Scleranthus annums L.-Dry roadside, Greenport.

\section{SAXIFRAGACEAE}

Chrysosplenium americanum Schwein.-Along a muddy ditch in woods, Gardiner's Island; determined by Mr. Dobbin.

\section{Malaceae}

Pyrus communis L.-Woods and hedges.

\section{FABACEAE}

Meibomia canescens (L.) Ktze.-Edge of dry woods, Gardiner's Island. Melilotus officinalis (L.) Lam.-Rare in fields at Orient.

Vicia villosa Roth-Occasional in fields and waste places.

\section{Gerantaceae}

Geranium carolinianum L.-Orient in a sandy pasture. G. molle L.--Rare in a sandy pasture at Orient. 


\section{POLYGALACEAE}

Polygala ambigua Nutt.-Dry pastures, Orient and Gardiner's Island.

ELATINACEAE

Elatine americana (Pursh) Arn.-Shallow water of lake at Horton Point; determined by Mr. Percy Wilson.

\section{VACCINIACEAE}

Vaccinium vicinum Bicknell-In woods about a spring, Gardiner's Island, fruiting specimens Aug. I5, 1915. These specimens have been compared with the type in the Herbarium of New York Botanical Garden.

\section{Asclepiadaceae}

Asclepias purpurascens L.-Moist place in woods, Gardiner's Island.

\section{BORAGINACEAE}

Lappula virginiana (L.) Greene-Rich woods, Gardiner's Island; determined by Mr. Dobbin.

Myosotis laxa Lehm.-Rare on muddy shores, Gardiner's Island; determined by Mr. Dobbin.

\section{LABIATAE}

Teucrium littorale Bicknell-Wet woods, shores and salt marshes, Gardiner's Island and Orient.

\section{SOLANACEAE}

Datura Tatula L.-More common at Orient than the white-flowered species, Datura Stramonium L., with which $D$. Tatula has been combined; collected by Mr. Dobbin.

\section{LeNTibulariaceaE}

Utricularia geminiscapa Benj.- Shallow water of lake at Horton Point; determined by Dr. J. H. Barnhart. (Utricularia cleistogama (Gray) Britton:)

\section{Rubiaceae}

Diodia teres Walt.-Moist pasture at Orient.

\section{Ambrosiaceae}

Iva frutescens L.-Salt marshes at Orient; determined by Mr. Dobbin.

\section{Compositae}

Gnaphalium purpureum L.--Rare in dry woods.

Helianthus decapetalus L.-Moist woods at Orient; leaves a little narrower than usual and in whorls of four below.

Ionactis linariifolius (L.) Greene-Sandy soil on Long Beach, Orient, rare.

Mikania scandens (L.) Willd.-Edge of wet woods, Gardiner's Island; determined in part by Mr. Dobbin.

Solidago speciosa Nutt.- Rare in dry woods at Orient. The specimen had entire leaves with ciliolate margins. 


\title{
THE FLORA OF THE TOWN OF SOUTHOLD, LONG ISLAND AND GARDINER'S ISLAND
}

\author{
By Stewart H. Burnham and Roy A. Latham
}

\section{Second Supplementary List*}

The following local observers have assisted in this list, by collecting or reporting unusual species; therefore establishing many new records and also new stations for species already reported in the two preceding lists. Mrs. F. R. Mitchell of Southold is specially mentioned for kindness in allowing a study of her long and interesting list of plants collected by herself and the late Mr. Mitchell, mostly in the vicinity of Southold, a decade or more ago. Mrs. Frank D. Smith of Peconic, Miss Mabel R. Wiggins of East Marion and William C. Ferguson of Hempstead should be mentioned. The following botanists have visited and collected in the region: Mrs. Agnes Chase, Mr. Wm. T. Davis, Mr. Norman Taylor and others.

\section{INSECT GALLSì}

Asteromyia carbonifera Felt-On leaves of Euthamia tenuifolia.

Caryomyia tubicola O.S.-On leaves of Hicoria glabra at Cutchogue.

Cecidomyia verrucicola O.S.-On leaves of Tilia anericana at Southold.

Dasyneura Lysimachiae Beutm.--On Lysimachia quadrifolia at Orient.

Disholcaspis mamma Walsh-On twigs of Quercus velutina at Greenport.

Eriophyes Cephalanthi Cook-Greenport on leaves of Cephalanthus occidentalis. E. semen Walsh-Orient on leaves of Salix.

Hormomyia canadensis Felt-Cutchogue on leaves of Amelanchier oblongifolia. Lasioptera clavula Beutm.--On twigs of Cornus at Cutchogue, Greenport and Southold.

[No.6, Vol. 20, of TorReYA, comprising pp. I07-I40, was issued 7 February I92I]

* The preliminary flora was published in TorReya 14: 20I-225. Nov. r9i4, and 229-254. Dec. I9I4. The First Supplementary List was published in Torreya I7: III-I22, July I9I7.

$\dagger$ The majority of these galls were named by Dr. E. P. Felt, state entomologist of the State of New York. 
L. nodulosa Beutm,-Orient on stems of Rubus.

Livia maculipennis Fitch-On the inflorescence of Juncus canadensis.

Neolasioptera ramuscula Beutm.-On stems of Doellingeria umbellata at Orient.

Phylloxera Caryaesemen Walsh-Orient on the under surface of the leaves of Hicoria glabra.

Rhabdophaga strobiloides Walsh-On the tips of branches of Salix humilis at Peconic.

Rhopalomyia hirtipes O.S.-On aerial stems of Solidago juncea at Cutchogue and Peconic.

\section{EUPHYCEAE}

Antithamnion americanum (Harv.) Farl.-In the Sound at Orient; determined by Dr. M. A. Howe.

Nitella intermedia Nordst.-Great Pond, Southold; determined by Dr. Howe who has examined specimens twice, and says, "this species or something close to it."

\section{РнyсомyCETES}

Empusa americana Thaxt.-On blow-flies, Calliphora vomitoria at Orient; determined by Prof. John Dearness. Many thousands of these flies are killed by this fungus during cold, wet spells in the summer. They are usually found clustered on the softer parts of grape vines.

Rhysotheca Haldstedii (Farl.) Wils.-On leaves of Helianthus in woods at Orient; determined by Prof. Dearness.

\section{Ascomycetes (Excluding Pyrenomycetes)}

Cudoniella marcida (Müll.) Sacc.-On earth in rich woods at Cutchogue. September. Determined by Dr. C. G. Lloyd as Leotia marcida Pers.: Mycol. Notes 63: 964. May 1920.

Geoglossum Farlowi Cke.-On earth in rich woods at Greenport. October. Determined by Dr. Lloyd who says "it is a very rare plant with spores 3 -septate, 80 mic. long in these." It is difficult to believe, however, that it is other than a spore variation of the more common Geoglossum hirsutum Pers.

Peziza odorata $\mathrm{Pk}$. - On ashes in a cellar at Orient; determined by Dr. Charles E. Fairman.

Phialea scutula (Pers.) Gill._-On dead herbaceous stems at Orient; determined by Dr. Fairman.

Pseudophacidium Betulae Rehm.-On twigs and small branches of Betula populifolia at Orient. Spring. Determined by Dr. Fairman, who says the find is noteworthy; and confirmed by Dr. E. J. Durand, who reports that it agrees with Rehm's Ascomyceten No. 866 in his herbarium.

Tapesia sanguinea (Pers.) Fckl.-On wood of Juniperus virginiana at Orient; determined by Dr. Fairman.

\section{Ascomycetes (Pyrenomycetes)}

Anthostoma gastrinum (Fr.) Sacc-Don dead branches of Amelanchier canadensis at Orient; determined by Prof. Dearness. 
Botryosphaeria fuliginosa (M. \& N.) E. \& E.-Orient on stems of Smilax rotundifolia; determined by Prof. Dearness. No. 2107.

Camarosporium Robiniae (West.) Sacc.-Orient on Robinia Pseudo-acacia, associated with Cucurbitaria elongata (Fr.) Grev. No. 702. N. Y. State Mus. Bull. 197: 25. 1918.

Cryptospora aculeans (Schw.) E. \& E.--On stems and twigs of Rhus copallina and Toxicodendron radicans at Orient; determined by Prof. Dearness.

Diaporthe (Chorostate) cercophora (Ell.) Sacc.-On dead twigs and branches of Celtis occidentalis at Orient; determined by Prof. Dearness.

Diaporthe (Euporthe) cryptica Nitschke-Orient on stems of Lonicera japonica; determined by Prof. Dearness.

Diaporthe (Euporthe) euspina (C. \& E.) Sacc.-Base of stems of Chenopodium ambrosioides at Orient; determined by Prof. Dearness.

Diaporthe (Chorostate) oxyspora (Pk.) Sacc.-On twigs and branches of Ilex verticillata at Orient. N. Y. State Mus. Bull. I97: 38. I9I8. (Diaporthe ocularia (C. \& E.) Sacc.)

Diatrype Baccharidis Earle-Orient on stems and branches of Baccharis halimifolia; determined by Prof. Dearness. No. 4033.

D. disciformis (Hoffm.) Fr.-On branches of Myrica caroliniensis at Orient; determined by Prof. Dearness.

Diatrypella verrucaeformis (Ehrh.) Nitschke-On trunks of Myrica caroliniensis at Orient; determined by Dr. Fairman.

Didymosphaeria Celtidis E. \& E.-On twigs of Celtis occidentalis at Orient; determined by Prof. Dearness.

Dothidea collecta (Schw.) E. \& E.-Orient on twigs of Iva frutescens; determined by Prof. Dearness.

Erysiphe Cichoracearum DC.-On leaves and stems of Plantago Rugelii at Orient; determined by Dr. Fairman.

Eutypa leucostroma (Mont.) Sacc.-On stems of Smilax rotundifolia at Orient; determined by Dr. Fairman.

E. sepulta (B. \& C.) E. \& E.-Orient on stems of Smilax rotundifolia; determined by Prof. Dearness.

Eutypella cerviculata (Fr.) Sacc.-On branches of Celtis occidentalis at Orient; determined by Prof. Dearness.

E. Gleditschiae Berl.-On dead twigs of Gleditschia triacanthos at Orient. No. 724. N. Y. State Mus. Bull. 197: 29. 1918.

E. scoparia (Schw.) E. \& E.-Orient on twigs of Robinia Pseudo-acacia; determined by Prof. Dearness. No. I04I.

E. venusta (Ell.) Sacc-Drient on twigs of Robinia Pseudo-acacia; determined by Prof. Dearness. No. II98.

Gibberella pulicaris (Fr.) Sacc.-On stalks of Zea Mays at Orient; determined by Dr. Fairman.

Gloniella ovata (Cke.) Sacc.-On decorticated and weathered wood of Castanea dentata at Orient. "The type of this species (collected by Ravenel in Carolina) is said to be on oak." No. 824. N. Y. State Mus. Bull. 205-206: 5 I. I9 9.

Gloniopsis Cookeana (Ger.) Sacc.-Orient on dead wood of Quercus alba, 
dead branches of Myrica caroliniensis, dead decorticated branches of Rhus glabra and Xolisma ligustrina. N. Y. State Mus. Bull. 197: 39. 1918.

Guignardia Bidwellii (Ellis) Viala \& Ravaz-On fruit of cultivated grapes; determined by Prof. Dearness and Dr. Fairman. It is associated with Phoma wicola B. \& C. which Rostrup states is a stage of Guignardia Bidzellii.

Hypocrea rufa (Pers.) Fr.-A Corticium-like plant growing on oak; determined by Prof. Dearness. No. 3387 .

Hypoxylon multiforme $\mathrm{Fr}$ ? $-\mathrm{O}$ - wood of Quercus velutina at Orient; determined by Dr. Lloyd (printed): Letter 67:7. July i918.

H. rubiginosum (Pers.) Fr.-Orient on dead branches of Rhus copallina; determined by Prof. Dearness.

Hysterographim Lesquereuxii (Duby) Sacc.-On dead branches of Gleditschia triacanthos at Orient. N. Y. State Mus. Bull. 197: 30. 1918.

H. Vaccinii (Schw.) Fairman-This combination was made in the First Supplementary List in Torreya 17: II3. July 1917: but was wrongly spelled Hysteriographium Vaccinii.

Massaria conspurcata (Wallr.) Sacc.-On twigs of Padus virginiana (Prunus serotina Ehrh.) at Orient. Determined by Dr. Fairman, who says, "spores 60-65 $\times$ i 3.5-14 $\mu$." According to Ellis \& Everhart, your specimen has spores agreeing more with those distributed by Dr. Rehm (in his Ascomyceten) than what Ellis noted in this country. That is, they are about the same width as foreign specimens and wider than those usually found here.

Massarinula Brassicae Dearn. \& House-On dead stems of Brussels Sprouts, Brassica oleracea gemmifera, at Orient. September I9I5. Type in the herbarium of the N. Y. State Museum. Described in N. Y. State Mus. Bull. 197: 31. 19I8.

Microsphaera Alni (Wallr.) Salmon-The var, Vaccinii (Schw.) Salmon on leaves of Vaccinium corymbosum at Orient; determined by Prof. Dearness.

Phyllachora Graminis (Pers:) Fck1.-The var. Panici (Schw.) Spear on leaves of Panicum cladestinum, common throughout the town; determined by Prof. Dearness.

P. Pteridis (Reb.) Fck1.-On fronds of Pteridium aquilinum at Mattituck; determined by Prof. Dearness.

Pyrenophora calvescens (Fr.) Sacc.-On Chenopodium ambrosioides; determined by Prof. Dearness.

Rosellinia protuberans Karst.-Orient on wood of Baccharis halimifolia; determined by Dr. Fairman.

R. pulveracea (Ehrh.) Fckl.-On twigs of Celtis occidentalis; determined by Prof. Dearness.

Sphaerella pardalota C. \& E.-Orient on old leaves of Myrica caroliniensis; determined by Dr. Fairman.

Trematosphaeria nuclearia (DeNot.) Sacc.-On decaying nuts of Hicoria glabra at Orient; determined by Prof. Dearness. No. I202.

Valsa Liquidambaris (Schw.) Cke.-On dead stems of Hamamelis virginiana at Orient. "A new host species. The asci are $30-33 \times 8 \mu$, the spores eight in an ascus, 8-9 $\times 2 \mu$, hyaline, allantoid." N. Y. State Mus. Bull. I97: 45. 1918 . 
V. Pini (A. \& S.) Fr.-On dead bark and twigs of Pinus Strobus at Greenport; determined by Prof. Dearness. The fallen trunk of one tree that had been cut about a year was completely covered with this species, abundantly fruiting.

V. subclypeata C. \& P.-Orient on dead branches of sassafras; determined by Próf. Dearness.

Xylaria corniformis Fr-On buried roots of Quercus velutina at Orient; determined by Dr. Lloyd (printed): Letter 66:4. Oct. I917.

\section{HYPOMYCETES}

Cercospora Acalyphae Pk.--Orient on leaves of Acalypha gracilens; dètermined by Prof. Dearness.

C. circumscissa Sace:-Common at Orient on leaves of Padus virginiana (Prunus serotina); determined by Prof. Dearness.

C. copallina Cke:-Cutchogue on leaves of Rhus copallina; detérmined by Prof. Dearness who says, "this is likely only a synonym of Cercospora rhoina C. \& E."

C. rhoina C. \& E.--On leaves of Rhus copallina at Cutchogue; determined by Prof: Dearness.

Cladosporium herbarum (Pers.) Link-Orient on leaves of Hemerocallis fulva; determined by Prof. Dearness.

Exosporium Tiliae Link-Orient on dead branches and trunks of Tilia vulgaris; determined by Prof. Dearness.

Fusarium Celtidis Ell. \& Tracy-Orient on twigs of Celtis occidentalis; determined by Prof. Dearness.

\section{Melanconiales}

Cylindrosporium Iridis $\mathrm{E} . \& \mathrm{H}$-On living leaves of Iris versicolor at Orient. N. Y. State Mus. Bull. $197: 27$. 1918.

Gloeosporium Opuntiae E. \& E.-On leaves of Opuntia; determined by Dr. Fairman. Large patches of the Eastern Prickly Pear have been killed by this fungus at Orient.

Marsonia Potentillae (Desm.) Fiseh.-Greenport on leaves of Potentilla canadensis; determined by Dr. House.

Melanconium betulinum Schm. \& Kze.-On twigs of Betuld populifolia at Greenport; determined by Dr. Fairman.

Pestalozzia uncinata E11. \& Kell.-On leaves of Quercus velutina; determined by Dr. House.

Stagonospora Chenopodii $\mathrm{Pk}$ - (Phleospora Chenopodii $\mathrm{E}$. \& $\mathrm{K}$.) On leaves of Atriplex hastata, common at Orient; determined by Prof. Dèarness.

Steganosporium acerinum Pk.-Orient on dead branches of Acer PseudoPlatanus. Determined by Prof. Dearnéss who says, "may be a synonym of Steganosporium piriforme (Hoffm.) Cda.: Mr. Ellis used to eall the larger spore form $S$. cellulosum Cda. and the smaller spore form $S$. piriforme. The spores are $36 \times$ i $8 \mu_{\text {." }}$ 


\section{SPHAEROPSIDEAE}

Coniothyrium concentricum (Desm.) Sacc.-On leaves of cultivated Yucca at Orient; determined by Prof. Dearness.

Leptostromella Chenopodii Dearn. \& House-Orient on dead stems of Chenopodium album. Described in N. Y. State Mus. Bull. 205-206: 53-54. I9I9.

Macrophoma celtidicola Dearn. \& House-Orient on twigs of Celtis occidentalis; determined by Prof. Dearness.

Phlyctaena arcuata Berk.-Orient on dead stems of Helianthus annums. No. 726. "Spores filiform arcuate to falcate, $25 \mu$ long." N. Y. State Mus. Bull. 205-206: 55. I9I9.

Phoma Celtidis Cke.-On twigs of Celtis occidentalis; determined by Prof. Dearness. No. 3388 .

Phyllosticta Chenopodii Sacc.-On leaves of Chenopodium album at Orient; determined by Dr. Fairman.

P. Kalmicola Schw.-Greenport on leaves of Kalmia latifolia; determined by Prof. Dearness.

Phyllosticta limitata $\mathrm{Pk}$.-On leaves of apple, Malus; determined by Prof. Dearness. Very abundant throughout the town during the summer of I9I9: and practically defoliating some trees.

P. minima (B. \& C.) E. \& E.-Laurel on leaves of Acer rubrum; determined by Dr. Fairman.

P. Sassafras Cke.-On leaves of Sassafras, common throughout the township. Determined by Prof. Dearness who says, "Ellis and Everhart in their North American Phyllostictas say the specimens available for examination are all sterile and that the species must be put in the doubtful class. The spots on some of these leaves have pycnidia with the small spores of the description; but most of the spots are sterile."

Septoria brunneola (Fr.) Niessl.-Cutchogue on leaves of Vagnera racemosa; determined by Prof. Dearness.

S. Macrosporia Dearn.-On leaves of Chrysanthemum Leucanthemum at Orient. Prof. Dearness says, "externally it is exactly like it but the spores fall short in average size."

S. mollisia Dearn \& House-Mattituck on leaves of Antennaria plantaginifolia; determined by Prof. Dearness, who says this may be the same as Fairman's Septoria lanaria.

S. Polygonorum Desm.-Orient on leaves of Persicaria Persicaria; determined by Prof. Dearness.

S. Stellariae Rob. \& Desm.-On leaves of Alsine media at Orient. Determined by Prof. Dearriess who says, "the same as Fungi Columb. No. 775, which Mr. Ellis named as the above. It is not very distinct from Septoria Silenicola Ell. \& Mart."

S. Violae West.-Greenport on leaves of Viola cucullata; determined by Prof. Dearness.

Sphaeronema Robiniae B. \& C.-On twigs and branches of Tilia americana at Orient; determined by Prof. Dearness. 
Sphaeropsis Celtidis E. \& E.-On twigs of Celtis occidentalis at Orient. No. 3561. Determined by Dr. Fairman who says, "Cfr. Am. Nat. 428, I897 and Saccardo Syl. 14: 921. I have never had this before: it was originally named from a specimen collected by Bartholomew on Celtis occidentalis in Kansas."

S. Syringae C. \& E.-Orient on twigs of Syringa vulgaris; determined by Dr. Fairman.

Vermicularia herbarum West.--On old stems of Geranium maculatum at Orient; determined by Prof. Dearness.

\section{USTILIGINACEAE}

Sorosporium Syntherismae (Pk.) Farl.-Orient on Panicum dichotomiflorum; determined by Dr. G. P. Clinton.

\section{UREDINACEAE*}

Peridermium Peckii Thüm.-Common. On Azalea viscosa at Greenport, Peconic and Southold. On Gaylussacia baccata at Cutchogue. (Pucciniastrum Myrtilli (Schum.) Arth.)

$P$. pyriforme $\mathrm{Pk}$.-Found sparingly during August on leaves of Comandra umbellata at Mattituck. (Cronartium Comandrae $\mathrm{Pk}$.)

Pucciniastrum Agrimoniae (Schw.) Tranz.-Orient on leaves of Agrimonia gryposepala.

P. Pyrolae (Pers.) Diet.-Southold on Chimaphila maculata; but one collection found.

\section{Puccintaceae}

Puccinia Anemones-Virginianae Schw.-On leaves of Anemone Virginiana at Indian Neck, Peconic. August.

P. Circaeae Pers.-Orient on leaves of Circaea Lutetiana.

P. Ellisiana Thüm.-Orient on Schizachyrium scoparium. November.

$P$. investita Schw.-On Gnaphalium obtusifolium at Cutchogue, Orient and Peconic. August.

P. minutissima Arth.-Mattituck on stems and leaves of Decodon verticillatus. August. Very common in one swamp and forming large swellings on stems and the midveins of leaves. (Aecidium Nesaeae Ger.)

P. patruelis Arth.-On leaves of Lactuca canadensis at Orient. June. Dr. Arthur says, "lately has been called Puccinia hieraciata (Schw.) Jackson. This is rather a rare rust in New York and in fact throughout the Atlantic states: but is very common in the interior. It has telia on various species of Carex.".

Uromyces Hyperici-frondosi (Schw.) Arth.-Gardiner's Island on leaves of Hypericum mutilum; determined by Burnham.

$U$. Lespedezae-procumbentis (Schw.) Curt.-On Lespedeza capitata at Cutchogue, Peconic and Southold. On Lespedeza virginica at Cutchogue. Locally common at these stations. (Nigredo Lespedezae-procumbentis (Schw.) Arth.)

* Unless otherwise stated the Rusts were determined by Dr. J. C. Arthur. 
U. Polemonii (Pk.) Barth.-N. Y. State Mus. Bull. 197: 13. 1918, as a contribution; probably on Spartina stricta alterniflora.

\section{TREMELLACEAE}

Dacryomyces deliquescens (Bull.) Duby-On old wood of Juniperus virginiana; determined by Dr. Lloyd: Mycol. Notes 63: 964. May 1920.

Exidia recisa Fr. On branches of Quercus velutina at Orient; determined by Dr. Lloyd: Mycol. Notes 63: 964. May 1920.

Naematelia nucleata (Schw.) Fr.-On old bark of Quercus velutina; determined by Dr. Lloyd (printed): Letter 66:4. Oct. I9I7.

\section{THELEPHORACEAE}

Aleurodiscus nivosus (B. \& C.) v. Höhn \& Litsch.-On bark of Juniperus vir giniana at Orient. No. I89. (In Mo. Bot. Gard. Herb., 44228) (Stereum acerinum Pers., var. nivosum B. \& C.) Ann. Mo. Bot. Gard. 5: 195. I918.

Craterellus cornucopioides (L.) Pers.- "Note 862. The common Craterellus cornucopioides is usually so regular and cup shaped that we were somewhat surprised to receive a collection lobed and almost divided at the base, from Mr. Latham. We supposed that it had been torn accidentally but Mr. Latham stated that it grew naturally in this way and he found a large colony of this form." Dr. Lloyd's Mycol. Notes 63: 965. May 1920.

Cyphella muscigena (Pers.) Fr.-Thuidium paludosum has been found "only in one locality, a blackish meadow in Orient. It is common there, but rarely fruiting. It is a frequent host of Cyphella in this plot. There are several other species of musci associated with the Thuidium. It is interesting that the fungus should go commonly to this single species and not at all to the others." Bryol. 23: 7. Jan. 1920. Determined by Dr. Fairman.

Hymenochaete agglutinans Ellis-On Sassafras; determined by Prof. Dearness. H. corrugata (Fr.) Lev.-Orient. No. I54. (In Mo. Bot. Gard. Herb., 44229.) Determined by Dr. E. A. Burt. Ann. Mo. Bot. Gard. 5: 36I. I9I8.

Thelephora multipartita Schw.--On earth in woods at Orient; determined by Dr. Lloyd: Mycol. Notes 63: 965. May 1920.

Thelephora spiculosa Fr.-On earth in dry woods at Cutchogue; determined by Dr. Lloyd who says " rare."

Tremellodendron merismatoides (Schw.) Burt-On heavy soil in woods at Orient; determined by Dr. Lloyd.

\section{HydNACEAE}

Hydnum caryophylleum B. \& C.-On old wood of Hicoria glabra at Orient; determined by Prof. Dearness.

H. vellereum Pk.-In dry woods at Cutchogue. Dr. Lloyd says, "quite fragrant when received": Mycol. Notes 63: 964. May 1920, as Hydnum amicum Quel.

H. zonatum of American Mycology-In dry woods on earth at Cutchogue. Determined by Dr. Lloyd: Mycol. Notes 63: 964. May 1920, as Hydnum scrobiculatum Fr. 
Phlebia merismoides Fr.-Orient on Prunus Avium; determined by Dr. Lloyd (printed): Letter 69:7. April I9I9.

Radulum pallidum B. \& C.-On underside of a decayed log of Pinus Strobus in a swamp at Greenport; determined by Dr. Llayd (printed): Letter 69: 7. April I919.

\section{POLy PORACEAE}

Daedalea ochracea Lloyd-On oaks at Cutchogue; determined by Dr. Lloyd: Mycol. Notes 63: 964. May 1920. Under Note No. 137, Dr. Lloyd says, "I would designate the light colored forms of Daedalea unicolor... which correspond to Polystictus ochraceus as forms of Polystictus hirsutus."

Merulius bellus B. \& C.-Orient, "comm. by N. Y. State Herb., P66 (in Mo. Bot. Gard. Herb., 43604)." Ann. Mo. Bot. Gard. 4: 332. Nov. I9I7.

Merulius brassicaefolius Schw.-On earth in a cellar at Orient; determined by Dr. Lloyd.

Polyporus brumalis (Pers.) Fr.-Greenport on Vaccinium; Orient on wild cherry; and Southold on Sambucus canadensis.

P. (Ganoderma) Curtisii Berk.-On trunks of living apple tree. Determined by Dr. Lloyd who șays, "this is a southern unvarnished form of Polyporus lucidus, it is quite common in the south, but rarely found as far north as with you" (printed): Letter 67: 7. July I9I8. Previously reported as Ganoderma psendoboletus (Jacq.) Murrill.

P. pocula (Schw.) B. \& C.-Orient on living bark of Quercus velutina at Orient. Found growing in clusters in April. Determined by Dr. Lloyd who says, “a unique little species": Mycol. Notes 63. 965. May 1920.

P. stipticus (Pers.) Fr.-On wood of Quercus velutina at Orient; determined by Dr. Lloyd (printed): Letter 66:4. Oct. 1917.

$P$. trabeus Rostk.-On wood of Quercus velutina at Orient; determined by Dr. Lloyd (printed) : Letter 67: 7. July I918.

Polystictus dependens B. \& C.-On the underside of a $\log$ of Pinus rigida at Cutchogue. September. No. 2080. A colony of about a dozen plants ranging from $I / 4$ to $3 / 4$ of an inch in diameter. "Note 86r. A rare species (Cfr. Stip. Polyporoids, p. I65) only known from a few stations in the south. This is the only collection in any way northern. Otherwise it is only known from one collection from Japan": Dr. Lloyd's Mycol, Notes 63: 965. May i920.

Polystictus hirsutus (Wulf.) Fr,-The form Polystictus hirsutulus Schw, on Quercus velutina at Orient; determined by Dr. Lloyd (printed): Letter 69: 7. April i919.

Poria omoema Berk-On limbs of Betula populifolia at Orient; determined by Prof. Dearness who says a similar plant was named this species for him by Mr. Ellis. The type of this species was collected on pine in South Carolina by Mr. Ravenel. (=Poria subacida (Pk.) Sacc.)

\section{AGARICACEAE}

Crepidotus applanatus (Pers.) Fr.-Orient on trunks of Quercus velutina; determined by Dr. Lloyd (printed): Letter 67:7. July I9I8. 
Lenzites albida $\mathrm{Fr}$-On trunk of Acer rubrum at Greenport; determined by Dr. Lloyd who says, "the old, bleached white, wintered, lenzitoid form of Daedalea confragosa."

L. corrugata Klotsch.-Orient on oaks and Sassafras; determined by Dr. Lloyd (printed): Letter 69: 7. April 1919.

Panus strigosus B. \& C.-Formerly reported from Orient as Panus levis Berk. The Orient plant is figured in Dr. Lloyd's Mycol. Notes 52: 746. fig. I1 20. Dec. I917.

Pleurotus niger Schw.-On terminal branches of Rhus copallina at Orient. Plants $1 / 8$ of an inch in diameter and slaty black. Dr. Lloyd says it is rare (printed): Letter 69: 7. April I9I9.

$P$. sapidus Klachb.-On stumps of Hicoria glabra at Orient; determined by Dr. Lloyd (printed): Letter 69: 7. April 1919, and Mycol. Notes 63: 965. May 1920.

\section{GASTEROMYCETES}

Calvatia lilicina Berk.-On earth in rich woods at Orient; determined by Dr. Lloyd: Mycol. Notes 63: 965. May 1920.

Dictyophora duplicata (Bosc) Ed. Fisch.-On earth in moist woods at Cutchogue, Orient and Southold; determined by Dr. Lloyd as Phallus duplicatus: Mycol. Notes 63: 964. May 1920.

Lycoperdon gemmatum Batsch-On pure sand at Orient; determined by Dr. Lloyd.

Scleroderma Cepa Pers.-On pure sand in shade of pines and open ground at Southold; determined by Dr. Lloyd: Mycol. Notes 63: 964. May 1920.

\section{Musci}

Amblystegium varium (Hedw.) Lindb.-Orient at the base of a hickory tree about a moist cavity; determined by Mr. G. B. Kaiser.

Fontinalis gigantea Sulliv.-Swamp woods in water at Mattituck; determined by Dr. A. J. Grout. No. 1736.

Mnium cinclidioides Hüben.-In a swamp at Mattituck. No. I843. Determined by Dr. Grout who says, "a depauperate form .... the first to be reported from Long Island so far as I know, although it apparently is frequent along the west bank of the Hudson river."

\section{PolyPodiaceae}

Adiantum pedatum L.-Southold, localized in moist woods south of Great Pond. The reference to this species in the first part of this Flora was an error: the above record is the only known station in the town. It was first discovered many years ago by Miss Mary H. Huntting and reported by Mrs. Frank D. Smith.

Polypodium vulgare L.-Sandy soil at Orient. No. 2331.

Polystichum acrostichoides (Mx.) Schott-Rare in woods south of Great Pond, Southold, Sept. Io, 1919. No. 4088. 


\section{LYCOPODIACEAE}

Lycopodium adpressum (Chapm.) Lloyd \& Underw.-Southold in a sandy bog. No. 3455 .

L. obscurum L.-Moist woods at Orient and Southold.

(To be continued) 

[Reprinted from Torreya, Vol. 2i, No. 2, March-April, I92i.]

\title{
THE FLORA OF THE TOWN OF SOUTHOLD, LONG ISLAND AND GARDINER'S IELAND
}

\author{
By Steward H. Burinham and Roy A. Latham \\ (Continued from January-February TorReYA)
}

SPERMATOPHYTA

Picea rubens Sarg.-On Gid's Island, July 24, 1920 (Dr. C. S. Gager, N. Taylor \& R. Latham). This island does not cover over three acres and is entirely surrounded by salt marshes. Two of the four trees are dead and the other two more than half dead: but there are four little seedlings ten to twenty inches high. Mr. Taylor remarks that these are evidently the last remains of what was once a spruce forest covering the whole island and that they are putting up a losing fight.

Pinus Strobus L.-A colony of nearly 300 trees in a swamp at Greenport; some of the trees actually growing where their roots are submerged a portion of the year. November 19I8. Mr. Price, an elderly gentleman, who owns the swamp, says his father told him that they. were a true native here. Some of the trees are probably Ioo years old. There are eleven trees in dry woods at Southold which may be native. During August I920 several hundred trees were seen in dry wood-lands at Bay View.

Sparganium androcladon (Engelm.) Morong-Wet place, Gardiner's Island. No. 3433. Sept. 20, 1920.

Potamogeton diversifolius Raf.-In a pond on Gardiner's Island. No. 3427. *Agrostis altissima (Walt.) Tuck.—Low marshy ground, rare at Mattituck.

A. perennans (Walt.) Tuck.-Dry soil throughout the town.

Aristida tuberculosa Nutt.-Rare along the railroad track in ashes at Laurel in the western part of the town. It is abundant in sandy soil a few miles further west but outside the town of Southold.

Calamagrostis cinnoides (Muh1.) Scribn.-Not common in low open ground at Mattituck.

Festuca Myuros L.-Wet sandy soil at Mattituck.

F. rubra L.-Orient in rather dry open woods near a salt marsh.

Miscanthus sinensis Anderss.-Occasionally found in waste places and old yards.

Panicularia obtusa (Muh1.) Ktze--Mattituck in a swamp.

Panicum meridionale Ashe-In dry woods at Cutchogue; determined as Panicum albemarlense Ashe.

P. tennesseense Ashe-Southold in sandy soil.

* The grasses were named by Mrs. Agnes Chase of the U. S. Department of Agriculture. 
Carex atlantica Bailey-Wet woods at Greenport and Orient; determined by Mr. G. P. Van Eseltine. No. 2285.

C. festucacea Schkr-Wet open place in woods at Greenport (No. 3518) and at Southold (No. 3530).

Carex laevivaginata (Küken.) Mackenzie-Greenport in wet woods; determined by Mr. Van Eseltine. No. 2288.

C. lanuginosa Mx.-Greenport in a swamp and Orient in wet sandy woods; determined by Mr. Van Eseltine. No. 2339.

C. laxiculmis Schwein.-Frequent in dry open woods at Southold. No. 3524.

C. Swanii (Fernald) Mackenzie-Orient. No. I I0I.

Cyperus dentatus Torr.-Wet sandy shores at Laurel. No. 1278 and 3447 .

Eleocharis acicularis (L.) R. \& S.-Salt marsh, Gardiner's Island (No. 3432) and sandy shore of a pond at Laurel (No. 3469).

Eriophorum virginicum L.-Laurel. No. 1287. Aug. 4, 1918.

Rynchospora alba (L.) Vahl-Laurel. No. I29I.

Spirodela polyrhiza (L.) Schleid.-East Marion (No. 3533) and Greenport. Locally common on woodland pools and ponds: at Greenport abundantly associated with Lemna minor L.

Juncus aristulatus $\mathrm{Mx}$, - Growing in large clumps in a brackish marsh at Orient. Rare. May 30. 1917. No. 1087.

J. tenuis Willd.-Dry hills, Gardiner's Island. July I4, I918. No. I260.

Aletris farinosa L.-Laurel. Sept. I0, 1917. No. I077.

Lilium philadelphicum L.-Open ground between Southold and Great Pond at Peconic. A colony of about 50 plants.

Gymnadeniopsis clavellata (Mx.) Rydb.-Boggy woods at Mattituck. Aug. 28, I920. No. 3460 .

Ibidium gracile (Bigel.) House--Plants having a single stout root were found in dry open ground at Southold. Aug. 20, I9zo. No. 3434.

Hicoria ovata (Mill.) Britton-Low woods, Gardiner's Island. No. 3422. Sept. 20, I920.

Myrica Gale L--Laurel. Aug. 4, 1918. No. I288.

Populus heterophylla L.-Greenport in swampy woods. July 25, 1920. The first time Mr. Taylor has seen it wild on Long Island.

Salix Bebbiana Sarg.-Greenport in dry open places. Plants commonly two feet high or less were found on sandy dunes at Southold.

S. cordata Muhl.-In a swamp at Mattituck. June 18, 1920. No. 3521. The leaves little narrower than usual.

S. discolor Muhl.-The var, eriocephala ( $\mathrm{Mx}$.) Anders. In open places at Greenport (Wm. C. Ferguson); determined by Dr. P. A. Rydberg.

Quercus ilicifolia Wang.-Rare near Laurel in light soil. No. 1268. Aug. 4, I9I8. A single plant at Cutchogue; which is the easternmost record for it. The scrub oak becomes abundant about eight miles west of the Southold town limits.

Q. prinoides Willd.-Southold. Sept. Io, I9I9. Rare in dry woods at Mattituck and Peconic.

Ulmus fulva Mx.-Wet woods at Greenport. July 20, 1920. No. 3459. Very rare in low woods; several trees in a bunch, which came from the stump of a large tree, cut many years ago. 
Morus rubra L.-Was listed previously as probably introduced; but now found to be a native on Gardiner's Island.

Boehmeria Drummondiana Weddell-Laurel. Sept. I0, I917. No. 1280.

Persicaria opelousiana (Riddell) Small-Swampy woods at Greenport. Aug. 28, 1920. No. 3386.

$P$. orientalis (L.) Spach.-Occasional in waste grounds and cultivated fields at Greenport and Orient.

Polygonum atlanticum (Robins.) Bickn.-Large bushy plants on sea beaches at Orient. Aug. 20, I920. No. 3396.

Rumex mexicanus Meisn.-Orient. June I9r6. Specimens previously reported as Rumex pallidus Bigel, should probably be referred here.

Acnida cannabina L.-Salt marshes at Laurel. Sept. I4, I9I8. No. I3I3. Specimens previously reported as Acnida tuberculata Moq. should probably be referred here.

Chenopodium Botrys L.-Southold (Mrs. F. R. Mitchell); determined by Mr. Taylor.

C. rubrum L.-Beach at Southold (Mrs. Mitchell); determined by Mr. Taylor. Allionia nyctaginea Mx.-The subspecies Allionia nyctaginea ovata (Pursh) Morong. Moist waste ground at Laurel. Sept. I4, 1918. No. I318.

Cerastium arvense L.-Fields at Southold (Mrs. Mitchell).

Silene stellata (L.) Ait.-Moist woods at Southold. Oct. I9, I9I9. No. 407ז.

Magnolia tripetala L.-A single tree twenty feet high with trunk diameter of five inches, at the edge of wet woods at Southold. The origin is uncertain, but introduced. Reported to Mr. Latham by Mrs. Mitchell. Oct. r6, 1919. No. 2148 .

Cardamine hirsuta L.-Old lawn at Southold (Mrs. Mitchell).

Draba caroliniana Walt.-Sandy soil at western end of Long Beach at Orient. Rare. 23 May-early June, i92o.

Sarracenia purpurea L,-A single plant from a bog near Mattituck.

Agrimonia Bicknellii (Kearney) Rydb.-Rare in dry woods at Southold (Ferguson \& Latham); determined by Dr. Rydberg.

A. rostellata Wallr.-Uncommon in dry woodlands at Southold. Oct. ro, r9r9. No. 2120.

Potentilla recta L.-Dry roadsides at East Marion. Rare. June 20, I920. No. 353 I.

Amelanchier oblongifolia (T. \& G.) Roem.-Not uncommon in dry woods at Cutchogue; determined by Dr. K. M. Wiegand. No. 3407.

Crataegus Arnoldiana Sarg.-Gardiner's Island, frequent at margins of woods in dry or wet soil; determined by Mr. W. W. Eggleston. A thick foliaged, beautiful, round-topped tree about twenty feet high.

Crataegus intricata Lange-Gardiner's Island; determined by Mr. W. W. Eggleston.

Crataegus intricata Lange.-Gardiner's Island; determined by $\mathrm{Mr}$. W. W Eggleston.

Chamaecrista nictitans (L.) Moench.-Bay View and Southold, locally common. Sept. 1920. No. 3403.

Crotalaria sagittalis L.-Common on a dry sandy ridge at Southold. Aug. 28, 1920. No. 3385 . 
Lathyrus latifolits L. - A rare escape in dry woods in the vicinity of an old house-site at Cutchogue. Aug. 21, I920. No. 3399.

Meibomia obtusa (Muhl.) Vahl-Dry hillside, locally common at Southold. Aug. 29, 1920. No. 3395 .

M. rigida (L.) Ktze.-Mattituck. Aug. 9, I918. No. 1284 .

Polygala Nuttallii T. \& G.--Mattituck. Aug. 9, 1918. No. 1267.

Tithymelus Helioscopia (L.) Hill-Rare in a field at Peconic (Mrs. Smith). Dec. 10, 1920.

T. Ipecacuanhae (L.) Small-Sandy soil at Laurel. Aug. 4, 1918.

Staphylea trifolia L.-Rocky woods, Southold; determined by Mr. Taylor. Aug. I, 1920. No. 3548.

Hudsonia ericoides L.-Common in one locality at Bay View. Aug. 21, I920. No. 3400 .

Rotala ramosior (L.) Koehne-A small colony in wet sand north of Great Pond, Southold. Oct. 19, 1919. No. 2126.

Myriophyllum humile (Raf.) Morong-Gardiner's Island in a pond. Sept. 20, 1920. No. 3426 .

Cicuta bulbifera L.-Mattituck. Aüg. 9, 1918. No. 1270.

Cormus Amomum Mill.-Uncommon in rich woods at East Marion (Miss Mabel R. Wiggins); verified by Mr. Taylor.

Chamaedaphne calyculata (L.) Moench-Laurel. Aug. 4, I9i8. No. I269.

Eubotrys racemosa (L.) Nutt.-Wet woods at Southold. Oct. 19, 1919. No. 4068 .

Gaulthera procumbens L.-Mattituck.

Neopieris mariana (L.) Britton-Low place in woods at Southold. Sept. I4, 1919. No. 2263. Cutchogue in rich woods. Rare on Fleets Neck, Cutchogue but frequent on Nassau Point.

Gaylussacia frondosa (L.) T. \& G.-Dry woods at Mattituck. Aug. 2I, I920. No. 3405 .

Asclepias exaltata (L.) Muhl.-Rare in wet woods at Southold, south of Great Pond. July 30 , I9zo. No. 3565 .

Phlox subulata L.-Escaped in old yards at Orient. Sept. 10, 1920. No. 3428 .

Lithospermum arvense L.-Field at Southold (Mrs. Mitchell); determined at U. S. Dept. of Agriculture. Dry cultivated field at Bay View. May r, I9I9. No. 2158.

Onosmodium virginianum (L.) DC.-Dry or moist woods at Fleets Neck at Cutchogue. Sept. 14, 1919. No. 2246.

Cunila origanoides (L.) Britton-Very rare in oak woods on Fleets Neck at Cutchogue. Sept. I4, 1919. No. 2262.

Koellia flexuosa (Walt.) MacM.-Dry open woods on Gardiner's Island. Sept. 20, 1920. No. 3424 .

K. incana (L.) Ktze.-Common in dry hilly woods at Southold. Oct. 19, I919. No. I909.

K. mutica (Mx.) Britton-Dry woods at Southold. Sept. 2I, 1919. No. 4052. Leonurus Cardiaca L.-Waste places at Bay View. Aug. 21, 1920. No. 34I3. Lycopus rubellus Moench-Greenport and Southold in low woods, frequent. Mentha piperita L.-Roadside at Cuchogue. Oct. I4, 1919. No. 4045. 
Stachys hyssopifolia Mx.-Gardiner's Island. July I4, I918. No. I258.

Physalis heterophylla Nees-Locally common as a weed in light cultivated soil at Cutchogue. Oct. 4, 1919. No. 4047.

Pentstemon digitalis (Sweet) Nutt.-Rare in dry ground at Southold. Aug. 28, 1920. No. 3384 .

Utricularia macrorhiza LeConte-Laurel. Sept. Io, I917. (Utricularia vulgaris of Am. Auth.)

Galium Mollugo L.-Orient. July I5, 1918. No. 125.

G. verum L.-Field at Southold (Mrs. Mitchell).

Viburnum cassinoides L.-Laure1. Sept. I4, I918. No. I316. Rich woods at Greenport. Oct. 30, I920. No. 345I.

Cucurbita Pepo L.-Several specimens growing wild on sand dunes at Southold. Not an uncommon escape on farms and in waste places.

Micrampelis lobata (Mx.) Greene-Orient. Sept. r, I9r8. No. I310.

Sicyos angulatus L.-Rare in waste places at Greenport.

Hieracium aurantiacum L.-Rich soil along old road in woods at Southold. Sept. 29, 1920. No. 3465.

H. marianum Willd.-Dry soil at Southold (Mrs. Mitchell).

Lactuca canadensis L.-The var. integrifolia (Bigel.) Gray in dry woods at Cutchogue (No. 3398), Aug. I5, 1920; and Orient (No. I335), Sept. 30, I9I8. This includes the previously reported $L$. canadensis v. montana Britton and Lactuca sagittifolia Ell.

Lactuca Scariola L.-Dry woods at Bay View. Sept. 5, 1920. No.3436. Also the var. integrata Gren. \& Godr, in sandy places at Orient. Sept. I5, 1920. No. 3418.

Nabalus trifoliolatus Cass., var. obovatus var. nov. Leaves purplish, membranous, obovate or oblanceolate, or deltoid, acute, on margined petioles, slightly denticulate or entire. Moist woods at Orient. Oct. 1919. No. 3375. Plants with deltoid leaves were found in dry ground at Bay View. Aug. 29, I920. No. 3446 .

Aster laevis L.-The var. amplifolius Porter is rare along wet margins of woods at Orient. Oct. xI, I9I9. No. 220r.

A. Lowrieanus Porter-Uncommon in rich woodlands at Greenport and Orient (Mr. Ferguson); verified at the N. Y. Bot. Garden.

A. vimineus Lam,-Dry or wet soil in fields at Gardiner's Island. Sept. I9, 1920. No. 3421.

Centaurea maculosa Lam.-Dry fields at Cutchogue. Oct. 19, I9I9. No.2III.

C. nigra L.-The var. radiata DC. in dry pastures at Cutchogue. Oct. I9, I9I9. No. 2110.

C. solstitialis L.-Field at Southold (Mrs. Mitchell); determined at U. S. Dept. of Agriculture.

Cirsium muticum Mx.-Gardiner's Island in wet woods. Sept. 20, 1920. No. 3429.

Lacinaria scariosa (L.) Hill,-A single plant in dry woods at Fleets Neck, Cutchogue. Sept. 14, 1919. No. 2245.

Solidago ulmifolia Muh1.-Dry hillside at Southold. Sept. I, r920. No. 3394. 

- 

Reprinted from TonkE A, Vol. 23, Nos. 1 \& 2, January-February, March-Aprll, 1923.

THE FLORA OF THE TOWN OF SOUTHOLD, LONG ISLAND, AND GARDINER'S ISLAND, NEW YORK

Stewart H. Burnham and Roy A. Latham

Third Supplementary List*

INSECT GALLS

Amphibolips acuninata Ashm.-Very abundant on Quercus ilicifolia at Laurel; determined by Dr. E. P. Felt.

Cecidomyia viticola O.S.-On leaves of Vitis at Mattituck; determined by Dr. Felt.

${ }^{*}$ The Preliminary flora was published in Torreya 14: 201-225. Nov. 1914 and 229-254. Dec. 1914. The First Supplementary List was published in Torreya 17: III-I22. July 1917. The Second Supplementary List was pub. lished in Torreya 21: I-II. Jan.-Feb. I92I and 28-33. March-April I92I. 
Phytophaga rigidae O.S.-On leaves of Salix discolor at Southold; determined by Dr. Felt.

\section{MYXOMYCETES}

Enteridium splendens Morg.-On wood of Quercus at Orient; determined by Prof. John Dearness.

Hemitrichia stipitata (Mass.) Macbr.-Orient on rotten wood of Quercus velutina; determined by Prof. Dearness.

Physarum cinereum (Batsch) Pers.-On old corn stalks, Zea Mays, at Orient; determined by Prof. Dearness.

\section{EUPHYCEAE}

Chara formosa C. B. Robinson-Great Pond, Southold, on pure sandy bottom in 2 feet of water. No. II8I. Determined by Dr. M. A. Howe who says, "a nearly related species has sometimes been identified as Chara sejuncta $\mathrm{A}$. Br."

Gloiotrichia natans (Hedw.) Rabenh.-Attached to water plants in ponds; determined by Dr. Howe.

Licmophora gracilis (Ehrenb.) ('run.--Rocks at ebb tide, Gardiner's Bay; determined by Chas. S. Boyer.

Microspora stagnorum (Kütz.) Lagerh.-Shallow pool in woods at Greenport; determined by Dr. T. E. Hazen.

Microspora Iumidula Hazen-Shallow pool in woods at Greenport; determined by Dr. Hazen.

Nitella flexilis Ag.- Shallow ponds at Southold; determined by Dr. Howe.

Nitella transilis Allen-Great Pond, Southold, on pure sandy bottom in water 2 feet deep. No. II 80 . Determined by Dr. Howe who says, "a nearly related species has sometimes been identified as Nitella tenuissima (Desv.) Coss. \& Germ."

Synedra tabulata (Ag.) Kü̈z._-Rocks at ebb tide, Gardiner's Bay; determined by Mr. Boyer.

Tribonema bombycinum (Derb. \& Sol.) Hazen-Shallow woodland pool at Laurel; determined by Dr. Hazen.

\section{PHYCOMYCETES}

Phytophthora infestans (Mont.) DeBary-Common on potato, Solanum tuberosum; determined by Prof. Dearness.

\section{ASCOMYCETES (excluding PYRENOMYCETES)}

Aleuria aurantia (Pers.) Fckl.-Bare earth on hills at Orient; determined by Prof. Dearness.

Bulgaria rufa Schw.-On buried wood, Cutchogue; determined by Dr. C. G. Lloyd, who says, "recent writers have proposed to separate this from Bulgaria inquinans on account of its hyaline spores." Reported in Mycol. Notes 65: 1077. Nov. 1920.

Cudoniella marcida (Müll.) Sacc.-On earth in rich woods, Greenport; determined by Dr. Lloyd, who says, "The four species of Leotia we have are distinguished chiefly by the color (compare Geo- 
glossaceae, p. I5). All usually have greenish color or cast at least, but this species impressed us at once by the absence of any green tint. The stipe is white and the head pale brownish, while Leotia marcida usually has a 'greenish olive' head and a yellowish stem. It is the only one of the forms that is not decidedly green, hence we so refer this species rather than to base a new name. The spores are hyaline $(6 \times 20 \mu)$ with no greenish cast. . . When soaked the plant is a very bright color. It develops a faint greenish tint on the stem but none on the head."

Exoascus alnitorquus (Tul.) Sadeb.-@n fruit of Alnus incana at Southold; determined by Dr. H. D. House.

Geoglossum hirsutum Pers.-In wet woods on earth, Cutchogue; determined by Dr. Lloyd; Mycol. Notes 65: 1077. Nov. 1920.

Helotium epiphyllum (Pers.) Fr.-Old leaves in woods at Cutchogue; determined by Prof. Dearness.

Phacidium brunneolum Pk.-On leaves of Galium Claytoni, Gardiner's Island; determined by Prof. Dearness.

Pitya cupressi (Batsch) Fckl.-Orient on Juniperus virginiana; determined by Prof. Dearness. (Lachnella cupresii (Batsch) Phillips.)

\section{ASCOMYCETES (PYRENOMYCETES)}

Anthostomella endoxyloides Fairman-" "On a dead tree of some species of Populus, Orient, N. Y., Sept., 1919, Roy Latham, no. 2073." A new species, described by Dr. Chas. E. Fairman in Proc. Rochester Acad. Sci. 6: 125. April I922.

Diaporthe Peckii Sacc.-Orient on Rhus radicans. No. 3503. Determined by Prof. Dearness, who says, "I do not know of any other collection of this than the one Peck made in I885 at Saugerties, N. Y. Dr. Peck calls this Diaporthe sparsa but Saccardo changed it to Peckii."

Eutypella angulosa Nitsch-Orient and Greenport on trunks and branches of Betula populifolia; determined by Prof. Dearness.

Eutypella Vitis (Schw.) E. \& E.-On stems of Vitis bicolor at Orient; determined by Prof. Dearness.

Gloniopsis Lathami Fairman-"On dead stems of Helianthus giganteus, Orient, N. Y., May 12, I918, Roy Latham, no. I194." A new species, described by Dr. Fairman in Proc. Rochester Acad. Sci. 6: 129. April 1922.

Gloniopsis Lathami asymetrica Fairman-"On dead stems of Lilium canadense, Orient, N. Y., May I2, I9I8, Roy Latham." A new variety, described by Dr. Fairman in Proc. Rochester Acad. Sci. 6: I29-I30. April 1922.

Hypocrea patella C. \& P.--On oak branches on the ground in dry woods at Cutchogue; determined by Dr. Lloyd. Mycol. Notes 65: 1077. Nov. 1920, Note 99I. Hypocrea patella "is a fairly common species around Cincinnati. While there is no doubt of the determination, I do not like the term 'bright yellow' as applied 
to it. It is rather orange yellow or antique brown of Ridgway to my eye."

Hypoxylon commutatum Nitschke-Orient, on branches of peach, Prunus Persica; determined by Prof. Dearness.

Iypoxylon Hoveianum Pk.-Cireenport on branches of Betula lenla; determined by Prof. Dearness.

IIypoxylon marginalum (Schw.) Berk.-Citrdiner's Island and Suthold on Fagus grandifolia; determined by Prof. Dearness.

IIysterium Prostii Duly-On trunk of Barchuris halimifoliu at Orient; determined by Prof. Dearness.

Hystcrographizm praclongum (Schw.) E. \& E.-On stems of Rosa blanda at Orient; determined by Prof. Dearness.

Hysterographium Smilacis (Schw.) E. \& E.-On stems of Similax rotundifolia at Orient; determined by Prof. Dearness.

Mclanomma curyophagum (Schw.) Sacc.-On hickory nuts, Orient; as reported by Dr. Fairman in Proc. Rochester Acad. Sci. 6: 101. Sept. I92I. Previously reported as Trematosphaeria nuclearia (DeNot.) Sacc.

Nummularia Bulliardi Tul.-On trunk of Quercus zelutina at East Marion; determined by Prof. Dearness.

Rosellinia aquila (Fr.) DeNot.-Greenport on trunk of Cary'a glabra; determined by Prof. Dearness.

$X$ ylaria brasiliensis (Theiszen)-On earth in a cornfield at Greenport in September. Determined by Dr. Lloyd who says; "We considered and figured this (Mycol. Notes no. 6I : 893, Fig. I559. Oct. 1919) from Brazil, but hardly expected it to come from New York. Surely it is the same plant, the features and habitat-growing in the ground with long rooting base, the simple clubs, the protruding small perithecia and the small spores, $4 \times 6 \mu$. The spores are smaller than the brazilian plant which measures up to $4 \times 8 \mu$. Sometime ago we received a lot of Xylaria from Carlos E. Chardon, Porto Rico, and one that although immature, we referred to Xylaria brasiliensis. We overlooked the label. This was collected by Prof. H. H. Whetzel at Ithaca, and hence Mr. Latham's collection is the second made in the United States. It is a rare find and a fine collection."

\section{MELANCONIALES}

Didymosporium propolidioides Fairman- "On old decorticated cedar (Juniperus) stump, Orient, N. Y., May I9I7, Roy Latham, no. 852." A new species described by Dr. Fairman in Proc. Rochester Acad. Sci. 6: 124. April 1922.

Melanconium sphaerospermum (Pers.) Link-On old stems of bamboo, Bambusa at Orient; determined by Dr. Fairman.

Pestaloz ia nucicola E. \& E.-On hickory nuts (Carya), Orient; determined by Dr. Fairman and reported in Proc. Rochester Acad. Sci. 6: 88. Sept. I921. 


\section{SPHAEROPSIDEAE}

Ascochyta Alismatis (Oud.) Trail-On leaves of Alisma Plantago-aquatica at Greenport; determined by Prof. Dearness. (Ascochyla Alis matis E. \&. E.)

Leptostroma Mitchellae Fairman-"On dead stems of Mitchella repens L., Orient, N. Y., May 1916, Roy Latham." A new species de. scribed by Dr. Fairman in Proc. Rochester Acad. Sci. 6: 123. April 1922.

Leptostroma Smilacis Cke.-Orient on stems of Smilax rotundifolia; determined by Prof. Dearness.

Phomopsis Arctii (Lasch.) Trav.-On stems of Arctium minus at Orient; determined by Dr. Fairman.

Phomopsis sp.-In the note under the description of the new species, Phomopsis rubiseda Fairman, from Lyndonville, N. Y., in Proc. Rochester Acad. Sci. 6: II8. April 1922, Dr. Fairman says: "Mr. Roy Latham sends a Phomopsis from Orient, N. Y., no. 425 collected on Rubus phoenicolasius Maxim, April 18, 1915, which has pycnidia 65-100 $\mu$ in diam. and spores 9-12 $\times .05^{-}$ I $\mu$ borne on slender hamate sporophores $20-24 \mu$ long. On March I6 1916, Mr. Latham collected his no. 8II at Orient, N. Y., on Rubus procumbens Muhl., which proves to be another Phomopsis with fusoid, guttulate, hyaline spores 6-7 $\times 1.5^{-}$ $2 \mu$ which seems referable to Phomopsis vepris (Nitschke) Trav., but the Orient specimens have smaller pycnidia and more slender spores. Cultural studies are needed to clear up the variability in the species of Phomopsis on Rosaceae."

Phyllosticta Lycii Ell. \& Kell.-On leaves of Lycium halimifolium at Orient; determined by Prof. Dearness.

Phyllosticta Staticis Petrak-On Limonium carolinianum at Orient; determined by Dr. Fairman.

Septoria Atriplicis Desm.-On leaves of Atriplex patula, var. hasiata at Orient; determined by Dr. Fairman.

Septoria atropurpurea Pk.-On leaves of Aster macrophyllus at Mattituck: determined by Prof. Dearness.

Septoria Lycopersici Speg.-On leaves of tomato, Lycopersicon esculentum, common; determined by Prof. Dearness.

Septoria Sii Rob. \& Desm.-On leaves of Sium cicutaefolium, common; determined by Prof. Dearness.

Septoria Trichostematis Pk.-On leaves of Trichostema dichotomum at Laurel; determined by Prof. Dearness, who says; "I think, this must be rather rare. Peck's first finding was in 1888 at Manor, Long Island."

Septoria Verbenae Rob. \& Desm.-On leaves of Verbena urticaefolia at Southold; determined by Prof. Dearness.

Sphaeropsis cerasina Pk.-On Prunus serotina at Orient; determined by Prof. Dearness.

Sphaeropsis Opuntiae Fairman--" On Opuntia Opuntia, Orient, N. Y., June 1919, Roy Latham, no. I807 in part." A new species described 
by Dr. Fairman in Proc. Rochester Acad. Sci. 6: 120. April 1922.

Vermicularia dematium (Pers.) Fr.-The Vermicularia common on herbaceous stems goes by the name $V$. dematium. "What appears to be the same thing was collected on hickory nuts at Orient, Long Island, N. Y., by Roy Latham in 1919. Mr. Latham's specimens have setae $120-250 \mu$ in height, and fuscoid, curved spores measuring 20-27 $\times 2.5-3 \mu . "$ Other specimens of this species on hickory nuts in central New York, have setae 7o$165 \times 6-7 \mu$; and spores $17-24 \times 3.5-4 \mu$. Determined and reported by Dr. Fairman in Proc. Rochester Acad. Sci. 6: 83 . Sept. 192 I.

Vermicularia liliacearum Schw.-Greenport on stems of Lilium canadense; determined by Dr. Fairman.

\section{USTILAGINACEAE}

Melanopsichium austro-americanum (Speg.) G. Beck-Common in cultivated fields on Polygonum Persicaria at Mattituck; determined by Dr. H. S. Jackson.

\section{PUCCINIACEAE*}

Polythelis fusca (Pers.) Arth. Greenport on Anemone quinquefolia, May.

Puccinia Antirrhini D. \& H.-Cireenport on garden snapdragon, Antirrhinum majus.

Puccinia Cyperi Arth.-On Cyperus Grayii at Bay View.

Puccinia Majanthae (Schw.) A. \& H.-On Polygonatum biflorum at Orient.

Puccinia obtecta $\mathrm{Pk}$.-Common on Scirpus americanus.

Fuccinia Pammelii (Trel.) Arth.-Greenport on Panicum virgatum; determined by Prof. Dearness.

Puccinia Polygoni-amphibii Pers.-On Geranium maculatum, Gardiner's Island, May 8, 1922. Dr. Arthur says: "The Geranium rust which has its alternate forms on different species of Polygonums is a common, wide-spread rust; but this aecial form which you send has not before been reported from your vicinity. It is known from the coast of Delaware and from further inland all along the Atlantic states; but has not been reported from Long Island."

Puccinia Seymouriana Arth.-Orient on Spartina glabra, var. alterniflora, October: "a new host for this species."

\section{TREMELLACEAE}

Hormomyces aurantiaca Bon.-Greenport on wood of Ilex verticillata; determined by Dr. Lloyd. "It has curious spores and is to be cfr. with Mycol. Notes, p. 7I2, fig. I066."

* Unless otherwise stated, the Rusts were determined by Dr. J. C. Arthur and are preserved in the Herbarium of Dr. Arthur at Purdue University, Lafayette, Indiana. 


\section{DACRYOMYCETACEAE}

Dacryomyces minor Pk.--On old wood at Orient; determined by Dr. Lloyd, who says, "I refer it to the above with doubt, but it appears to answer the description. It is a small ( $\mathrm{I} \mathrm{mm}$.) cushion shape, orange-yellow gelatinous plant, not changing much in drying. If consistently referred, its recent reference to Dacryomyces deliquescens is an error. It differs as noted above. But the structure is most puzzling and I think it is no Dacryomyces. The hymenial tissue is made up of branched septate hyphae (?) filled with granular matter septate and easily disarticulated into cylindrical hyaline guttulate spores (?). The sections have all the appearance of Dacryomyces spores. I find no basidia, although the forked hyphae (?) have much the appearance of Dacryomyces basidia."

\section{THELEPHORACEAE}

Peniophora laevigata Fr.-Bark of red cedar, Juniperus virginiana at Orient; determined by Dr. E. A. Burt, who says, "your specimen affords the first station for this species in the United States: I received a gathering several years ago from Canada."

Stereum albobadium (Schw.) Fr.-Old stems of Brassica oleracea gemmifera (Brussels sprouts), "Orient, R. Latham (in Mo. Bot. Gard. Herb., I 7267)," reported by Dr. Burt in Ann. Mo. Bot. Gard. 7: 2 18. Apr.-Sept. 1920.

\section{HYDNACEAE}

Hydnum friabile Fr.-On earth in dry woods, Cutchogue; determined by Dr. Lloyd and reported in Mycol Notes 65: 1077. Nov. 1920. A species similar to Hydnum pulcherrimum B. \& C. (H. septentrionale Fr.)

(To be Continued.) 



\title{
FLORA OF THE TOWN OF SOUTHOLD, LONG ISLAND
}

\author{
Stewart H. Burnham and Roy A. Latham \\ Third Supplementary List, Part 2*
}

\section{POLYPORACEAE $\uparrow$}

Cyclomyces Greenii Berk.-Earth in low woods at Cutchogue; reported in Mycol. Notes 65: 1077. Nov. 1920.

Fomes applanatus (Pers.) Fr.-On trunks of Baccharis halimifolia at Orient; reported as Fomes leucophaeus Mont. in Mycol. Notes 65: 1077. Nov. 1920.

Fomes conchatus (Pers.) Fr.-On trunks of Baccharis halimifolia at Orient; reported in Mycol. Notes 65: 1077. Nov. I920.

Fomes connatus Fr.-On trunks of Salix nigra at Southold.

Polyporus epileucus Fr.-On Quercus velutina at Greenport; Dr. Lloyd says "a rare species."

Polyporus galactinus Berk.-Cutchogue on old wood.

Polyporus rutilans (Pers.) Fr.-On Quercus velutina at Greenport.

Polyporus Schweinitzii. Fr.-Southold on coniferous wood.

Polystuctus focicola B. \& C.-On earth in dry woods, Southold; determined by Dr. Lloyd, who says; "We refer this to Polystictus focicola on its large pores, although in reality it is a 'new species.' It is a large pored form of Polystictus cinnamomeus with bright cinnamon color, while Polystictus focicola proper is a large poroid form of Polystictus perennis with dull color."

* Part one of this list was published in Torreya, Vol. 23, No. I, Jan.-Feb. I923.

$\dagger$ The Polypores were determined by Dr. C. G. Lloyd and are preserved in the Herbarium of the Lloyd Museum and Library at Cincinnati, Ohio. 
Trameles pusilla Lloyd.-Creenport on Quercus alba; determined by $\mathrm{Mr}$. Lloyd, who says: "This is the second collection I have received. The original from Dr. Stoker, Minnesota, was publisned and figured on page 774 (of Mycol. Notes, no. 54). We described the pores as white, and so they are on fleshy dried specimens, but on these and on Dr. Stocker's specimens now they have turned reddish."

\section{AGARICACEAE}

Cantharellus carbonarius A. \& S.-On earth in woods, Southold; determined by Dr. Lloyd.

Flammula sapinea Fr.-On trunk of Quercus alba in a swamp at Southold; determined by Dr. Lloyd.

Lentinus tigrmus (Bull.) Fr.-Rotten log of Quercus velutina, Cutchogue; reported by Dr. Lloyd in Mycol. Notes 65: 1077. Nov. 1920, who says, "These are the first specimens we have received that are not parasitized."

Marasmius fagineus Morg.-Orient; determined by Dr. Lloyd.

\section{GASTEROMYCETES}

Cyathus striatus (Huds.) Willd.-Orient; reported by Dr. Lloyd, Mycol. Notes 65: 1077. Nov. 1920.

Ithyphallus rubicundus (Bosc) Ed. Fisch.-Moore's woods in rich soil, Greenport, July 25, 1920; reported by Dr. Lloyd in Mycol. Notes 65: 1077. Nov. 1920, as Phallus rubicundus. Dr. Lloyd says: "Mr. Latham found but a single specimen, but the finding of the plant so far north is noteworthy as illustrative of exceptional northern distribution of tropical species. It is rare in our southern States and I believe has heretofore only been found in Florida. Mr. Latham s plant is Phallus gracilis as illustrated in the Phalloid Synopsis, Fig. 6, but as there stated it is only a slender form of Phallus ruticundus and the name gracilis should be dropped. It is the only one of the genus Phallus that is red."

Lysurus borealns (Burt) P. Henn.-On earth in cornfields; Orient and Greenport. Dr. Lloyd says: "You will find many references to this in my writings for it is a Phalloid not known to us 20 years ago. It was named Anthurus borealis by Burt but it is a Lysurus and probably same as Lysurus australiensis of Australia."

\section{HEPATICAE}

Calypogeia sphagnicola (Arn. \& Perss.) Warnst. \& Loeske.-Wet sandy soil at Laurel; determined by Dr. G. H. Conklin.

Lepidozia setacea (Web.) Mitt.-On wet, sandy bank at Laurel; determined by Dr. Conklin. 


\section{MUSCI*}

Brachythecium acuminatum (Hedw.) Lindb.-Greenport, base of oak trees in wet woods.

Brachythecium plumosum (Sw.) B. \& S.-Gardiner's Islano.

Corriphyllum Boscii (Schwaegr.) Grout.-Earth in dry woods at Laurel.

Ditrichum tortile (Schrad.) Hampe.-Wet sandy bank at Laurel.

Fontinalis Lescurii Sull.-Mattituck in water in a swamp.

Philonotis fontana (L.) Brid.-Gardiner's Island on earth in wet woods.

Polytrchum commune L., var. perigoniale (Mx.) B. \& S. - In dry woods at Southold.

Sphagnum capillaceum (Weiss) Schrank, var. tenellum (Schimp.) A. L. Andrews.-Mixed with Sphagnum palustre.

Sphagnum fimbriatum Wils.

Sphagnum imbricatum Hornsch., var affine (Ren. \& Card.) Warnst.-Laurel. Sphagnum palustre L.

Sphagnum subsecundum Nees-Gardener's Island in wet woods. No. 3562 . "A form of S. subsecundum in the broad sense. . for those who separate it into a number of species; it corresponds nicely with Sphagnum auriculatum Schimp."

\section{PTERIDOPHYTA}

\section{POLYPODIACEAE}

Athyrium thelypteroides (Mx.) Desv.-Orient; determined by Dr. F. W. Pennell.

\section{SPERMATOPHYTA}

Picea rubens Sarg.--The young seedlings of Gid's Island were destroyed by a fire during the spring of 1922 .

Potamogeton epihydrus Raf.-Laurel in shallow water; determined by Dr. Pennell.

Alopecurus aristulatus Michx.-Orient in waste places; determined by Mrs. Agnes Chase.

Danthonia compressa Austin.-Southold, common in dry woods, plants reaching 3 feet in height; determined by Mrs. Chase.

Eragrostis cilianensis (All.) Link.-Sandy cultivated fields at Bay View and Laurel; determined by Mrs. Chase and at the New York Botanical Garden. (Eragrostis major Host.; E megastachya (Koeler) Link.)

Festuca Shortii Kunth.-Wet woods, Mattituck; determined by Mrs. Chase. (Festuca obtusa Spreng.)

Pancium barbulatum Mx.- Southold in wet sandy soil; determined by Mrs. Chase. In the preliminary list, this was included with Panicum microcarpon Muhl. as Panicum barbulatum Nash. Mrs. Chase, in her recent list, listed these plants as different species.

* The mosses were determined by Mr. G. B. Kaiser and deposited in the Herbarium of the Sullivant Moss Society; except the Sphagnums which were determined by Dr. A. L. Andrews. 
Panicum Boscii Poir, van. molle (Vasey) Hitchc. \& Chase.-Dry woods at Cutchogue; determined by Mrs. Chase.

Panicum psendopubescens Nash-1)ry woods, ('utchogne; determined by Mrs. Chase.

Phalaris canariensis L.-Border of wools, Greenport (( irant Sterling); determined by Mrs. Chase.

Spartina cynosuroides (L.) Roth.-High loorders of salt marshes at Bay View, plants 8 feet tall; determined by Mrs. Chase,

Spartina patens (Ait.) Muhl., var. caespitosa (Eaton) Hitchc.-High borders of salt marsh at Orient, in clumps, 3 feet tall; determined by Mrs. Chase.

Carex pennsylvunica Lam., var. lucorum (Wilkl.) Fernald.--Sonthold in dry woods, May; determined by Mr. G. P. VanEseltine.

Citrex vuriu Muhl., var. colorata Bailey.-Sonthold in wet sandy soil, May; determined by Mr. Van Eseltine.

Scirpus campestris Britton, var. paludosus (A. Nels.) Fernald.-Bay View, no. 3690 ; determined by Dr. Pennell.

Sisyrinchium arenicola Bicknell.-Cutchogue, sandy horders of a salt marsh, common; determined by Dr. Pennell.

Ilabenaria ciliaris (L.) R. Br.-A colony of several dozen plants in low ground at Greenport (Grant Sterling).

Spiranthes Beckii Lindl.-Southold in sandy s jil; determined by Mr. Pennell.

Ulmus americana L.-Gardiner's Island, May 6-8, I92I, in fruit; verified by $\mathrm{Mr}$. Norman Taylor. The second Long Island record.

Rumex Britannica L.-Mattituck, no. 3695; determined by Dr. Pennell.

Polygonum exserlum Small.-Salt marshes, Cutchogue; determined by Dr. J. K. Small.

Kochia Scoparia (L.) Roth.-Orient, roadside and waste places; determined at the N. Y. Botanical Garden.

Oxybaphus linearis (Pursh) Robinson.-Dry sandy beaches at Orient; determined at the N. Y. Botanical Garden.

Silene conica L. - A weed in sandy fields at Laurel and Cutchogue, no. 34 77; determined by Dr. Pennell, who says, "we have no specimen of this European species from the New World, nor is it included in any of our manuals."

Ranunculus repens L.-Gardiner's Island; determined by Mr. Taylor.

Akebia quintata Decaisne.-Escaped at Orient, no. 3+2 I; determined by Dr. Pennell.

Chelidonium majus L.-Waste places in woods, Southold (Mrs. M. A. Fay).

Sisymbrium Thalianum (L.) J. Gay.-Cutchogue in sandy fields; determined at the N. Y. Botanical Garden.

Ribes Grossulara L.-Dry woods, Laurel; determined at the N. Y. Botanical Garden.

Agrimonia striata Mx.-Greenport in dry woods; determined at the N. Y. Botanical Garden.

Crataegus macrosperma Ashe.--Orient in low woods; determined by Mr. W. W. Eggleston.

Crataegus pruinosa (Wendl.) K. Koch.-Several small trees in dry woods at Greenport; determined by Mr. Eggleston. 
Crataegus straminea Beadle.-Orient in low woods, near salt marsh; determined by Mr. Eggleston. (Crataegus intricata Sarg.)

Rubus argutus Link.-Orient in low woods; determined at the N. Y. Botanical Garden.

Rubus Enslenii Tratt.-Mattituck in hilly woods; determined at the N. Y. Botanical Garden.

Rubus nigricans Rydb.-Southold in sandy soil, plants spreading on the ground in open fields; determined by Dr. P. A. Rydberg.

Cassia Chamaecrista L.-Southold, August I9I4 (H. E. Gordon); in the Herbarium of the N. Y. State College of Agriculture at Ithaca.

Desmodium laevigatum (Nutt.) DC.-Dry woods, Southold; determined at the Bureau of Plant Industry, Washington.

Lespedeza repens (L.). Bart.-Southold in dry woods.

Oxalis corniculata L.-Orient in rich woods, plants reaching 2 feet in height; determined by Dr. Pennell as Oxalis cymosa Small.

Ilex verlicillata (L.) Gray.-Specimens showing the variability of the species; determined by Dr. Pennell.

Evonymus europaeus L.-East Marion, border of woods and roadside (Mabel Wiggins); verified at the N. Y. Botanical Garden.

Hypericum boreale (Britton) Bicknell.-Gardiner's Island, no. 3869, rare; determined by Dr. Pennell.

Helianthemum dumosum (Bickn.) Fernald.-Dry open woods, Southold and Cutchogue, no. 4056. Dr. Pennell says, "a species characteristic of Nantucket and the Cape Cod country, but of which yours is our first specimen from Long Island."

Lechea minor L.-Cutchogue and Southold in dry woods; determined by Dr. Pennell.

Coelopleurum actaeifolium (Mx.) Coult. \& Rose.-Dry hills facing the Sound, Orient and East Marion; determined by Dr. J. N. Rose. Mr. Latham wrote Dr. Rose, April I92I, as follows. "The specimens were not very good as they were taken late in December while collecting birds. The plants were quite common along the top of the Sound bank in high rocky dry ground. At that date, the basal leaves were green, and the dry fruiting stalks were from 4-6 feet high. It was a stout plant. Ligusticum scoticum is a very common plant at the borders of salt marshes in Orient. It is different from this plant; it is not as stout, nor so tall and never grows in such high dry grounds." Mr. Latham again collected it in June I92I, and wrote Dr. Rose as follows. "I am sending you today specimens collected the past summer at Orient. There is quite a colony of this species growing on a high bank of the Sound coast and about three miles east another small colony on a low beach between the Sound and Bay." Dr. A. W. Evans in Torreya, June 1917, reported Coelopleurum actaeifolium from Fisher's Island, the only other station known further south than Nantucket, Massachusetts.

Cornus alternifolia L. f.-Gardiner's Island, probably introduced; determined by Dr. Pennell. 
IIypopitys insignata Bicknell.-Frequent in rich woorls at Greenport, Southold, Peconic and Cutchogue. Plants highly colored, red or crimson. Dr. Pennell says: "This bright-flowered plant of the early fall was described in the Torreya Bulletin for August 19I4, from Martha's Vineyard; it is certainly a brilliant species."

Vaccinium vacillans Kalm, var. crinilum Fernald.-Dry woods, Laurel; determined by Dr. Pennell.

Buddleia Daridii Franch.-Escaped, border of swamp) at Southold; determined at the N. Y. Botanical Garden.

Lamium purpureum L.-Orient in cultivated fields and waste places; determined at N. Y. Botanical Garden.

Monarda fistulosa L.-Orient in rich woods.

Salvia pratensis L.- In fields and pastures at Orient, frequent before the war, now disappearing with the plowing of old fields. No. 3422 . Determined by Dr. Pennell, who says, "Your plant is smaller. flowered than any of our eastern specimens; but I think it must be a form of this species."

Salureja vulgaris (I..) Fritsch.-Southolk, roadsides, rare (Mrs. F. R. Mitchell); Gardiner s Island (E. S. Miller), reported as Calamintha Clinopodrum Benth., in Bull. Torr. Bot. Club 7: 18. Feb. 1880.

Veronca americana Schwein.-Greenport in wet woods; determined at $\mathrm{N}$. Y. Botanical Garden.

Hicracium praealium Vill-Sandy beaches at Orient, rare; determined at N. Y. Botanical Garden.

IIicracium pratense Tausch.-Greenport in fields; determined at N. Y. Botanical Garden.

Prenanthes altissima L.-Mattituck in dry woods and sandy beaches; determined at N. Y. Botanical Garden.

Achillea Ptarmica L.-Orient in fields and waste places; determined by Dr. Pennell.

Aster Schreberi Nees.-Dry woods, Southold, basal leaves reaching 8 inches in length; determined by Dr. Pennell.

Centaurea cineraria L.-Waste places, rare, Orient; determined at N. Y. Botanical Garden. (Centaurea candidissima Lam.)

Centaurea paniculata 1.--Roadsides, locally common, Bay liew; determined at N. Y. Botanical Garden.

Chrysanthemum Parthenium (L.) Bernh.-Occasional in waste places and fields.

Corıopsis lanceolata L. - Southold in dry pastures, a rare escape; determined at N. Y. Botanical Garden.

Eupatorium urticacfolum Reichard.-Dry woods, Southold; determined by Dr. Pennell.

Helenium nudiflorum Nutt.-Sandy swamp at Southold; determined at $\mathrm{N}$. Y. Botanical Garden.

Solıdago puberula Nutt.-Dry open woods at Laurel; determined at N. Y. Botanical Garden. 
The total number of species recorded in the preliminary, the first, the second and the third supplementary lists is 246r.

The number of Insect Galls, 93; Slimemolds, 15; Algae, Ior ; Fungi, 813; Lichens, I26; Hepatics, 31 ; Mosses, 106; Ferns, 36; Flowering Plants, I I 3 \%. 

Cudoniella marcida (Müll.) Sacc.-The note in the Third Supplementary list was published in Dr. C. G. Lloyd's Mycol. Notes 68: II82, fig. 2387. Jan. 1923 .

Helvella elastica Bull.-Earth in dry woods at Mattituck; determined by Dr. C. G. Lloyd.

Leotia chlorocephala Schw.-Earth in dry woods at Laurel; determined by Dr. Lloyd and reported in Mycol. Notes 69: I188. July 1923.

Orbilia vinosa (A. \& S.) Karst. Dead twigs in a swamp at Laurel; determined by Dr. Chas. E. Fairman.

Phialea Aspegrenii (Fr.) Gill,-O-Old twigs in rich woods at Mattituck; determined by Prof. Dearness.

Pyrenopeziza subatra (C. \& P.) Sacc.-On Solidago at Orient; determined by Prof. Dearness.

\section{PYRENOMYCETES}

Caryospora Putamium (Schw.) DeNot.-On peach, Prunus Persica pits at Orient; determined by Dr. Fairman.

Chaetomium elatum Kuntze-On Quercus velutina at Orient; determined by Prof. Dearness.

Diaporthe Baccharidis (Cke.) Sacc.-On Baccharis halimifolia; determined by Prof. Dearness.

Diaporthe oncostoma (Duby) Fckl.-On Amelanchier canadensis at Orient; determined by Prof. Dearness.

Diatrype asterostoma B. \& C.-On Amelanchier at Orient; determined by Prof. Dearness.

Diatrype bullata (Hoffm.) Fr.-On Acer platanoides at Orient; determined by Prof. Dearness.

Diatrype fibritecta C. \& E.--Orient on dead branches of Juniperus virginiana lying on the ground; determined by Prof. Dearness, who says, "a fine thing."

Diatrype Maclurae E. \& E.-On Rhus Toxicodendron, var. radicans at Orient; determined by Prof. Dearness.

Erysiphe graminis DC.-On wheat, Triticum aestivum at Orient; determined by Prof. Dearness.

Eutypa ludibunda Sacc.-On Gleditsia triacanthos at Orient; determined by Prof. Dearness.

Eutypella constellata (B. \& C.) E. \& E.-Greenport on Carya glabra; determined by Prof. Dearness.

Eutypella glandulosa (Cke.) E. \& E.-On Ailanthus glandulosa at Mattituck; determined by Prof. Dearness.

Hypomyces chrysospermus Tul.-The conidial form Sepedonium on Boletus; determined by Dr. Lloyd and reported in Mycol. Notes 69: I I 88. July 1923.

Hysterium angustatum A. \& S.-Orient on twigs of Crataegus Crus-galli; determined by Prof. Dearness.

Hysterographium Mori (Schw.) Rehm, var. Gerardi C. \& P.-On Prunus serotina at Orient; determined by Prof. Dearness. 
Hysterographium Putamium (Cke.) Sacc-On pits of Prunus maritima at Southold; determined by Dr. Fairman, who says, "a rare species."

Lophodermium arundinaceum Chev., var. culmigenum (Fr.) Fckl.-Mostly Leptostromella hysterioides Sacc. On Triticum aestivum at Mattituck; determined by Prof. Dearness.

Melanconiella Decoraensis (Ellis) Sacc._- "Dead branches of Betula populifolia Marsh., Orient, Long Island, Roy Latham, March 1919. Determined by Dearness." N. Y. State Mus. Bull. 243-244: 85 . Issued May i 5, 1923.

Metasphaeria defodiens (Ellis) Sacc.-Stems of Juncus dichotomus, Southold; determined by Prof. Dearness, who says, "near this" species.

"The only record I know for this is Iona, N. J."

Phyllachora Agrostidis Orton-"On leaves of Agrostis alba. Orient, Long Island, Roy Latham, no. 610, October 18, 1914. Determined by Dr. C. R. Orton." N. Y. State Mus. Bull. 243-244: 91. Issued May i 5, I923.

Phyllachora puncta (Schw.) Orton-"Leaves of Panicum Wrightianum, Southold, Latham, no. 6II, November 4, I9I4. Determined by Dr. C. R. Orton." N. Y. State Mus. Bull. 243-244: 92. Issued May 15, 1923.

Phyllactinia corylea (Pers.) Karst.-Leaves of Betula populifolia at Greenport; determined by Prof. Dearness.

Physalospora thyoides (C. \& E.) Sacc.-On Juniperus virginiana at Orient; determined by Prof. Dearness.

Rosellina albolanata E. \& E.-On Quercus velutina; determined by Dr. Fairman.

Trematosphaeria perinsa (Pers.) Fckl.-Pits of Burbank plum, Prunus trifolia $\mathrm{x}$; determined by Dr. Fairman.

Valsa IIoffmanni Nke.-Greenport on Cralaegus Crus-galli; determined by Dr. Fairman.

Valsa leucostoma (Pers.) Fr.-Orient on Malus; determined by Prof. Dear. ness.

Valsa obtecta C. \& E.-On Clethra alnifolia at Laurel; determined by Prof. Dearness.

Xylaria apiculata Cke.-Bark of living oak at Greenport; determined by Dr. Fairman, who says, "According to Lloyd. In Rehm Ascomycetes, No. 1912, a similar thing is called $X$, arbuscula Sacc., var. biceps Speg."

Xylaria brasiliensis (Theiszen) - The note in the Third Supplementary list was published in Dr. Lloyd's Mycol. Notes 68: 1 I 75, fig. 2338. Jan. 1923 .

\section{HYPOMYCETES}

Alternaria Solani (E. \& M.) Jones \& Grout-On Solanum tuberosum, common; determined by Prof. Dearness.

Cercospora Symplocarpi Pk.--On Symplocarpus foetidus at Greenport; determined by Prof. Dearness.

Cercospora varia $\mathrm{Pk}$.-On Viburnum dentatum at Greenport; determined by Prof. Dearness. 
Cladosporium epimyces Cke.-On Pleurotus ostreatus at Orient; determined by Prof. Dearness.

Helminthosporium obclavatum Sacc.-Orient on Juniperus virginiana; determined by Prof. Dearness.

Heterosporium gracile (Wallr.) Sacc.-Orient on Iris; determined by Prof. Dearness.

Isaria farinosa (Dicks.) Fr.-On pupa in rich woods at Mattituck. Determined by Dr. Fairman, who says, "It is probably the var. prolifero-ramosa of Saccardo, though I have not seen this and have no recora of its finding in this country."

Ramularia Plantaginis Ell. \& Mart.-Orient on Plantago Rugeili; determined by Prof. Dearness.

Septocylindrium aromaticum Sacc.-Southold on Acorus Calamus; determined by Prof. Dearness.

Stilbum erythrocephalum Ditm.-Orient on droppings of chickens; determined by Dr. Fairman.

Trichothecium roseum (Pers.) Link-On stems of Akebia quinata at Orient; determined by Prof. Dearness.

Tubercularia rosea Schreb.-Illosporium roseum (Schreb.) Mart. Orient on pine wood. Determined by Dr. Lloyd, who says, "Tubercularia rosea from Roy Latham, New York. We name this only as a guess or rather two guesses. First, that it answers the 'description,' habits, etc., of Dacryomyces roseus as named by Fries, and second, that Dacryomyces roseus is really a Tubercularia. All these little gelatinous plants were called Dacryomyces or Tremella by the old namers who did not examine them with the microscope. It is a very small rose-colored tremellaceous cushion growing on a mossy substratum which fits 'Dacryomyces roseus.' That is about all I think anyone knows about it." Reported in Mycol. Notes 69: I2II. July I923 as "Note II 80. ."

\section{MELANCONIALES}

Gloeosporium fructigenum Berk.-Old fruit of Pyrus communis at Orient; determined by Prof. Dearness. A stage of Glomerella cingulata (Atks.) S. \& S.

Myxosporium subviride E. \& E.-Greenport on Betula; determined by Prof. Dearness.

\section{SPHAEROPSIDEAE}

Aposphaeria nucicola E. \& E.-Orient on nuts of Carya glabra; determined by Dr. Fairman.

Aposphaeria Putamium (Speg.) Sacc.-Pits of German prune, Prunus domestica at Orient; determined by Dr. Fairman.

Ascochyta Dianthi (A. \& S.) Berk.-Orient on Dianthus Armeria; determined by Prof. Dearness.

Ascochyta Philadelphi Sacc. \& Speg.-On Philadelphus coronarius, Laurel, no. II 37 ; determined by Dr. Fairman, who requested that the 
following notes be published. "Ascochyta Philadelphi Sacc. \& \& Speg.? Pycnidia scattered, subepidermal becoming erumpent, globose depressed, centrally ostiolate, of delicate pseudo pycnidial structure, brown or black, about $200 \mu$ in diam.; spores numerous, ellipsoid, rounded at the ends, at first continuous and unconstricted, becoming with age uniseptate and slightly constricted at the middle, greenish hyaline, $7.5 \times$ $3.5 \mu$. On living or languishing stems of Philadelphus sp. Laurel, N. Y. This is probably the stem form of the above species. The specimens are in a young condition and show mostly Phoma-like spores."

Ascochyta Pisi Lib.-Mattituck on Vicia; determined by Prof. Dearness.

Camarosporium Berkleyanum (Lev.) Sacc.-On Ailanthus glandulosa, Mattituck; determined by Prof. Dearness. Camarosporium subfenestratum (B. \& C.) Sacc. of some botanists.

Dinemasporium hispidulum (Schrad.) Sacc., var. herbarum Cke--Stems of Thalictrum revolutum at Orient; determined by Prof. Dearness.

Discosia Artocreas (Tode) Fr. (Discosia faginea Lib.)-Greenport on leaves of Fagus grandifolia; determined by Prof. Dearness.

Discula Platani (Pk.) Sacc.-Orient on branches of Platanus occidentalis; determined by Prof. Dearness.

IIaplosporella Bignoniue (Schw.) Starb.-On Tecoma radicans at Orient; determined by Dr. Fairman.

IIaplosporella Dulcamara Dearn. \& House- "On dead stems of Solanum Dulcamara L., Sandlake, Rensselaer County, Dr. C. H. Peck (Type), year not indicated. Orient, Long Island, Roy Latham, 1916. .... N. Y. State Mus. Bull, 243-244: 75. Issued May $15,1923$.

Ilendersonir Desmazieri Mont.--Orient on Plıtanus occidentulis; determinerl by Prof. Dearness.

Leptostroma herbarm (Fr.) Link-Iaurel on Dioscorea villosa; determined by Prof. Dearness.

Leptosiroma Pinastri Desm.--Needles of Pinus rigida and P. Strobus, throughout the region; determined by Prof. Dearness.

Phoma glandicola (Desm.) Ler.-On insect galls on Quercus alba at Greenport; determined by Dr. Fairman.

Phoma Mariae Clinton-Orient on Lonicera japonica; determined by Prof. Dearness.

Phoma subcircinata E. \& E.-Pods of Phaseolus lunatus at Cutchogue; determined by Dr. Fairman.

Phoma Syingae B. \& C.-Orient on Syringa vulgaris; determined by Prof. Dearness.

Phomopsis depressa (Lev.) Trav.-Orient on Syringa vulgaris; determined by Dr. Fairman.

Phomopsis tpicarpa Sacc.-Pods of Robinia Pscudo-Acacia at Orient; determined by Dr. Fairman.

Phyllosticta A maranthi Ell. \& Kell.-Laurel on Acnuda tubcrculata; determined by Prof. Dearness, who says that he is "not aware that this has been collected on Acnida before." 
Phyllosticta Lappae Sacc.-Orient on Arctium minus; determined by Prof. Dearness.

Phyllosticta latifoliae E. \& E. - Greenport on Kalmia latifolia; determined by Prof. Dearness.

Phyllosticta Nyssac Cke.-Greenport on Nyssa syliatica; determined by Prof. Dearness.

Phyllosticta smilacina (Pk.) Dearn.-(Sphaeropsis smilacina $\mathrm{Pk}$.$) -On leaves$ of Smilax at Southold and Orient; determined by Prof. Dearness.

Septoria carpogena E. \& E.--Old fruit of Celtis occidentalis at Mattituck; determined by Dr. Fairman.

Septoria Celtis-gallae Gerard-Old galls on Celtis occidentalis at Mattituck; determined by Dr. Fairman.

Septoria lepidiicola Ell. \& Mart.-On Lepidium at Orient; determined by Prof. Dearness.

Sphaeronema clethrincolum Ell.-Greenport on Clethra alnifolia; determined by Prof. Dearness.

Sphaeropsis Betulae Cke, var. lutea Dearn. \& House-On Betula populifolia at Orient; determined by Prof. Dearness. The variety on dead branches of Betula lutea is described in N. Y. State Mus. Bull.

243-244: 79. Issued May I5, I923.

Sphaeropsis Cydoniae C. \& E.-Orient on Cydonia japonica; determined by Prof. Dearness.

Sphaeropsis pericarpii $\mathrm{Pk}$. - On nut of Carya glabra at Orient; determined by Dr. Fairman.

Sphaeropsis Sassafras C. \& E.-Orient on Sassafras; determined by Prof. Dearness.

Sphaeropsis simillima $\mathrm{Pk}$.-On Acer rubrum and Acer platanoides at Orient; determined by Prof. Dearness.

\section{USTILAGINACEAE}

Ustilago sphaerogena Burr.-Orient on Echinochloa Walteri; determined by Dr. H. S. Jackson.

\section{PUCCINIACEAE}

Aecidium Apocyni Schw.-On Apocynum androsaemifolium at Mattituck; determined by Dr. Jackson.

Cronartium cerebrum (Pk.) Hedgc. \& Long-Peconic on Pinus rigida; determined by Prof. Dearness.

Cronartium comptoniae Arth.-Southold on Pinus rigida; determined by Dr. Jackson.

Earlia speciosa (Fr.) Arth.-Cutchogue on Rosa blanda; determined by Dr. Jackson. (Phragmidium speciosum (Fr.) Cke.)

Puccinia Andropogonis Schw.-On Chelone glabra at Greenport; determined by Dr. Jackson.

Puccinia angustata $\mathrm{Pk}$.-On Lycopus virginicus at Greenport; determined by Dr. Jackson.

Puccinia asperifolii (Pers.) Wettst.-On Secale cereale, common; determined by Dr. Jackson. 
Puccinia Asterum (Schw.) Kern-Orient on Dulichium arnndinaceum; determined by Prof. Dearness.

Puccinia Fraxinata (Link) Arth.-On Fraximus americana at Greenport and Southold; determined by Dr. Jackson.

Puccinia Hibisciatum (Schw.) Kell.-Orient on Muhlenbergia sylvatica; determined by Dr. Arthur.

Puccinia Iridis (DC.) Wallr.-Common on Iris versicolor at Orient and Greenport; determined by Dr. Arthur.

Puccinia pustulatum (Curtis) Arth.-Orient on Andropogon scoparius; determined by Prof. Dearness.

Uredinopsis mirabilis (Pk.) Magnus-"II, II I, on fronds of Onoclea sensibilis L., Gardiner's Island, Roy Latham, August igr9. On fronds of Lorinseria areolata (L.) Presl., [Woodwardia areolata] at Greenport, Latham, August 1920. The aecial stage (Peridermium balsameum Peck) occurs on Abies balsamea (L.) Mill., a host tree, not known to occur on Long Island. It has been collected by Peck on Anchistea rirginica (L.) Presl., [Woodwardia virginica] at Manor, Long Island." N. Y. State Mus. Bull. 243-244: 85. Issued May 15, 1923.

Uromyces Iledysari-paniculati (Schw.) Farl._-"On leaves of Meibomia Dillenii (Darl.) Ktze., [Desmodium Dillenii] at Bay View, town of Southold, Long Island, Roy Latham, August 2I." (Nigredo Hedysari-paniculati (Schw.) Arth.). N. Y. State Mus. Bull. 243-244: 85. Issued May I5, 1923.

Uromyces seditiosus Kern-Laurel on Aristida tuberculosa; determined by Dr. Jackson who says, "new to New York state." Also determined by Prof. Dearness.

\section{TREMELLACEAE}

Tremella lutescens Pers.-Old wood at Orient; determined by Dr. L.loyd.

\section{DACRYOMYCETACEAE}

Dacryomyces deliquescens (Bull.) Duby-On Juniperus virginiana at Orient; determined by Dr. Lloyd. "Note II8I-Dacryomyces deliquescens from Roy Latham, New York. This soaks out pale yellow and is Dacryomyces deliquescens of American mycology. This collection is the conidial condition and has abundant small globose, hyaline spores about 4 mic in diameter and borne in chains. It has developed since I have been in Europe and Buller's second volume has appeared that this is not Dacryomyces deliquescens of English tradition and of Buller's book. The curious oidium spores of the English plant which are first shown in Buller's book to be on separate bodies from the basidia bearing spore bodies are entirely different from these spores. I do not believe we have the English plant with us, though both countries apply the name Dacryomyces deliquescens to their respective plants. In English tradition (started by Berkeley) this oidium form which is deep orange is called Dacryomyces 
stellatus 'Nees.' That is a taxonomic mistake, for the Neesian plant was shown with furcate basidia and it is the common species of Sweden better called Dacryomyces abietinus" Mycol. Notes 69: I2II. July 1923.

Dacryomyces minor $\mathrm{Pk}$ - The note in the Third Supplementary list was published in Mycol. Notes 67: II6I. July 1922, as "Note II30."

Dacryomyces sp.—"A minute Dacryomyces from Roy Latham, New York. Mr. Latham sends us decorticate wood, 'a very minute Tremellalike plant.' Mr. Latham has good eyes, for I cannot see it even with a hand lens, neither dried nor soaked. But a scraping under the microscope shows a definite little species of Dacryomyces, typical as to basidia and appearance, but no spores found. Its color is pale yellow. I am afraid it is too small to name, though formidable Latin names are applied to more minute plants." Reported by Dr. Lloyd in Mycol. Notes 69: I2 I8. July I923.

\section{THELEPHORACEAE}

Stereum lobatum (Kuntze) Fr.-Stereum versicolor Fr. of the Preliminary list and Stereum fasciatum Schw. of the First Supplementary list should be referred to this species.

\section{POLYPORACEAE}

Fomes lucidus (Leyss.) Fr.-On Acer rubrum at Greenport; determined by Dr. Lloyd, and listed in Mycol. Notes 69: II88. July I923.

Polyporus circinatus Fr.-Earth in dry woods at Mattituck; listed in Lloyd's Mycol. Notes 69: II88. July 1923.

Polyporus cristatus (Pers.) Fr.-Greenport on Quercus alba; listed in Lloyd's Mycol. Notes 67: II6I. July I922.

Polyporus Spraguei B. \& C.- "Note IIo9. Polyporus Spraguei from Roy Latham, New York. My first impression was that this was not Spraguei for I associate a hard, rigid context with the species and this is rather soft and crumbly. I do not find any other difference, however, and to eye it is exactly Spraguei." Lloyd's Mycol. Notes 67: II6I. July I922.

Polystictus focicola B. \& C.-The note in the Third Supplementary list was published in Mycol. Notes 67: II64. July 1922, as "Note II I 4."

Polystictus perennis (L.) Fr.-Greenport. Listed in Lloyd's Mycol. Notes 69: II 88. July I923.

Poria flavescens (Schw.) Cke.-Orient on Juniperus virginiana; determined by Dr. E. A. Burt.

Poria viticola (Schw.) Cke.-Orient on Platanus occidentalis and Greenport on Lyonia ligustrina; determined by Prof. Dearness.

Trametes protracta Fr.-Gardiner's Island on log of Pinus rigida; listed in Lloyd's Mycol. Notes 69: I I88. July I923.

Trametes pusilla I.loyd.--The note in the Third Supplementary list was published in Mycol. Notes 69: 1207. July 1923, as "Note I148." 


\section{AGARICACEAE}

Lenzites betulina (L.) Fr.-Greenport on Quercus stellata; determined by Dr. Lloyd who says, "Your specimen is what is called [Lenzites] flaccida (Bull.) Fr."

Mycena haematopa Berk.-Coniferous wood at Orient; determined by Prof. Dearness.

Pleurotus approximans Pk.-Orient on Baccharis halimifolia; determined by Prof. Dearness.

Pleurotus niger Schw.- "Note I I79. Pleurolus niger from Roy Latham, New

York. This would have been of much interest to me years ago, when I was working on agarics. I could never understand why I never found Plturotus niger while the similar little Pleurotus striatulus was so frequent. Pleurotus niger is truly a black plant with black gills and globose, hyaline 4 mic. spores. The gill margins have large white cystidia (or crystals perhaps)." Lloyd's Mycol. Notes 69: I2 I I. July 1923.

Psilocybe uda (Pers.) Fr.-Sphagnum at Greenport; determined by Prof. Dearness.

\section{LICHENES}

Blastenia ferruginea (Huds.) Arn., var. discolor (Willey), n. comb.-Orient on Juniperus virginiana, April 25, 1910 and January 9, I9II; determined by Dr. Bruce Fink. Reported in the Preliminary list as Lecanora (§Callopisma) ferrugineum discolor (Willey). (Placodium ferrugineum discolor Willey).

Ramalina Willeyi Howe-Orient on Juniperus virginiana, October 1, 1914; determined by Dr. R. Heber Howe, Jr.

\section{HEPATICAE}

Cephaloziella byssacea (Roth) Warnst.-Laurel; determined by Dr. Geo. H. Conklin.

\section{MUSCI}

Dicranum montanum Hedw.-Base of trees in swamp at Greenport; determined by Mr. Geo. B. Kaiser.

Eurhynchium rusciforme (Neck.) Milde-Gardiner's Island, rocks in a woodland stream; determined by Mr. Kaiser.

Orthotrichum Schimperi Hamm.-Orient on Robinia Pseudo-Acacia.

Orthotrichum strangulatum Sull, var.-Rock in woods at Gardiner's Island.

Platygyrium repens (Brid.) B. \& S.-Base of trees at Greenport; determined by Mr. Kaiser.

\section{PTERIDOPHYTA}

Lycopodium tristachyum Pursh-Orient Point, September 2, 19 Io.

\section{SPERMATOPHYTA}

Potamogeton foliosus Raf.-In a pond at Mattituck; determined at Bureau of Plant Industry, Washington. 
Potamogeton hybridus Sheld.-In a pond at Southold; determined at Bureau of Plant Industry.

Festuca Shortii Kunth-The specimen reported in the Third Supplementary list from wet woods at Mattituck; should be referred to Festuca nutans Spreng.

Glyceria canadensis (Mx.) Trin.-Swamp at Mattituck; determined by Mrs. Agnes Chase.

Panicum mattamuskeetense Ashe-Laurel; determined by Mrs. Chase.

Panicum Wrightianum Scribn.--Southold, the host of Phyllachora puncta. N. Y. State Mus. Bull. 243-244: 92. Issued May I5, 1923.

Setaria lutescens (Weigel) Hubbard-Sandy field at Laurel; determined by Mrs. Chase.

Carex Howei Mackenzie-Greenport in low wet woods; determined at N. Y. Botanical Garden.

Carex retroflexa Muhl.-Gardiner's Island in dry woods; determined at N. Y. Botanical Garden.

Xyris caroliniana Walt.-Sandy swamp at Laurel, August I7, 1923.

Spiranthes vernalis Engelm. \& Gray-Peconic in low grassy fields; determined at N. Y. Botanical Garden.

Juglans nigra L.-One large tree in woods at Mattituck.

Chenopodium murale L.-Orient in waste ground; determined at N. Y. Botanical Garden.

A maranthus caudatus L.-Occasional as a weed in cultivated and waste grounds at Orient; determined at. N. Y. Botanical Garden.

Spergularia canadensis (Pers.) Don-"Alsine canadensis (Pers.) House; (Arenaria canadensis Pers.; Spergularia borealis Robinson)-A species of the northern shores of eastern America, which appears to reach its southern limit of distribution on Shelter Island, opposite Greenport, where collected by Dr. C. H. Peck in $187 \mathrm{I}$ (State Herbarium)." N. Y. State Mus. Bull. 243-244: 57. Issued May I5, I923.

Nymphozanthus advena (Ait.) Fernald-Swamp at Laurel, August I6, I923.

Ranunculus laxicaulis (T. \& G.) Darby-Mattituck in a swamp, September I, I923.

Arabis lyrata L.-Cultivated fields at Orient; determined at Bureau of Plant Industry.

Potentilla recta L.-Sandy fields at Laurel, July I6, I923: and Orient, June 20, 1923.

Linum medium (Planch.) Britton-Dry woods at Southold; determined at Bureau of Plant Industry.

Myriophyllum tenellum Bigel.-Sandy shore of a large pond at Southold, August 20, 1923.

Cornus Amomum L.- "Border of swamp near Orient. Roy Latham, September 2." N. Y. State Mus. Bull. 243-244: 57. Issued May I5, 1923 .

Nymphoides lacunosum (Vent.) Fernald - In a pond at Southold, August 20, 1923.

Echium vuıgare L.-Laurel in old pastures; determined at N. Y. Botanical Garden. 
Mentha citrina Ehrh.-Greenport in waste places; determined at N. Y. Botanical Garden.

Limosella aquatica L., var. tenuifolia (Wolf.) Pers.-Fishers Island, no. 743. September 15, I891. Rev. J. L. Zabriskie.

Jasione montana L.-Sandy fields and roadsides at Laurel, August 17, 1923; determined at Bureau of Plant Industry.

Artemisia Absinthium L.-Roadsides and waste places at Greenport; determined at N. Y. Botanical Garden.

Coreopsis rosea Nutt.-Sandy swamp at Laurel, August I6, I923.

Eupatorium album L.-Sandy fields at Laurel; determined at Bureau of Plant Industry.

Heliopsis helianthoides (L.) Sweet-Rich open woods at Mattituck, July 3, 1923. The plants not quite typical, some of the plants having leaves little rougher than usual.

Lactuca canadensis L., var. montana Britton-Cutchogue and Orient. Reported in the Second Supplementary list as Lactuca canadensis, var. integrifolia (Bigel.) Gray; which included Lactuca canadensis, var. montana Britton and Lactuca sagittifolia Ell. of the Preliminary list. A revised description of this variety is given by Dr. H. D. House, in N. Y. State Mus. Bull. 243-244: 58. Issued May 15, I923. "Leaves thin, acuminate, pale beneath, tapering to a sessile sagittate-clasping base, the lower ones sparingly sinuate-toothed toward the base, the upper ones all entire; stem glabrous and glaucous; achenes black, oval, flattened, one-nerved on each face, finely pitted and transversely wrinkled, the beak about equal to the body of the achene in length; pappus white; flowers apparently yellow.

"This is doubtless the plant reported by Burnham and Latham (Torreya 14:252. 1914) as L. sagittifolia Ell, which is not uncommon in southern New York. The plants here described were collected by Roy Latham, at Cutchogue, town of Southold, and presented to the state herbarium."

Xanthium italicum Mor.-Locally common in cultivated and waste grounds at Greenport; determined at N. Y. Botanical Garden.

This supplementary list brings the total number of species reported by the authors from the town of Southold to:-Insect Galls, 95; Slime-molds, 20; Algae, 102; Fungi, 928; Lichens, I27; Hepatics, 32; Mosses, III; Ferns, 37; Flowering Plants, I 160 . 
Reprinted from TORREYA, Vol, 24, March-April, I924.

\title{
THE FLORA OF THE TOWN OF SOUTHOLD, LONG ISLAND AND GARDINER'S ISLAND, NEW YORK
}

\author{
Stewart H. Burnham and Roy A. Latham
}

Fourth Supplementary List*

INSECT GALLS

Neuroterus noxiosus Bass.-Leaves of Quercus Prinus at Greenport; determined by Dr. M. D. Leonard.

Phyllocoptes quadripes Shim.-Leaves of Acer rubrum at Greenport; determined by Dr. Leonard.

\section{MYXOMYCETES}

Badhamia rubiginosa (Chev.) Rost.-Old leaves; determined by Prof. John Dearness.

Clathroptychium rugulosum (Wallr.) Rost.-Orient on bark of Juniperus virginiana; determined by Prof. Dearness.

Cribraria rufa (Roth) Rost.-Old wood at Southold; determined by Prof. Dearness.

Hemitrichia vesparium (Batsch) Macbr.-Greenport; determined by Prof. Dearness.

Stemonitis splendens Rost.-Rich woods on rotten wood at Greenport; determined by Prof. Dearness.

\section{EUPHYCEAE}

Lithothamnion polymorphum (L.) Aresch.-On stones in the Sound at Orient; determined by Dr. R. W. Miner.

\section{PHYCOMYCETES}

Aspergillus candidus Link-Orient on dead plants; determined by Prof. Dearness.

Peronospora Arthurii Farl.-On Oenothera, Southold; determined by Prof. Dearness.

\section{ASCOMYCETES (excluding PYRENOMYCETES)}

Aleuria repanda (Pers.) Gill.--Inside woodpecker's hole of an apple tree at Greenport; determined by Prof. Dearness.

Ascobolus Crouani Boud.-Old stems of Brussels Sprouts, Brassica oleracea gemmifera at Orient; determined by Prof. Dearness.

* The Preliminary flora was published in Torreya 14: 20I-225, Nov. I9I4 and 229-254, Dec. I9I4. The First Supplementary list was published in Torreya 17: III-I22, July I9I7. The Second Supplementary list was published in Torreya 21: I-I I, Jan.-Feb. I92I and 28-33, March-April I92I. The Third Supplementary list was published in Torreya 23: 3-9, Jan,-Feb. I 923 and 25-3I, March-April I923. 
581.974 .721
2 